Aus der Klinik für Anästhesiologie

(Prof. Dr. med. K. Meissner)

der Medizinischen Fakultät der Universität Göttingen

\title{
Der PEEP und seine Auswirkung auf die mechanische Leistung bei der maschinellen Beatmung
}

\author{
INAUGURAL-DISSERTATION \\ zur Erlangung des Doktorgrades \\ der Medizinischen Fakultät der \\ Georg-August-Universität zu Göttingen
}

vorgelegt von

Tim Behnemann

aus

Buxtehude

Göttingen 2019 
Dekan:

Betreuungsausschuss

Betreuer/in

Ko-Betreuer/in:

\section{Prüfungskommission}

Referent/in

Ko-Referent/in:

Datum der mündlichen Prüfung:
Prof. Dr. med. W. Brück

Prof. Dr. med. O. Mörer

Prof. Dr. med. S. Andreas

Prof. Dr. med. O. Mörer

Prof. Dr. med. S. Andreas 
Hiermit erkläre ich, die Dissertation mit dem Titel "Der PEEP und seine Auswirkung auf die mechanische Leistung bei der maschinellen Beatmung" eigenständig angefertigt und keine anderen als die von mir angegebenen Quellen und Hilfsmittel verwendet zu haben.

Göttingen, den 
Die Daten, auf denen die vorliegende Arbeit basiert, wurden teilweise publiziert:

Collino F, Rapetti F, Vasques F, Maiolo G, Tonetti T, Romitti F, Niewenhuys J, Behnemann T, Camporota L, Hahn G et al. (2019): Positive End-expiratory Pressure and Mechanical Power. Anesthesiology 130, 119-130 


\section{Inhaltsverzeichnis}

Abbildungsverzeichnis ................................................................................. III

Tabellenverzeichnis ......................................................................................... III

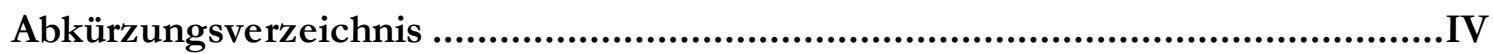

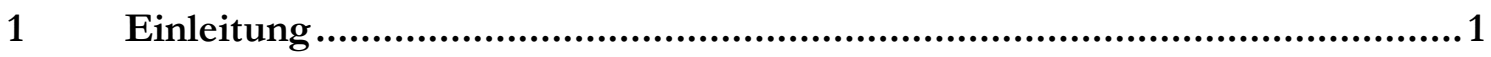

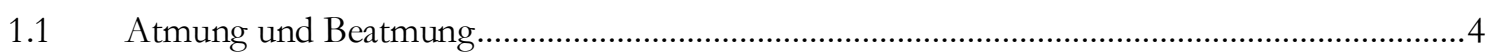

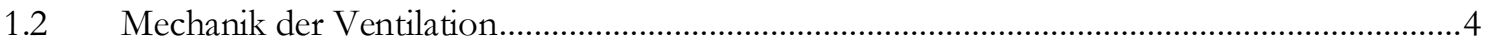

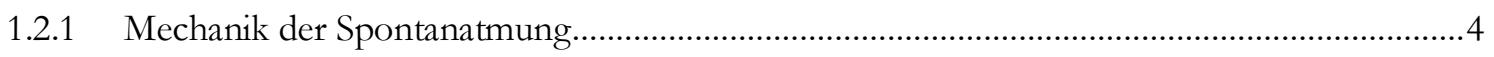

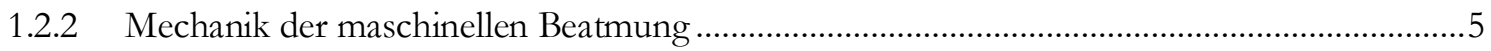

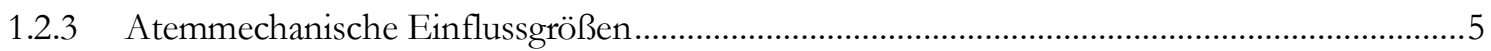

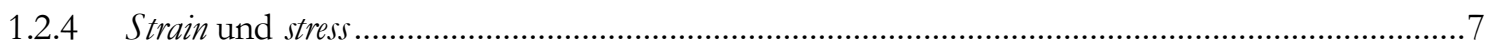

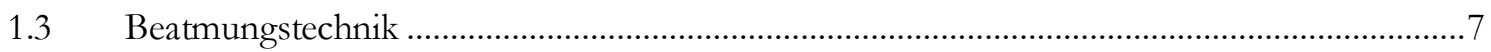

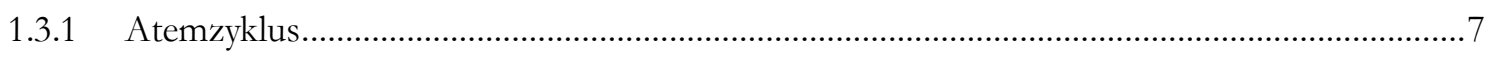

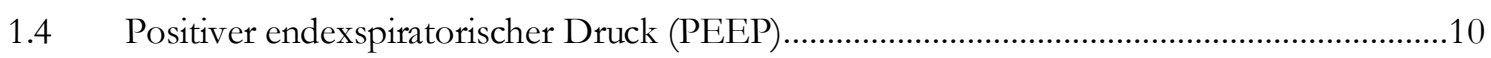

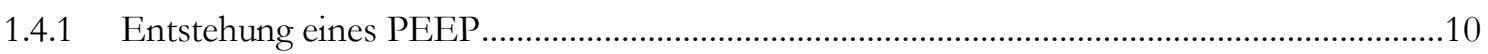

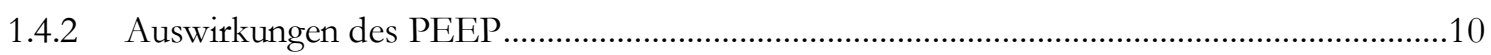

$1.5 \quad$ Nebenwirkungen der maschinellen Beatmung.......................................................................14

1.5.1 Nebenwirkungen auf Grund des veränderten Pleuradrucks..................................................15

1.5.2 Nebenwirkungen auf Grund des veränderten transpulmonalen Drucks................................16

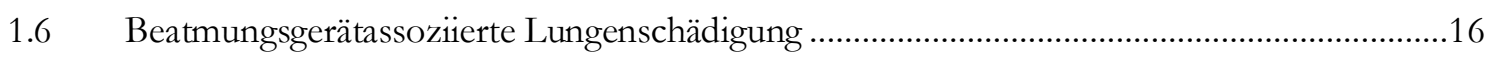

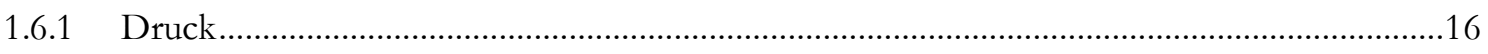

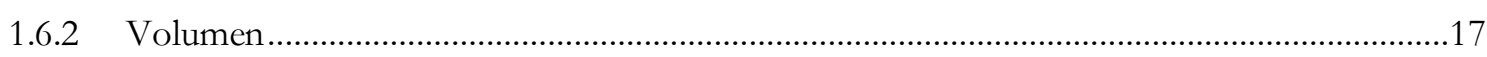

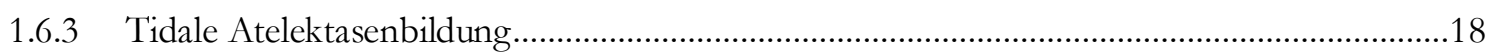

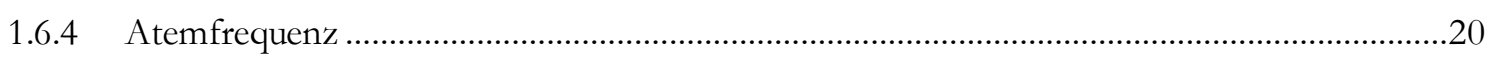

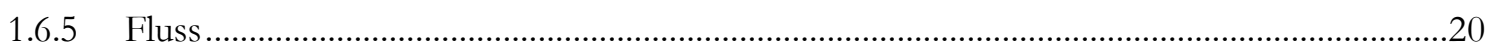

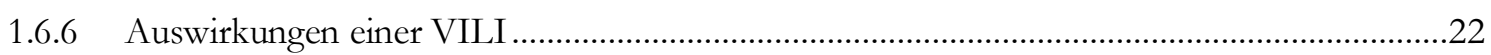

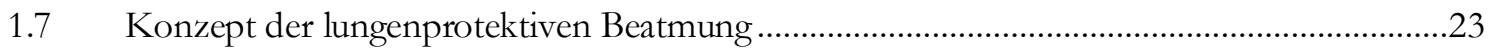

1.8 Die mechanische Leistung der maschinellen Beatmung ..........................................................24

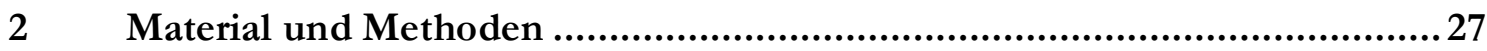

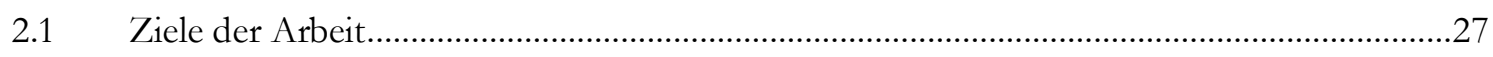

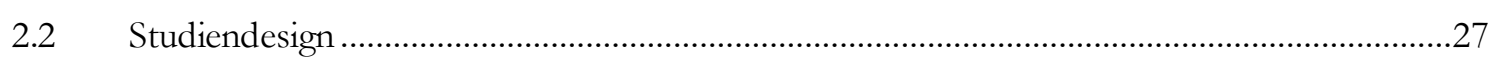

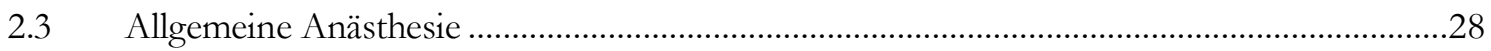

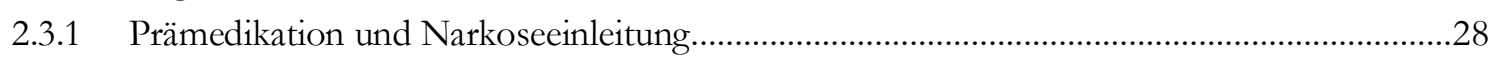

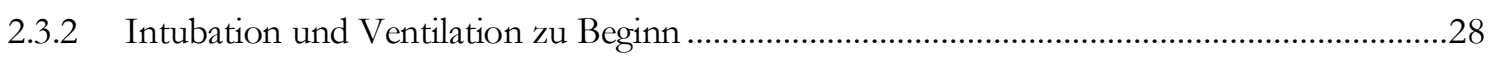

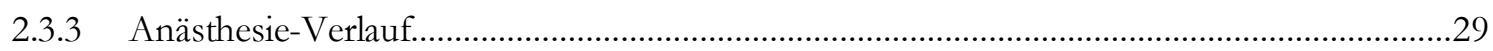

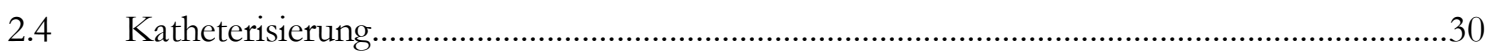

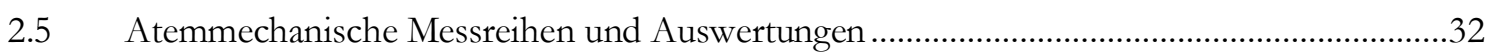




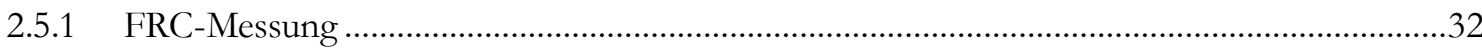

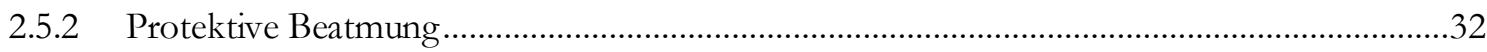

2.5.3 Baseline-Messung und Messreihen.......................................................................................33

2.6 Übersicht der erhobenen und berechneten Parameter.........................................................35

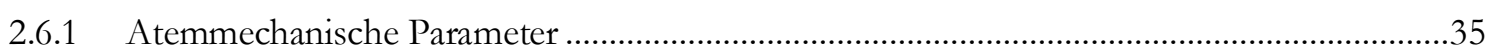

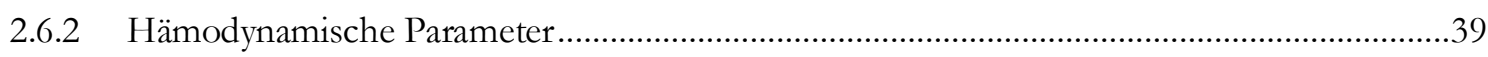

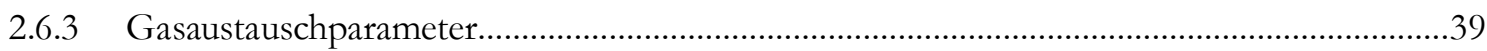

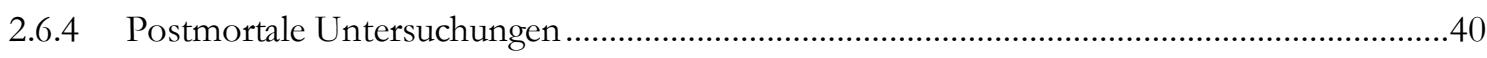

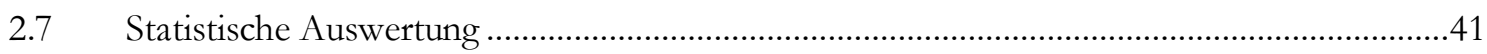

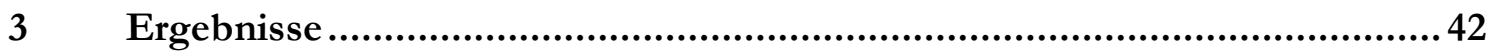

3.1 Allgemeine Ergebnisse zu den Versuchstieren ............................................................42

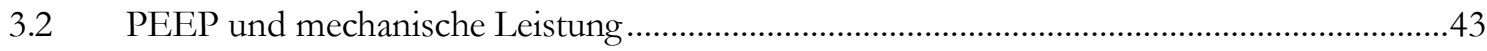

3.3 Gewählter PEEP und mechanische Leistung im zeitlichen Verlauf ..................................45

3.4 Gewählter PEEP in Bezug zu den lungenmechanischen Eigenschaften..............................48

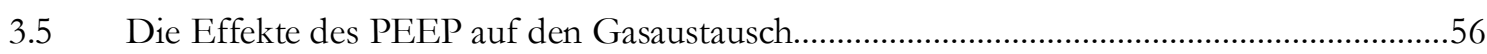

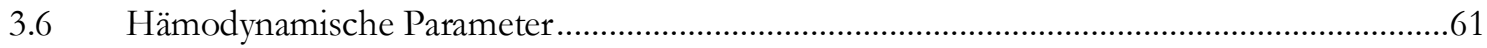

3.7 PEEP und seine Auswirkungen auf die Lungenstruktur.................................................67

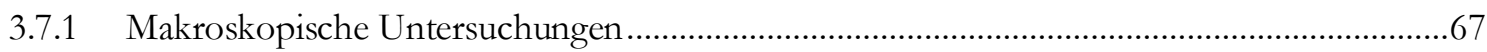

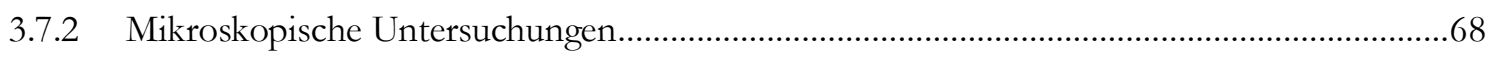

3.7.3 Lungenödem: Lungengewicht und $W e t-t o-d y$-Ratio..........................................................73

3.8 PEEP und die Grenzwerte der mechanischen Leistung .................................................74

$4 \quad$ Diskussion ..................................................................................... 76

4.1 Der Einfluss des PEEP auf die mechanische Leistung...................................................77

4.2 Mechanische Leistung und PEEP im zeitlichen Verlauf.....................................................79

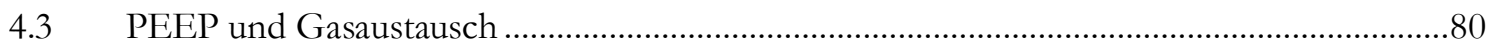

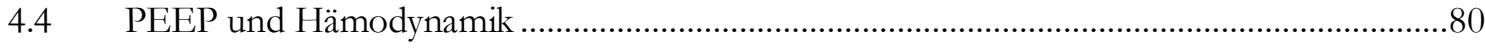

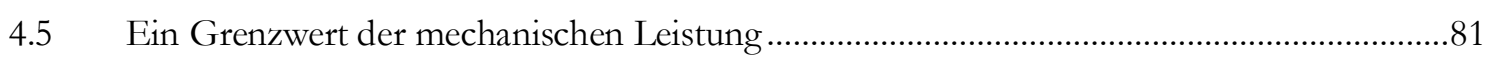

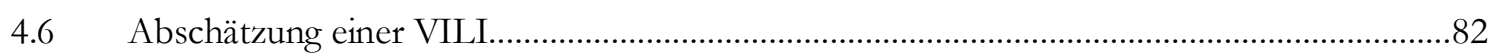

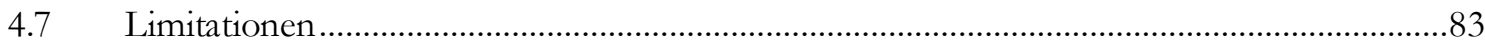

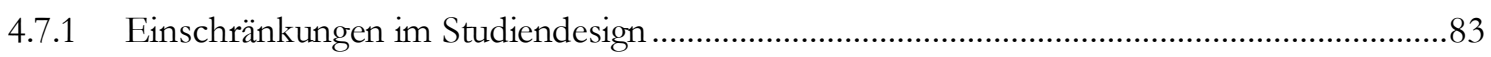

4.7.2 Einschränkungen des Konzepts der mechanischen Leistung............................................84

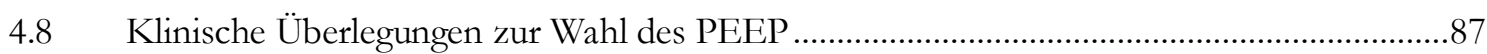

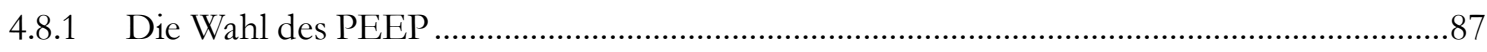

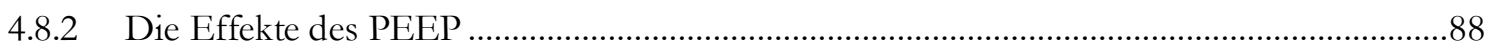

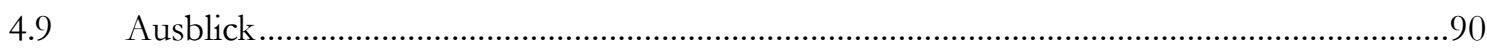

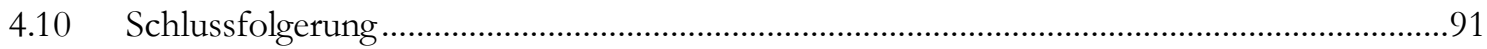

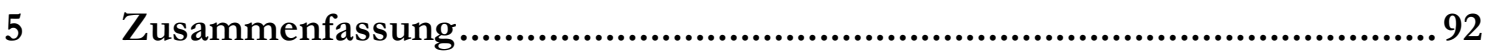

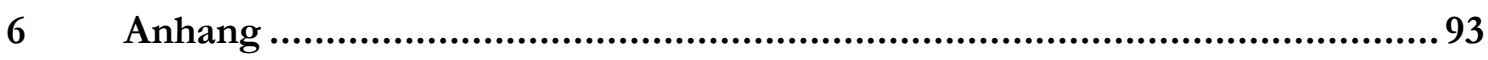

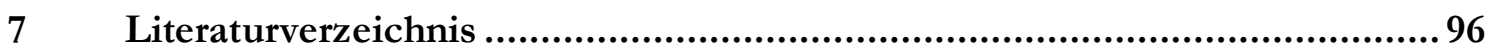




\section{Abbildungsverzeichnis}

Abbildung 1: Ruhedehnungskurve der Lunge

Abbildung 2: Phasen des Beatmungszyklus bei volumenkontrollierter Beatmung ohne PEEP (links) und mit PEEP (rechts)

Abbildung 3: Ablauf der Katheterisierung und Versuchsaufbau..................................................

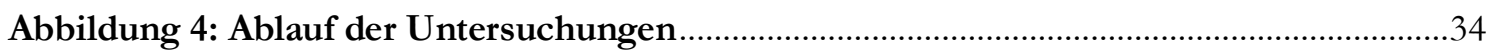

Abbildung 5: Mechanische Leistung: Lunge (A) und respiratorisches System (B) in

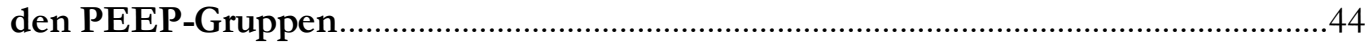

Abbildung 6: Mechanische Leistung und Arbeit im zeitlichen Verlauf......................................46

Abbildung 7: Überlebende Versuchstiere im Verlauf der Zeit (Kaplan-Meyer-Kurve) ...........47

Abbildung 8: Respiratorische Parameter im zeitlichen Verlauf..............................................49

Abbildung 9: Veränderungen der Elastance-Werte im zeitlichen Verlauf ..................................51

Abbildung 10: Stress, totaler strain und spezifische Lungenelastance im zeitlichen

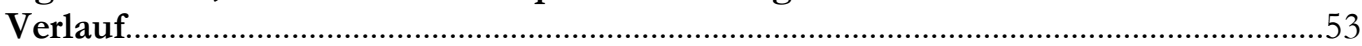

Abbildung 11: Parameter des Gasaustauschs im zeitlichen Verlauf ...........................................57

Abbildung 12: Parameter des Säure-Base-Haushalts im zeitlichen Verlauf ..............................58

Abbildung 13: Hämodynamische Parameter des Körperkreislaufes im zeitlichen

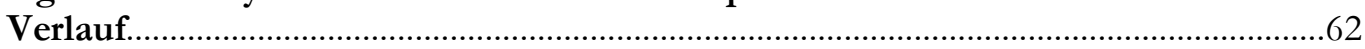

Abbildung 14: Gefäßdrücke im pulmonalen Kreislauf im zeitlichen Verlauf ...........................63

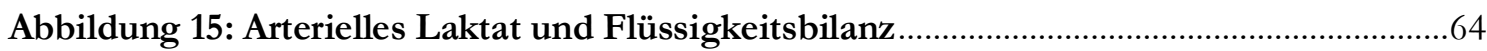

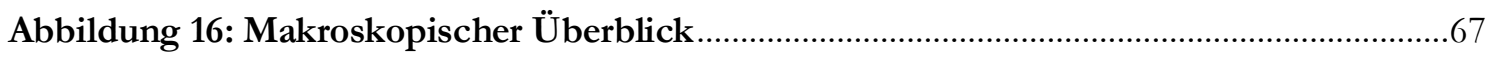

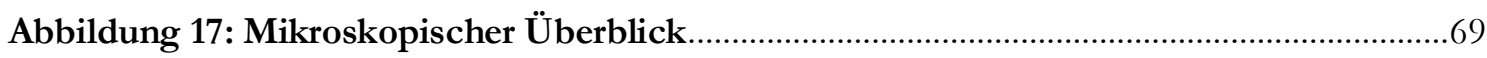

Abbildung 18: Verteilung der häufigsten mikroskopischen Läsionen nach PEEPGruppe …….....................................................................................................

Abbildung 19: Verteilung der selteneren mikroskopischen Läsionen nach PEEP-

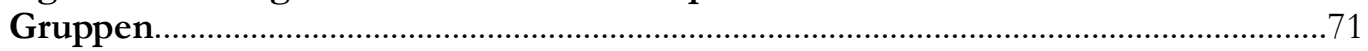

Abbildung 20: Wet-to-dry-Ratio-Index in den verschiedenen Organsystemen .........................73

\section{Tabellenverzeichnis}

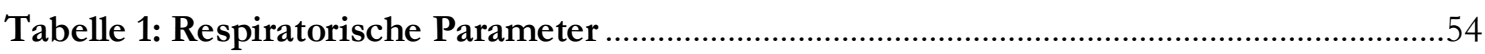

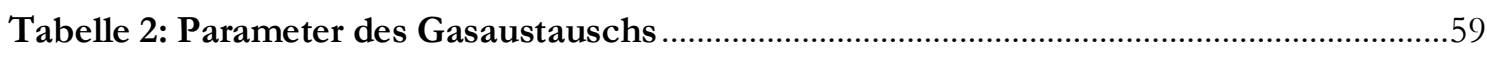

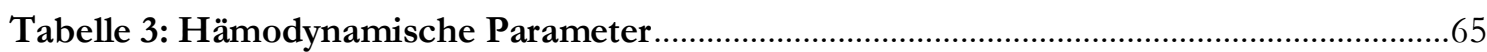

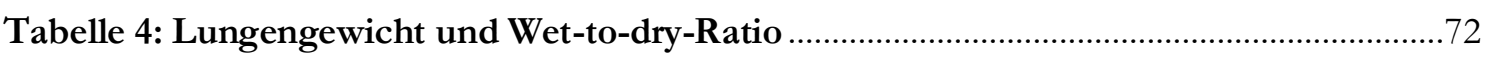

Tabelle 5: Mögliche Grenzwerte der mechanischen Leistung ................................................ 75 


\section{Abkürzungsverzeichnis}

Eine Übersicht für die in den Formeln verwendeten Symbolbezeichnungen befindet sich im Anhang.

\begin{tabular}{|c|c|}
\hline ANOVA & analysis of variance \\
\hline A. & Arteria \\
\hline $\mathrm{AF}$ & Atemfrequenz \\
\hline ARDS & acute respiratory distress syndrome (akutes Lungenversagen) \\
\hline AUC & area under the curve (Fläche unter der Kurve/Integral) \\
\hline $\mathrm{BE}$ & baseexcess (Basenüberschuss) \\
\hline BGA & Blutgasanalyse \\
\hline bpm & beats per minute (Schläge pro Minute) \\
\hline $\mathrm{C}$ & Compliance \\
\hline CI & confidence interval (Vertrauensintervall) \\
\hline $\mathrm{cmH}_{2} \mathrm{O}$ & Zentimeter Wassersäule \\
\hline CMV & controlled mechanical ventilation (kontrollierten Beatmung) \\
\hline $\mathrm{CT}$ & Computer-Tomographie \\
\hline EELV & End-exspiratorisches Volumen \\
\hline EIT & Elektrische Impedanztomografie \\
\hline EKG & Elektrokardiogramm \\
\hline E & Elastance \\
\hline etCO ${ }_{2}$ & Endtiales Kohlenstoffdioxid \\
\hline EVLW & Extravasales Lungenwasser \\
\hline $\mathrm{F}$ & Atemgasfluss \\
\hline $\mathrm{FiO}_{2}$ & Inspiratorische Sauerstofffraktion \\
\hline FRC & Funktionelle Residualkapazität \\
\hline G40\% & Glukose $40 \%$ \\
\hline $\mathrm{Hb}_{\mathrm{O}_{2}}$ & Sauerstoffsättigung des Hämoglobins \\
\hline H1 & Tatsächliche Experimentdauer des individuellen Schweins \\
\hline $\mathrm{Hb}$ & Hämoglobin \\
\hline $\mathrm{HE}$ & Hämatoxylin-Eosin \\
\hline $\mathrm{HZV}$ & Herzzeitvolumen \\
\hline I:E-Verhältnis & Atemzeitverhältnis \\
\hline I.E. & Internationale Einheiten \\
\hline i.m. & Intramuskulär \\
\hline $\mathrm{mEq}$ & Milliäquivalent \\
\hline $\mathrm{mmHg}$ & Millimeter Quecksilbersäule \\
\hline $\mathrm{P}$ & Druck \\
\hline $\mathrm{PaCO}_{2}$ & Arterieller Kohlenstoffdioxidpartialdruck \\
\hline $\mathrm{PaO}_{2}$ & Arterieller Sauerstoffpartialdruck \\
\hline
\end{tabular}


$\mathrm{PaO}_{2} \quad$ Sauerstoffpartialdruck

PAP pulmonary artery pressure (Pulmonalarteriendruck)

PBW per ideal (body)weight (per idealem (vorhergesagtem) Körpergewicht)

PCV pressure-controlled ventilation (druckkontrollierte Beatmung)

PCWP pulmonary capillary wedge pressure (Lungenkapillaren-Verschlussdruck)

PEEP Positiver endexspiratorischer Druck

$\mathrm{PiCCO}$ pulse contour cardiac output (Pulskontur-Herzzeitvolumen)

$\mathrm{R}$ Widerstand

R-L-Shunt Rechts-Links-Shunt resp. Respiratorisch

ROC receiver operating characteristic

$\mathrm{SpO}_{2}$ Periphere Sauerstoff-Sättigung

TLC total lung capacity (totale Lungenkapazität)

$\mathrm{V}$

Volumen

$\mathrm{V}$. vena volume-controlled ventilation (volumenkontrollierte Beatmung)

VILI ventilator-induced lung injury

W/D-Index Wet-to-dry-Index

W/D-Ratio Wet-to-dry-Ratio

ZEEP zero PEEP (kein applizierter positiver endexspiratorischer Druck) Zentralvenöser Druck 


\section{$1 \quad$ Einleitung}

Die maschinelle Beatmung stellt heutzutage eine bedeutende Therapiemaßnahme in der Intensivmedizin bei der Behandlung der akuten respiratorischen Insuffizienz dar, denn sie gewährleistet eine teilweise oder komplette Übernahme der lebenswichtigen Atemaktivität durch ein Beatmungsgerät (Gattinoni et al. 2017b). Das Hauptziel ist dabei die Gewährleistung eines suffizienten Gasaustauschs in dem Zeitintervall, das notwendig ist, die zugrundeliegende Ursache für die Beatmungsindikation zu beheben (DGAI 2017). Um diesen Gasaustausch sicherzustellen, muss die Lunge beatmet werden, indem während der Inspiration das Beatmungsgerät eine Druckdifferenz erzeugt und dadurch ein bestimmtes Volumen in die Lunge befördert (Pape et al. 2018). Die dabei durch die (transpulmonale) Druckdifferenz auftretende mechanische Spannung im Lungengewebe wird als stress bezeichnet (Protti et al. 2011). Das dabei applizierte Tidalvolumen führt zu einer Aufweitung des Alveolarraums, welches eine als strain beschriebene Dehnungsbelastung der Lunge hervorruft (Verhältnis des Tidalvolumen zur funktionellen Residualkapazität (FRC)) (Protti et al. 2011). Während der Exspiration wird dieses Volumen auf Grund passiver Rückstellkräfte wiederum ausgeatmet, indem die zuvor aufgebaute Druckdifferenz auf den Atmosphärendruck oder einen zuvor eingestellten positiven endexspiratorischen Druck (PEEP) abfällt (Lumb und Nunn 2010). Ein PEEP ist ein bestimmtes minimales Druckniveau, das während des gesamten Atemzyklus auf die Alveolen wirkt (Lumb und Nunn 2010).

Wie jede Therapieform birgt auch die maschinelle Beatmung Nebenwirkungen. Zu den pulmonalen Nebenwirkungen zählt die beatmungsinduzierte Lungenschädigung (ventilator induced lung injury, VILI), welche sich durch eine mechanische Schädigung der Alveolarstruktur mit meist anschließender Entzündungsreaktion auszeichnet (Parker et al. 1993; Tonetti et al. 2017). Die Ursache eines dabei auftretenden Schadens liegt in der Interaktion zwischen Beatmungsgerät und Lunge und dementsprechend ist das Ausmaß des Schadens sowohl von den Einstellungen des Beatmungsgerätes als auch von den atemmechanischen Eigenschaften der (möglicherweise vorgeschädigten) Lunge abhängig (Gattinoni et al. 2017a).

In Bezug auf mögliche beatmungsgerätbezogene Ursachen für die mechanische Alveolarschädigung im Rahmen der VILI wurden in den letzten Jahren viele Faktoren untersucht. Pathophysiologisch wurden so zunächst das Barotrauma auf Grund zu hoher 
Beatmungsdrücke (Kumar et al. 1973; Oczenski et al. 2012) und das Volutrauma auf Grund eines zu hohen Beatmungsvolumens (Dreyfuss et al. 1988) beschrieben. Außerdem konnte gezeigt werden, dass das zyklische Öffnen und Kollabieren von Alveolen zu einem Atelektrauma führen kann (Tremblay et al. 1997). Für andere Beatmungsparameter wie die Atemfrequenz und den inspiratorischen Fluss gibt es - weitestgehend isoliert betrachtet - auch Hinweise auf eine schädliche Wirkung außerhalb physiologischer Grenzen (Protti et al. 2016).

Auf Grund dieser den Beatmungsparametern zugeschriebenen Schäden wurde das Konzept der lungenprotektiven Beatmung erstellt, dessen Ziel es ist, sämtliche Beatmungsparameter in einem als sicher geltenden Rahmen zu halten, sodass die oben genannten Schäden reduziert werden können (Tobin 2000). In diesem Zusammenhang gilt die Applikation eines PEEP vor allem im Zusammenhang mit der Lungenödembildung und der Ausbildung eines Atelektraumas als lungenprotektiv (Webb and Tierney 1974; Tremblay et al. 1997). Da allerdings die Aufrechterhaltung eines suffizienten Atemgasaustauschs das oberste Ziel der Beatmung darstellt, müssen beispielsweise bei Patienten, bei denen das Tidalvolumen zur Verhinderung eines drohenden Volutraumas nicht weiter erhöht werden sollte, andere Beatmungsparameter wie die Beatmungsfrequenz hochreguliert werden (DGAI 2017). In der Beatmungstherapie gibt es viele solcher Situationen, in denen die alleinige Betrachtung eines einzelnen Beatmungsparameters nicht das gesamte mögliche Schädigungspotenzial der maschinellen Beatmung erfassen kann, da zum Beispiel der angenommene potenziell schädliche Beitrag durch die Beatmungsfrequenz steigt. Dadurch stellt sich die Frage, welcher Beatmungsparameter den größten und entscheidenden Anteil zur Entstehung einer VILI beiträgt. Gibt es eine Priorisierung der Schädigungspotentiale einzelner Parameter oder sogar eine vereinheitlichende singuläre Größe?

Das mechanische Schadensausmaß der einzelnen Beatmungsparameter scheint sich durch die kontinuierlich applizierte Druck-Volumen-Arbeit auf das Lungenparenchym je nach dynamischen Ausmaß zu ergeben (Cressoni et al. 2016). Diese mechanische Arbeit in Bezug auf die Zeitkomponenten (zum Beispiel Atemfrequenz) wird durch die mechanische Leistung zusammengefasst und könnte als ganzheitliches Konzept in der pathophysiologischen Entwicklung einer VILI ein besseres Verständnis liefern (Gattinoni et al. 2016). Die Beatmungsparameter würden darin mithilfe einer Formel für die mechanische Leistung in einen mathematischen Zusammenhang gebracht werden können (Gattinoni et al. 2016). Dabei 
konnte gezeigt werden, dass ein Lungenschaden bei Schweinen erst ab einer gewissen Grenzwertüberschreitung der applizierten Leistung auftrat und in einem solchen Fall vom Ergotrauma gesprochen werden könnte (Cressoni et al. 2016; Marini and Jaber 2016). Ob ein Schaden vom Beatmungsgerät ausgeht, könnte folglich mithilfe der Formel als Summe der einzelnen potentiellen Noxen durch die verschiedenen Leistungskomponenten vorhergesagt werden (Gattinoni et al. 2016). Theoretisch müsste dabei ein applizierter PEEP als Teil des auf die Lunge wirkenden Drucks einen Anteil zur mechanischen Leistung beitragen (Gattinoni et al. 2016). Experimentell wurde dies bislang noch nicht bestätigt, sodass sich die Frage stellt, ob ein applizierter PEEP tatsächlich als Teil der verabreichten mechanischen Leistung zum beschriebenen Ergotrauma beiträgt und somit womöglich mehr schädliches Potenzial aufweist, als dem bisher als protektiv geltenden PEEP zugeschrieben worden ist.

In dieser Dissertation soll daher der Einfluss unterschiedlich hoher PEEP-Werte auf einen sich entwickelnden Lungenschaden tierexperimentell untersucht werden. Dazu werden 40 weibliche Schweine über einen Zeitraum von 50 Stunden maschinell beatmet, indem mithilfe der zuvor ermittelten FRC das Tidalvolumen so gewählt wird, dass sich für jedes Tier ein strain von 1,0 ergibt (Tidalvolumen $=$ FRC). Sämtliche andere Beatmungsparameter werden vergleichbar und konstant gehalten, sodass die sich lediglich im gewählten PEEP-Wert unterscheidenden Versuchstiere in sechs Gruppen mit PEEP-Werten 0, 4, 7, 11, 14 und 18 $\mathrm{cm} \mathrm{H}_{2} \mathrm{O}$ eingeteilt wurden. So sollte überprüft werden, ob die isolierte Erhöhung des PEEP ein Ergotrauma auslösen, verstärken oder anderweitig beeinflussen kann, um letztlich zu klären, ob der PEEP, wie weitläufig angenommen, protektiv oder schädlich ist. Dabei sollten pathologische Untersuchungen und die Entwicklung von Flüssigkeitsansammlungen in der Lunge als Marker für eine aufgetretene Beschädigung der Lunge herangeführt, aber auch kritisch beleuchtet werden. Weiterhin werden die atemmechanischen Parameter (wie die mechanische Leistung) analysiert, um einen möglichen Grenzwert der mechanischen Leistung für das Auftreten eines beatmungsgerätassoziierten Lungenschadens zu definieren, welcher den PEEP berücksichtigt. Bei der Patientenanwendung könnte mithilfe des Konzepts der mechanischen Leistung und deren Grenzwert ein sicheres Kriterium entwickelt werden, bis wann eine bestimmte Konstellation maschineller Beatmungsparameter unter Berücksichtigung individueller Lungeneigenschaften eine Lungenprotektion gewährleisten kann. 


\subsection{Atmung und Beatmung}

Atmung ist der lebensnotwendige Gasaustausch zwischen Organismus und Umwelt, damit ein Lebewesen mithilfe des aufgenommenen Sauerstoffs $\left(O_{2}\right)$ unter anderem Energie erzeugen und das Abfallprodukt Kohlenstoffdioxid $\left(\mathrm{CO}_{2}\right)$ abgeben kann (Oczenski et al. 2012; Pape et al. 2018). Die Atmung wird in die drei Teilprozesse Ventilation, Diffusion und Perfusion unterteilt (Pape et al. 2018). Dabei liegt der Hauptunterschied zwischen physiologischer Atmung und maschineller Beatmung in der Ventilation. In beiden Fällen ergibt sich die Belüftung der Alveolen durch eine periodische Veränderung der intrathorakalen Druckverhältnisse (Lumb und Nunn 2010).

\subsection{Mechanik der Ventilation}

\subsubsection{Mechanik der Spontanatmung}

Bei der Spontanatmung führt während der Inspiration die Kontraktion der Inspirationsmuskeln (v.a. der posteriore, muskuläre Anteil des Zwerchfells) dazu, dass sich der Thorax ausdehnt (DGAI 2017). Auf Grund der Kopplung der Pleuren durch die kapillare Adhärenz folgt die Lunge diesen Bewegungen des Thorax und nimmt ebenfalls an Volumen zu. Nach dem Boyle-Mariotte-Gasgesetz, eine Folgerung des idealen Gasgesetzes, folgt, dass das Produkt aus Druck $(P)$ und Volumen $(V)$ näherungsweise konstant ist (Pape et al. 2018). Daraus ergibt sich bei einer Zunahme des Thoraxvolumens eine Abnahme des intrathorakalen Drucks ( $=$ intrapleuraler Druck $\left.\left(P_{\text {pleu }}\right)\right)$. Dadurch erhöht sich der transpulmonale Druck (Druckdifferenz zwischen dem intraalveolären Druck und dem intrapleuralen Druck), wodurch sich die Lunge weiter ausdehnt und nach dem Boyle-Mariotte-Gesetz sich daher der intraalveoläre Druck verringert. Es entsteht daher ein Druckgradient zwischen Alveolarraum und dem Atmosphärendruck, welcher als treibende Druckdifferenz beim Atemgastransport fungiert. Durch diese Druckdifferenz wird entsprechend dem Ohm'schen Gesetz ein Atemzugvolumen (Tidalvolumen) durch Überwindung eines Strömungswiderstands (Resistance) in die Alveolen befördert (Lumb und Nunn 2010). Bei der Exspiration verkleinern die Retraktionskräfte von Lunge und Thorax und die Oberflächenspannung in den Alveolen das Lungenvolumen, wodurch der intraalveoläre Druck ansteigt und der entstehende Druckgradient für einen Teilchenstrom aus der Lunge sorgt (Pape et al. 2018). Die bei der Spontanatmung verrichtete Atemarbeit durch die Atemmuskulatur beträgt bei einem gesunden

Erwachsenen etwa $0,7-1,0 \frac{J}{l}$ (Rathgeber 2010). 


\subsubsection{Mechanik der maschinellen Beatmung}

Die maschinelle Beatmung unterstützt oder übernimmt letztlich die Funktion der Atemmuskulatur, welche aus vielen möglichen Gründen nicht vollständig den Anforderungen einer adäquaten Ventilation und damit einer Oxygenierung genügt (Gattinoni et al. 2017b). Die maschinelle Beatmung ist daher definiert als Maßnahme zur Erhaltung des Atemminutenvolumens durch externe Kräfte (Lumb und Nunn 2010). Eine künstliche Beatmung und die damit verbundene Aufblähung der Lunge durch einen Anstieg des transpulmonalen Drucks kann (abgesehen von Mischformen) in zwei gegensätzlichen Möglichkeiten erfolgen: Entweder wird eine Überdruckbeatmung oder eine Unterdruckbeatmung gewählt. Wobei in beiden Fällen die transpulmonalen Druckdifferenzen zwischen Beatmungsgerät und Alveolarraum vergrößert werden, da bei der selten verwendeten Unterdruckbeatmung analog zur Spontanatmung der Druck im Alveolarraum verringert und bei der gebräuchlichen Überdruckbeatmung ein hoher Druck vom Beatmungsgerät erzeugt wird (Gattinoni et al. 2017b). Die Luftteilchen strömen diesen Druckgradienten nach und es kommt so zu einer Aufblähung der Lunge (Pape et al. 2018). Sowohl bei der Spontanatmung als auch bei der maschinellen Beatmung verändert sich der intrapleurale Druck. Bei der spontanen Atmung und der maschinellen Unterdruckbeatmung wird er negativer, während bei der maschinellen Überdruckbeatmung dieser Druck ansteigt (Gattinoni et al. 2017b). Die Exspiration ist während der Spontanatmung, aber auch während der Beatmung ein passiver Vorgang auf Grund der elastischen Retraktionskräfte von Lunge und Thorax (Lumb und Nunn 2010). Im Nachfolgenden wird bei Betrachtung der maschinellen Beatmung von der gebräuchlicheren Überdruckbeatmung ausgegangen.

\subsubsection{Atemmechanische Einflussgrößen}

Die intrinsischen Lungenelastitzitätseigenschaften werden durch die Compliance und Elastance beschrieben. Dabei ist die Compliance $(C)$ ein Maß für die Volumen-Dehnbarkeit der Lunge und beschreibt die elastischen Eigenschaften in Abhängigkeit vom jeweiligen Füllungs- und damit Dehnungszustand der Alveolen. Sie ist definiert als Verhältnis von Volumenänderung $(\Delta V)$ und der dazugehörigen Druckänderung $(\Delta p)$ (Lumb und Nunn 2010):

$$
C=\frac{\Delta V}{\Delta p}
$$

In einem Druck-Volumen-Diagramm (Ruhedehnungskurve) des respiratorischen Systems wird die Compliance als Steigung dargestellt. Auf Grund der dehnungsabhängigen Compliance-Werte ergibt sich ein sigmoidaler Verlauf, welche von zwei Umschlagspunkten, dem 
unteren und oberen Inflektionspunkt, in drei Abschnitte unterteilt wird (s. Abb. 1) (Pape et al. 2018). Im flachen unterem Kurvenabschnitt steigt die Compliance zunehmend stark an, ehe ab dem unteren Inflektionspunkt bis zum oberen Inflektionspunkt die Compliance linear zum applizierten Druck ansteigt. Dieser mittlere lineare Abschnitt entspricht dem Bereich um die normale FRC, wobei hier die größte Compliance erreicht wird. Auf Grund des besten Dehnungsverhaltens der Lungen fällt hier die Atemarbeit am geringsten aus. Im oberen flachen Kurvenabschnitt zeigt sich die maximale Alveolardehnbarkeit, da eine weitere Druckzunahme nur zu einer kleinen Volumenzunahme führt. Hierbei besteht die Gefahr die Alveolen durch eine Überblähung strukturell zu beschädigen. Der Reziprokwert der jeweiligen Compliance wird Elastance $(E)$ genannt und beschreibt die Steifigkeit der Lunge (Oczenski et al. 2012). Wird die Elastance in Beziehung zur jeweiligen FRC gesetzt, spricht man von der spezifischen Lungenelastanz $\left(E_{L_{\text {spec }}}\right)$ (Blankman et al. 2016).

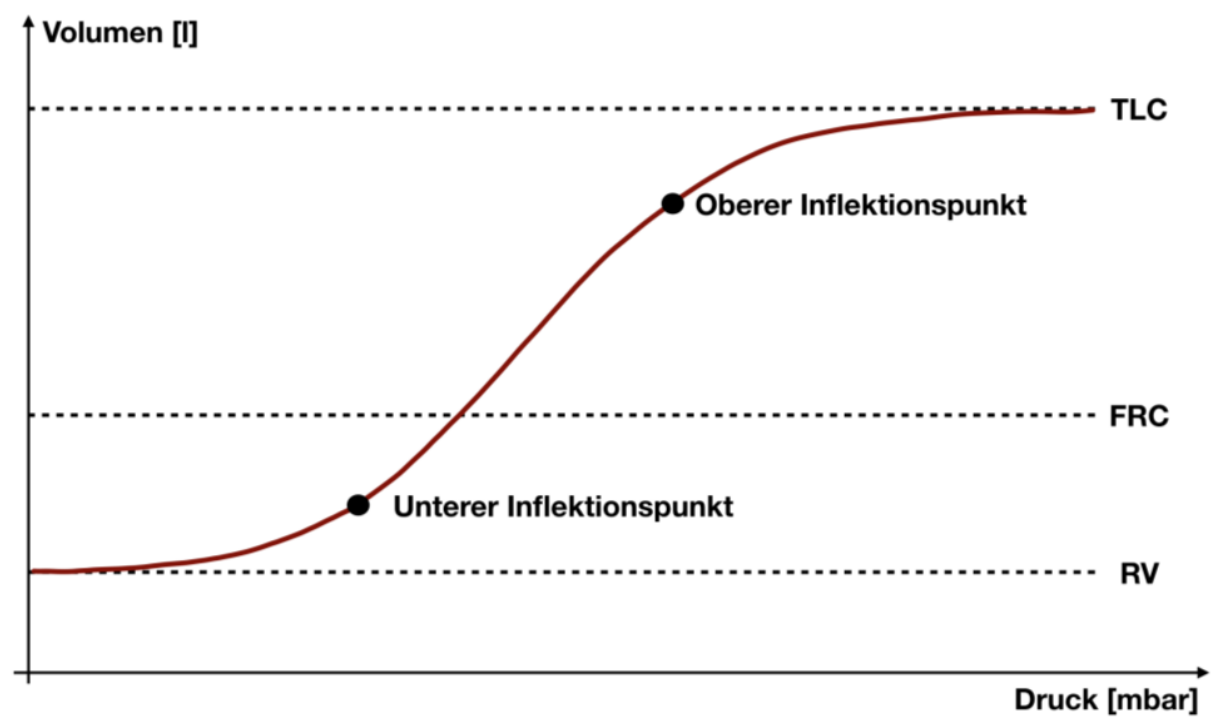

\section{Abbildung 1: Ruhedehnungskurve der Lunge}

Die Abbildung zeigt die Volumen-Druck-Beziehung der Lunge im Bereich zwischen dem Residualvolumen (RV) und der totalen Lungenkapazität (TLC). Bei zunehmendem Druck wird zunächst der untere und dann der obere Inflektionspunkt erreicht.

Die Resistance $\left(R_{a w}\right)$ ist ein Maß für den Strömungswiderstand (Atemwegswiderstand) des respiratorischen Systems und bestimmt daher den Luftstrom während der Inspiration und Exspiration. Die Resistance wird als Quotient aus treibender Druckdifferenz zwischen Atmosphäre/Beatmungsgerät und Alveolen $(\Delta p)$ sowie dem dabei auftretenden Atemgasfluss (F) definiert (Lumb und Nunn 2010):

$$
R_{a w}=\frac{\Delta p}{F}
$$




\subsubsection{Strain und stress}

Während der Ventilation der Lunge wirken physikalische Kräfte auf das Lungenparenchym. Die dabei auftretende mechanische Spannung (stress) ist physikalisch gesehen die Kraft pro Flächeneinheit, die auf einen Körper einwirkt. Dies entspricht dem Druck auf das Lungenfasergerüst, welcher dem während der Ventilation auf die Alveolen einwirkenden transpulmonalen Druck entspricht (Protti et al. 2013):

$$
\text { stress }=\text { Transpulmonaler Druck }
$$

Dahingegen beschreibt die Dehnung (strain) das Deformationsverhalten eines Körpers. Dies entspricht der Elongation der Lungenstrukturen durch das applizierte Tidalvolumen in Relation zur funktionellen Residualkapazität während der Ventilation (Protti et al. 2013):

$$
\text { strain }=\frac{\text { Tidalvolumen }}{\text { Funktionelle Residualkapazität }}
$$

\subsection{Beatmungstechnik}

Wird ein Teil der aufzubringenden Atemarbeit maschinell oder eine andere Person übernommen, wird von einer assistierten Beatmung gesprochen. Eine gänzliche Übernahme durch ein Beatmungsgerät oder eine beatmende Person wird kontrollierte Beatmung genannt (DGAI 2017).

\subsubsection{Atemzyklus}

Ein Atemzyklus entspricht der Zeitspanne von Beginn der Inspiration bis zum Abschluss der Exspiration und dessen Dauer $(\mathrm{T})$ setzt sich aus Inspirationszeit $\left(\mathrm{T}_{\mathrm{Insp}}\right)$ und Exspirationszeit $\left(\mathrm{T}_{\mathrm{Exp}}\right)$ zusammen. Das Atemzeitverhältnis (I:E-Verhältnis) ist der Quotient aus Inspirations- zu Exspirationszeit (DGAI 2017). Es werden zwischen Oxygenierungsparameter und Ventilationsparameter unterschieden (Oczenski et al. 2012). Die Oxygenierungsparameter inspiratorische Sauerstofffraktion $\left(\mathrm{FIO}_{2}\right)$, PEEP und das I:E-Verhältnis werden zur Steuerung des arteriellen Sauerstoffpartialdrucks $\left(\mathrm{PaO}_{2}\right)$ herangezogen. Dahingegen nutzt man 
Tidalvolumen, Inspirationsdruck $\left(P_{\text {insp }}\right)$ und die Beatmungsfrequenz als Ventilationsparameter zur Steuerung des arteriellen Kohlenstoffdioxidpartialdrucks $\mathrm{PaCO}_{2}$. Indikationen für eine maschinelle Beatmung sind daher eine Ventilationsstörung, welche sich vornehmlich durch einen Anstieg des $\mathrm{PaCO}_{2}$ kennzeichnet und eine Oxygenierungsstörung, welche durch ein Abfallen des $\mathrm{PaO}_{2}$ auffällt (DGAI 2017).

Die Beatmungsform (Atemhilfe) beschreibt den Anteil der Atemarbeit, die der Patient leisten muss bzw. den Anteil, welcher vom Respirator übernommen wird (kontrolliert oder augmentiert), also die Interaktion zwischen Patienten und Respirator. Dabei stellt der Freiheitsgrad denjenigen Parameter dar, welcher nicht dem Respirator vorgegeben wird und sich folglich bei eingestellten Beatmungsparametern als Konsequenz aus den atemmechanischen Eigenschaften des Patienten ergibt (Oczenski et al. 2012). Je nach Anteil der Atemarbeit, die der Patient leisten muss, unterscheidet man zwischen kontrollierter oder mandatorischer Beatmung, bei der die gesamte Arbeit vom Respirator übernommen wird und assistierter oder augmentierter Beatmung, wenn nur ein Anteil vom Beatmungsgerät übernommen wird (DGAI 2017). Diese beiden Beatmungsformen stehen in Abgrenzung zur Spontanatmung, bei welcher der Patient die gesamte Atemarbeit übernimmt. Bei einer kontrollierten Beatmung $(\mathrm{CMV}=$ controlled mechanical ventilation $)$ erfolgt die Inspirationsarbeit unabhängig von eventuell vorhandenen Atembemühungen des Patienten allein durch den Respirator, weshalb auch keine Synchronisation von Patienten und Respirator angestrebt wird (Lumb und Nunn 2010).

Hierbei werden mehrere Formen der kontrollierten Beatmung unterschieden:

Bei der volumenkontrollierten Beatmung $(\mathrm{VCV}=$ volume controlled ventilation $)$ wird ein bestimmtes, meist nach einem Idealgewicht des Patienten gewähltes, Tidalvolumen mit einem konstantem Inspirationsfluss (daher auch flusskontrollierte Beatmung genannt) appliziert, wodurch der Inspirationsdruck den Freiheitsgrad dieser Beatmungsform darstellt (Oczenski et al. 2012). Im Druck-Zeit-Diagramm einer volumenkontrollierten Beatmung wird der Atemwegsdruck, welcher näherungsweise vor dem Tubus gemessen wird, gegen die Zeit aufgetragen (s. Abb. 2) (Oczenski et al. 2012). 


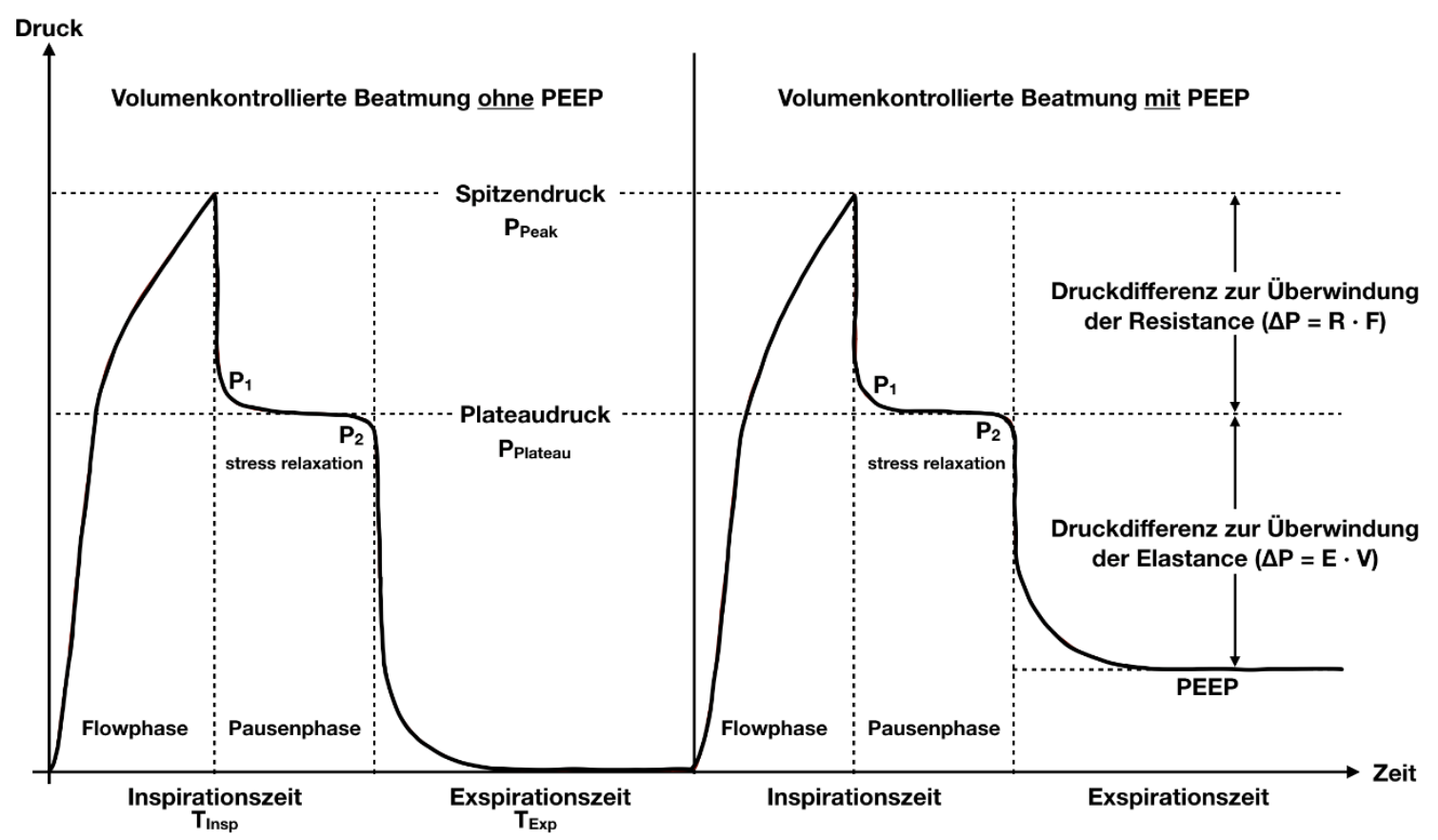

Abbildung 2: Phasen des Beatmungszyklus bei volumenkontrollierter Beatmung ohne PEEP (links) und mit PEEP (rechts)

Auf der Zeit-Achse werden jeweils nacheinander Inspirationszeit ( $\left.\mathrm{T}_{\mathrm{Insp}}\right)$ und Expirationszeit $\left(\mathrm{T}_{\text {Exp }}\right)$ durchlaufen und dabei applizierte Druckwerte (Druck-Achse) erreicht. Am Ende der Flussphase, in der ein zunehmender Druck einen Volumenstrom in die Lunge verursacht, wird der Spitzendruck $\left(\mathrm{P}_{\text {Peak }}\right)$ erreicht (Lumb und Nunn 2010). In der Pausen- oder Plateauphase fällt der Druck auf den Plateaudruck $\left(\mathrm{P}_{\text {Plateau }}\right)$ ab. Innerhalb dieser Phase erschlafft das Lungengewebe, sodass der gemessene vom anfänglichen Wert $\left(\mathrm{P}_{1}\right)$ auf den Druck-Wert kurz vor vor der Ausatmung $\left(\mathrm{P}_{2}\right)$ abfällt. Diese Druckdifferenz wird als stress relaxation bezeichnet (Cressoni et al. 2016). Wird ein PEEP am Beatmungsgerät eingestellt, fällt der Druck während der Exspirationsphase auf das eingestellte Niveau und nicht auf den atmosphärischen Druck ab. Die Druckdifferenz zur Überwindung der Elastance entspricht der treibenden Druckdifferenz (driving pressure) (Lumb und Nunn 2010).

Die Inspirationszeit wird in eine Flussphase und eine sich anschließende Pausen- oder Plateauphase eingeteilt. Während der Inspirationszeit steigt das Drucklevel ausgehend von einem eventuellen PEEP-Level kontinuierlich bis zu einem Spitzendruck $\left(P_{\max }, P_{P e a k}\right)$ an, welcher den maximalen Druck in den oberen Atemwegen darstellt (Larsen und Ziegenfuß 2013). Dieser Spitzendruck ist vom Inspirationsfluss, vom Tidalvolumen und den atemmechanischen Eigenschaften der Lunge abhängig. In der anschließenden Plateauphase wird der Plateaudruck erreicht $\left(P_{\text {Plateau }}\right)$. Die notwendige Druckdifferenz zur Überwindung der Elastance der Lunge wird treibende Druckdifferenz (driving pressure) genannt (Tonetti et al. 2017). Der Inspirationsfluss spiegelt dabei die Geschwindigkeit, mit der das Atemgas 
verabreicht wird, wider und wird über die endinspiratorische Pausenzeit eingestellt. Je niedriger der Inspirationsfluss, desto flacher der Druckanstieg. Wenn bei einem hohen Fluss das Volumen vor Ablauf der Inspirationszeit bereits appliziert wurde, entsteht eine anschlieBende no-flow-Phase, ehe die passive Exspirationsphase beginnt (Oczenski et al. 2012).

Bei der druckkontrollierten Beatmung (PCV = pressure controlled ventilation) erzeugt der Respirator einen konstanten Druck während der gesamten Inspirationszeit (Larsen und Ziegenfuß 2013). Dieser am Respirator eingestellte Inspirationsdruck $\left(P_{\text {insp }}\right)$ wird, ausgehend vom PEEP-Niveau je nach Druckanstiegsgeschwindigkeit, unterschiedlich schnell erreicht und dann für die jeweils eingestellte Inspirationszeit $\left(T_{\text {insp }}\right)$ gehalten. Das applizierte Tidalvolumen, welches hierbei den Freiheitsgrad darstellt, ergibt sich dabei aus der treibenden Druckdifferenz, der Inspirationszeit und den atemmechanischen Eigenschaften der Lunge. Daher nimmt das Tidalvolumen ab, wenn die Compliance abnimmt oder die Resistance zunimmt (Lumb und Nunn 2010).

\subsection{Positiver endexspiratorischer Druck (PEEP)}

\subsubsection{Entstehung eines PEEP}

Der positive endexspiratorische Druck (PEEP) wird je nach seiner Entstehung in einen extrinsischen und intrinsischen PEEP unterteilt (Lumb und Nunn 2010). Um einen extrinsischen PEEP zu generieren, wird mithilfe eines PEEP-Ventils am Beatmungsgerät während der gesamten Exspiration ein positiver Druck im Bezug zum atmosphärischen Druck aufrechterhalten. Dieser so erzeugte Druck wirkt als statische Druckgröße, die gleichmäßig über die gesamte Lunge wirkt. Werden allerdings unphysiologischerweise längere Inspirationszeiten als Exspirationszeiten am Beatmungsgerät gewählt (inverse ratio ventilation) und somit die Exspiration beendet, ehe das Lungenvolumen zur FRC zurückgekehrt ist, verbleibt ein endexspiratorischer Druck in den Alveolen, welcher als intrinsischer PEEP, dynamische Hyperinflation oder Auto-PEEP bezeichnet wird (Lumb und Nunn 2010).

\subsubsection{Auswirkungen des PEEP}

Bei Patienten, die intubiert und beatmet werden, wirkt ein PEEP einer Abnahme der funktionellen Residualkapazität entgegen, welche durch die Intubation (fehlender Glottisschluss), Analgosedierung und der Flachlagerung resultiert (Froese and Bryan 1974; DGAI 2017). Die Wirkungen eines PEEP werden oft mit alveolärer Rekrutierung in Verbindung gebracht, 
wobei ein ausreichend hoher Inspirationsdruck zuvor kollabierte (rekrutierbare) Lungenareale öffnet und ein entsprechend hoher PEEP-Wert diese offen hält (Oczenski et al. 2012). Eine kollabierte Alveole wird dabei als pulmonale Einheit definiert, welche kaum oder gar nicht mit Gas befüllt ist. Es werden dabei zwei Arten von Atelektasen unterschieden (Gattinoni et al. 2017a). Werden hauptsächlich auf Grund des Lungengewichtes kleine Atemwege zusammengedrückt (airway closure) und die nachgeschalteten Alveolen so durch Unterschreitung eines Alveolar-Verschlussdrucks nicht belüftet, spricht man von losen Atelektasen, welche zum Teil noch einen sehr geringen Gasinhalt aufweisen (Pelosi et al. 1999). Diese häufigere Form orientiert sich räumlich auf Grund unterschiedlicher Gravitationskräfte von nichtabhängigen (oben liegenden) zu abhängigen (unten liegenden) Lungenregionen (Crotti et al. 2001). Wird sämtliches Gas in den Alveolen bei mangelhafter Luftzufuhr mit der Zeit über die Blut-Luft-Schranke resorbiert, nennt man diese verklebte Atelektasen (Pelosi et al. 1999). Dies tritt sowohl in den abhängigen Lungenarealen, in denen der Inspirationsdruck nicht groß genug ist, die gravitationsbedingt zusammengefallenen Alveolen zu öffnen, und dort auf, wo Obstruktionen den Atemweg verschließen (Gattinoni et al. 2017a). Die Anzahl kollabierter Alveolen hängt in erster Linie von auf die Alveolen wirkenden Gravitationskräften ab (Pelosi et al. 1994; Gattinoni 1993; Cressoni et al. 2014a). Außerdem könnte auch die Interaktion angrenzender Alveolen eine Rolle spielen (Gattinoni et al. 2017a). Wenn also der Alveolar-Verschlussdruck unterschritten wird, muss bei der darauffolgenden Inspiration der Alveolar-Öffnungsdruck aufgebracht werden, um diese kollabierten Alveolarkompartimente erneut zu öffnen (Oczenski et al. 2012), indem mindestens folgende vier Kräfte überwunden werden (Gattinoni et al. 2017a): erstens die auftretende Oberflächenspannung (Ghadiali and Gaver 2008); zweitens der gravitationsbedingte Druck auf eine Alveole (Gattinoni 1993; Pelosi et al. 1994); drittens höchstwahrscheinlich ein durch Interaktion benachbarter Alveolen in einer Ebene gleicher Gravitationskraft (isogravimetrische Ebene) auftretender Druck (Cressoni et al. 2017) sowie viertens der Druck, welcher nötig ist, die Brustwand um dasselbe Volumen wie die Alveole zu heben (Cressoni et al. 2014a).

Nachdem die Lungeneinheiten während der Inspiration geöffnet wurden, müssen diese während der Exspiration mit einem PEEP offengehalten werden. Für das Offenhalten ist dabei eine kontinuierliche positive transpulmonale Druckdifferenz notwendig (Lumb und Nunn 2010). Nach dem Laplace-Gesetz ergibt sich, dass der Druck, welcher benötigt wird, um eine Lungeneinheit durch kontinuierliche Überwindung des Verschlussdrucks offenzuhalten, deutlich geringer ist, als der Öffnungsdruck. Ursächlich dafür ist, dass die inspiratorisch überwundene Oberflächenspannung nach der Öffnung dem offenhaltenden Druck nicht mehr entgegenwirkt (Gattinoni et al. 2017a). Wann immer ein end-exspiratorischer Druck 
appliziert wird, wird mittels des entsprechenden PEEP-Volumens ein gewisser Anteil der zuvor rekrutierten Alveolen offengehalten, aber auch die zuvor bereits geöffneten Alveolen zusätzlich mit Luft gefüllt (Gattinoni et al. 2017a). Dabei ist der Anteil des verabreichten PEEP-Volumens, welcher in die bereits geöffneten Alveolen strömt, sogar höher als der Teil, welcher in zuvor rekrutierte Alveolen strömt, bedingt durch starke Unterschiede in der Oberflächenspannung (Chiumello et al. 2016). Diese vermehrte Belüftung der geöffneten Alveolen sorgt so für eine (Über-)Dehnung (Gattinoni et al. 2017a).

Ein dabei insgesamt auf die Lunge wirkender globaler strain besteht aus einer dynamischen und einer statischen Komponente (Protti et al. 2013). Ein PEEP hält die Alveolen tonisch offen und bedingt durch das PEEP-Volumen wird so ein zusätzlich zum dynamischen strain, welcher durch das zyklische Applizieren des Tidalvolumens entsteht, ein statischer strain hervorgerufen. Dadurch vergrößert sich letztlich das in den Lungen verbleibende Volumen, da die funktionelle Residualkapazität (FRC) um das PEEP-Volumen erweitert wird, wodurch sich die Oberfläche zum Gasaustausch vergrößert (Protti et al. 2013).

Entsprechend dieser Wirkungen kann applizierter PEEP sowohl zu niedrig als auch zu hoch ausfallen. Ein zu niedriger PEEP ist ein PEEP unterhalb des kritischen Alveolarverschlussdrucks. Dadurch kommt es zum vermehrten atemzyklischen endexpspiratorischen Kollabieren und inspiratorischen Öffnen von Alveolarkompartimenten (Gattinoni et al. 2017a). Je nach Beatmungsform und atemmechanischen Eigenschaften des Patienten ergeben sich unterschiedliche Auswirkungen eines zu hohen PEEP-Levels auf das Lungengewebe (Oczenski et al. 2012). Bei volumenkontrollierter Beatmung resultiert eine Erhöhung des PEEP-Wertes in einer Erhöhung des endinspiratorischen Spitzen- und Plateaudrucks. Wird dadurch der endinspiratorische Plateaudruck über den obereren Inflektionspunkt verlagert, beginnt eine Überdehnung der Lunge, wobei Lungenschäden auftreten können (Oczenski et al. 2012). Bei einer druckkontrollierten Beatmung folgt aus einer isolierten Erhöhung des PEEP-Niveaus nicht zwangsläufig eine Überdehnung der Alveolen, da der insgesamt wirkende Inspirationsdruck konstant gehalten wird. Jedoch wird dadurch die treibende Druckdifferenz $(\Delta p=$ $\left.P_{\text {insp }}-P E E P\right)$ und folglich das applizierte Tidalvolumen gesenkt (Oczenski et al. 2012). 
Durch einen applizierten PEEP wird der intrathorakale Druck erhöht, wodurch auch der zentralvenöse Druck (ZVD) ansteigt (Larsen und Ziegenfuß 2013). Dadurch sinkt der venöse Rückstrom zum Herzen, welches eine Vorlastsenkung und dadurch einen Abfall des Herzzeitvolumens (HZV) und eine Minderdurchblutung der Organe bedeutet (DGAI 2017). Da der venöse Abfluss abgeschwächt wird, steigt durch die Rückstauung der Druck in den vorgeschalteten Organen an (zum Beispiel der intrakranielle Druck). Das Ausmaß dieser Nebenwirkungen hängt dabei folglich vom Volumenstatus und der Compliance des respiratorischen Systems des Patienten ab (Gattinoni et al. 2017b).

Ein PEEP verbessert letztlich die Oxygenierung durch zwei mögliche Mechanismen: Erstens führen offengehaltene Alveolen, welche zuvor kollabiert aber durchblutet wurden, zu einer Abnahme des intrapulmonalen Rechts-Links-Shunts (R-L-shunt). Zweitens reduziert ein PEEP durch den erhöhten intrathorakalen Druck das Herz-Zeit-Volumen und somit auch den R-L-shunt. Insgesamt wird das Ventilations-/Perfusionsverhältis und damit die Oxygenierung verbessert (Gattinoni et al. 2017a). Eine Erhöhung des PEEP führt somit zu einem Anstieg des $\mathrm{PaO}_{2}$, wobei dies mit den Risiken einer Lungenüberblähung und hämodynamischer Instabilität (Füllungsbehinderung primär des rechten Ventrikels) einhergehen kann (Gattinoni et al. 2017a; Dorinsky and Whitcomb 1983). Daher sollte neben dem $\mathrm{PaO}_{2}$ die Gewebeoxygnierung als eigentliche Zielgröße bedacht werden, da diese auf der einen Seite zwar durch den erhöhten $\mathrm{PaO}_{2}$ verbessert, auf der anderen Seite aber durch die verschlechterten hämodynamischen Parameter reduziert wird (Gattinoni et al. 2017a). Daher empfiehlt es sich, den PEEP möglichst optimal auf den jeweiligen Patienten einzustellen, indem eine individuelle Abschätzung erfolgt (DGAI 2017). Dies kann zum einen direkt durch bildgebende Verfahren, wie die besonders für kritisch kranke Patienten aufwändige Computertomografie (Gattinoni et al. 2006) oder die elektrische Impedanz-Tomographie (Frerichs et al. 2002), welche auch am Patientenbett möglich ist, durchgeführt werden. Zum anderen sind auch die nachfolgenden beispielsweise angeführten indirekten Verfahren möglich:

Die Optimierung der Oxygenierung kann durch definierte PEEP/ $/ \mathrm{FiO}_{2}$-Tabellen gemäß ARDS-Network (Brower et al. 2004) oder dem lung open ventilation strategy-Arm der LOVStudie erfolgen (Meade et al. 2008). Zwar ist diese Empfehlung, den PEEP nach der ARDSNetwork-Tabelle einzustellen, leicht umzusetzen, birgt allerdings den Nachteil, dass die atemmechanischen Eigenschaften des Patienten unberücksichtigt bleiben, wodurch diese Vorgehensweise lediglich zur Orientierung dienen sollte (DGAI 2017). 
Möglich ist auch die Optimierung der Compliance mittels Einstellung des PEEP ca. 2 $\mathrm{CmH}_{2} \mathrm{O}$ über dem unteren Infliktionspunkt der statischen/quasi statischen inspiratorischen Lungendehnungskurve (incremental PEEP trial) (Gattinoni 1993).

Eine weitere Optimierungsmöglichkeit der Compliance durch den PEEP kann sich auch nach dem oberen Infektionspunkt der statischen/quasi statischen inspiratorischen Lungendehnungskurve, bzw. nach der maximalen Änderung (point of maximum curvature, PMC) in der statischen/quasi statischen exspiratorischen Lungendehnungskurve (decremental PEEP trial) richten (Maltais et al. 1994).

Die Wahl des PEEP kann getroffen werden, wenn die Compliance bei minimal beeinträchtigter Sauerstofftransportkapazität (Herz-Zeit-Volumen multipliziert mit dem arteriellen Sauerstoffgehalt) des Kreislaufs maximiert wird (Suter et al. 1975).

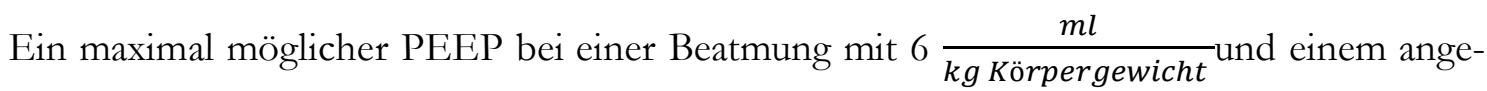
strebten inspiratorischem Atemwegsdruck von 28-30 $\mathrm{cm} \mathrm{H}_{2} 0$ kann gemäß der increased recruitment strategy der EXPRESS-Studie ausgewählt werden (Mercat et al. 2008). Außerdem kann dieser maximal mögliche PEEP auch erfasst werden, wenn anhand des stress-Indexes nach Ranieri die Rekrutierung atelektatischer Lungenareale in eine Überdehnung belüfteter Lungenareale übergeht (Grasso et al. 2004).

Die Einstellung des PEEP gleich (oder höher (maximal $15 \mathrm{~cm} \mathrm{H}_{2} 0$ )) dem absoluten endexspiratorischen Ösophagusdruck zur Vermeidung von negativen transpulmonalen Drücken ist eine weitere Möglichkeit einen adäquaten PEEP zu wählen (Talmor et al. 2008).

\subsection{Nebenwirkungen der maschinellen Beatmung}

Die Beatmung als Therapieform hat neben der beabsichtigten Wirkung, den Gasaustausch aufrechtzuhalten und die Atemarbeit zu reduzieren, mögliche hämodynamische und pulmonale Nebenwirkungen als Konsequenzen (Beitler et al. 2016). Bereits vor über 250 Jahren hat John Fothergill erkannt, dass die mechanische Beatmung (damals mit einem Blasebalg) Gefahren im Vergleich zur Beatmung mittels Mund-zu-Mund-Beatmung birgt (Fothergill 1744).

Ursächlich für einen dabei auftretenden Schaden ist die Interaktion zwischen Beatmungsgerät und Lunge. Demnach wird das Schadensausmaß sowohl von den Einstellungen des Beatmungsgerätes als auch von den Eigenschaften einer möglicherweise bereits 
vorgeschädigten Lunge abhängig sein (Gattinoni et al. 2017b). Eine mögliche Vorschädigung stellt das akute Lungenversagen dar (Suttorp et al. 2016). Das akute Lungenversagen (acute respiratory distress syndrome (ARDS), auch Schocklunge genannt) ist durch akut einsetzende, schwere Luftnot, eine Hypoxämie und bilaterale diffuse pulmonale Infiltrate charakterisiert, wodurch es zum respiratorischen Versagen kommt (Ranieri et al. 2012). Das ARDS wirkt sich dadurch auf die funktionelle Lungengröße, die intrinsischen Lungenelastitzitätseigenschaften und die Lungenhomogenität aus (Tonetti et al. 2017). Ursächlich für das ARDS können direkte (pulmonale) Schädigungsmechanismen wie beispielsweise eine bakterielle Pneumonie oder indirekte (extrapulmonale) Auslöser wie eine Sepsis sein (Suttorp et al. 2016). Eine als schädlich geltende maschinelle Beatmung kann in bereits vorgeschädigten und damit empfindlicheren Lungen einen bestehenden Schaden verstärken und womöglich leichter einen zusätzlichen Schaden verursachen (Suttorp et al. 2016).

Letztlich entstehen diese Nebenwirkungen durch zwei Hauptursachen: Eine unphysiologische Erhöhung des transpulmonalen Drucks und eine unphysiologische Erhöhung/Verminderung des intrapleuralen Drucks (Gattinoni et al. 2017b).

\subsubsection{Nebenwirkungen auf Grund des veränderten Pleuradrucks}

Ein erhöhter positiver Pleuradruck erhöht den zentralvenösen Druck und behindert so den venösen Rückstrom, wodurch das zirkulierende Blutvolumen reduziert wird (Gattinoni et al. 2017b). Die damit verbundenen Probleme treten besonders häufig auf, wenn der Pleuradruck nicht nur intermittierend positive Werte erreicht, sondern während des gesamten Atemzyklus positiv bleibt wie bei der Verwendung eines PEEP (Annat et al. 1983). Durch diese Venenstauung und Minderperfusion könnten vorgeschaltete Organe wie Niere, Leber und Darm geschädigt werden (Gattinoni et al. 2017b).

Ein verstärkt negativer Pleuradruck tritt beispielsweise bei einer Unterdruck-Beatmung auf. Der dabei verstärkte venöse Rückstrom führt über ein damit verbundenes erhöhtes Schlagvolumen zu einer erhöhten Wahrscheinlichkeit einer Lungenödembildung. Folglich sind die Veränderungen des intrapleuralen Drucks eher verantwortlich für die hämodynamischen Nebenwirkungen der maschinellen Beatmung (Gattinoni et al. 2017b). 


\subsubsection{Nebenwirkungen auf Grund des veränderten transpulmonalen Drucks}

Demgegenüber stehen die Veränderungen des transpulmonalen Drucks. Dieser als stress beschriebene Druck entspricht der mechanischen Spannung, welche auf das Alveolargewebe wirkt und folglich unmittelbar zu dessen Zerstörung beitragen kann. Dementsprechend steht der transpulmonale Druck eher in Beziehung zur einer sich ausbildenden beatmungsgerätassoziierten Lungenschädigung. Dabei ergeben sich die transpulmonalen Druckveränderungen $\left(\Delta P_{t p}\right)$ bei bestimmten Atemwegs-Druckdifferenzen $\left(\Delta P_{a w}\right)$ durch Multiplikation mit dem Verhältnis der Elastanzen der Lunge $\left(E_{w}\right)$ und des gesamten Atemapparates $\left(E_{t o t}\right)(G a t-$ tinoni et al. 2017b):

$$
\Delta P_{t p}=\Delta P_{a w} \cdot \frac{E_{w}}{E_{t o t}}
$$

\subsection{Beatmungsgerätassoziierte Lungenschädigung}

Der maschinellen Beatmung wurde seit ihrer Verwendung eine lungenschädliche Wirkung zugeschrieben, obwohl der heutige Begriff der beatmungsgerät-assoziierten Lungenschädigung (ventilator induced-lung injury (VILI) erst 1993 eingeführt wurde (Parker et al. 1993). Die Auswirkung einer solchen Schädigung reichen von Mikrofrakturen zu offenen Rupturen, von Zytokinproduktion zu Klustern von weißen Blutzellen und von einer veränderten Alveolarmembranpermeabiltität bis hin zu intra-alveoläreren Hämorrhagien (Tonetti et al. 2017). Der Begriff VILI fasst möglicherweise verschiedene pathophysiologische Mechanismen zusammen, welche zu diesen unterschiedlichen möglichen Manifestationen führen (Tonetti et al. 2017). Die dafür in Frage kommenden Auslöser werden im Folgenden betrachtet.

\subsubsection{Druck}

Dass exzessiver Druck zu makroskopischen Rupturen des Lungengewebes führt, zeigte sich besonders deutlich als ADRS-Patienten erstmals maschinell beatmet wurden (Kumar et al. 1973). Nachdem Tidalvolumina im Bereich von $15 \frac{\mathrm{ml}}{\mathrm{kg}}$ und Beatmungsfrequenzen zwischen 15-20 $\frac{1}{\min }$ gewählt wurden, traten nach kurzer Zeit (2-3 Tage) häufig Pneumothoraces und weitere klinisch wahrnehmbare Probleme der Luftentweichung (Pneumomediastinum, subkutanes Lungenemphysem, Gasembolien) auf (Gattinoni et al. 2017b). Diese klinischen Erscheinungen wurden als Barotrauma zusammengefasst (Kumar et al. 1973). Um das Auftreten eines solchen Barotraumas im Sinne einer lungenprotektiven Beatmung zu verringern, 
wird entsprechend der ARMA-Studie empfohlen, einen Atemwegsdruck von $30 \mathrm{~cm} \mathrm{H}_{2} \mathrm{O}$ als oberen Sicherheits-Grenzwert für eine maschinelle Beatmung nicht zu überschreiten (Tobin 2000). Bei einem normalen 70 kg Menschen während einer Anästhesie (FRC 2000 ml, Compliance $\left.80 \frac{\mathrm{ml}}{\mathrm{CmH}_{2} \mathrm{O}}\right)$ würde dieser Druck von etwa $30 \mathrm{~cm} \mathrm{H}_{2} \mathrm{O}$ mit einem Tidalvolumen von $2400 \mathrm{ml}$ einhergehen, wodurch sich ein strain von 1,2 entwickeln würde (Gattinoni et al. 2017b). Dieser strain bei einer Atemfrequenz von $15 \frac{1}{\min }$ zeigte über $54 \mathrm{~h}$ im Tierexperiment wenig schädliches Potenzial (Protti et al. 2011; Gattinoni et al. 2017b). Der aufgebrachte transpulmonale Druck wäre bei entsprechenden normalen Brustwand- und Lungenelastanzen $\left(\frac{E_{w}}{E_{t o t}} \approx 0,5\right)$ etwa $15 \mathrm{cmH}_{2} \mathrm{O}$. Allerdings würde ein solcher Druck-Grenzwert bei ARDS-Patieten mit einem variableren Verhältnis der Elastanzen $(0,2-0,8)$ nach der Formel

$$
\Delta P_{t r}=\Delta P_{a w} \cdot \frac{E_{w}}{E_{t o t}}
$$

zu einem transpulmonalen Druck zwischen 6-24 $\mathrm{cmH}_{2} \mathrm{O}$ führen (Gattinoni et al. 2017b). Ein transpulmonaler Druck von $24 \mathrm{~cm} \mathrm{H}_{2} \mathrm{O}$ entspricht näherungsweise dem Wert, der nötig ist, um die totale Lungenkapazität zu erreichen, welche im Tierexperiment zu 100\% tödlich endete (Protti et al. 2015). In entsprechenden Patientenkollektiven könnte ein gegebener Atemwegs-Druck von $30 \mathrm{~cm} \mathrm{H}_{2} \mathrm{O}$ also zu exzessiven strain oder zu einer Hypoventilation und damit einhergehender Hypoxämie führen (Gattinoni et al. 2017b). Folglich sollte bei der Betrachtung des Barotraumas besser der transpulmonale Druck (stress) statt des Atemwegsdruck herangeführt werden (Gattinoni et al. 2017b).

\subsubsection{Volumen}

Ein anderes Konzept rückte den Aspekt, dass die Lungenausdehnung (strain) einem applizierten Atemwegsdruck entgegenwirkt, in den Mittelpunkt. Hohe Atemwegsdrücke führten bei Beschränkung der Brustwandbeweglichkeit mittels Bandagierungen zu keinem exzessiven strain oder Lungenschäden. Dennoch wurden bei gleichen Atemwegsdrücken, während die Beweglichkeitseinschränkungen aufgehoben wurden, Lungenschäden beobachtet. Diese so als volumen-bedingt angenommenen Schäden wurden als Volutrauma bezeichnet (Dreyfuss et al. 1988). 
Um einem Volutrauma vorzubeugen, wird empfohlen das Tidalvolumen per idealem (vorhergesagtem) Körpergewicht (PBW) anzunehmen, um so das nötige Tidalvolumen einer erwarteten Lungengröße abzuschätzen (DGAI 2017). Allerdings gibt es eine enorme Varianz an ventilierbaren Lungenarealen beispielsweise in einer ARDS-Lunge (baby lung (Gattinoni and Pesenti 2005)), sodass eine solche Normalisierung als Surrogatparameter des Lungenstrain versagt. Um ein Volutrauma zu vermeiden, ist es folglich nicht sinnvoll lediglich das Tidalvolumen, sondern das Tidalvolumen in Bezug auf die FRC, also den strain zu betrachten. Bei der Vermeidung von zu hohem strain, sollte jedoch darauf geachtet werden, keine unerwünschten Nebenwirkungen wie beispielsweise Hypoxämie durch einen zu niedrigen strain zu induzieren (Gattinoni et al. 2017b).

Betrachtet man den transpulmonalen Druck (stress) statt des Atemwegsdrucks, welcher auf das Lungenskelett wirkt und den applizierten strain statt der Tidalvolumina, wird deren Zusammenhang durch folgende Gleichung ausgedrückt (Gattinoni et al. 2003; Gattinoni et al. 2017b):

$$
P_{t p}=E_{L_{s p e c}} \cdot \frac{\Delta V}{F R C}
$$

In der oben genannten Formel berechnet sich der transpulmonale Druck $\left(P_{t p}\right)$, indem die spezifische Lungenelastanz $\left(E_{L_{\text {spec }}}\right)$ mit dem Verhältnis von $\Delta V$ zu FRC multipliziert wird. $\Delta V$ stellt die Volumenänderung (durch das Tidalvolumen) in Bezug zum ungedehnten (ungestressten) Lungenvolumen während der Atemruhelage (FRC) dar (Gattinoni et al. 2017b):

$$
\text { stress }=E_{L_{\text {spec }}} \cdot \text { strain }
$$

Aus diesem Grund stehen stress und strain und damit auch ein Barotrauma und ein Volutrauma über die spezifische Lungenelastanz $\left(E_{L_{\text {spec }}}\right)$ als Proportionalitätsfaktor in Zusammenhang (Gattinoni et al. 2017b). Versuche deuten darauf hin, dass Barotraumata/Volutraumata dann auftreten, wenn zumindest Bereiche der Lunge die jeweilige totale Lungenkapazität (TLC) erreichen (Protti et al. 2016). Bei diesem Dehnungslevel ist das Kollagennetzwerk voll gedehnt und wirkt der weiteren Ausdehnung entgegen (Gattinoni et al. 2017b).

\subsubsection{Tidale Atelektasenbildung}

In einer ARDS-Lunge (baby lung) tragen eine Surfactant Dysfunktion und das Gewicht der ödematösen Lunge zur regionalen Atelektasenbildung bei (Pelosi et al. 1994; Taskar et al. 
1997). Um eine VILI in gesunden Tieren zu induzieren, sind Tidalvolumina von $30-40 \frac{\mathrm{ml}}{\mathrm{kg}}$ bei strain-Werten von 2-2,5 notwendig, wobei bei ARDS-geschädigten Lungen Tidalvolumina von $12 \frac{\mathrm{ml}}{\mathrm{kg}}$ aussreichend sind um eine VILI hervorzurufen (Gattinoni et al. 2017b). Die möglichen Veränderungen einer ARDS-Lunge (Surfactant-Mangel, Lungenödemformationen, Fibrose der extrazellulären Matrix) scheinen eher protektiv gegenüber strain zu sein, wodurch zusätzliche Faktoren wie die Inhomogenität einer Lunge möglicherweise als Ursache für die niedrigere Schadensschwelle in Frage kommen (Gattinoni et al. 2017b). Untersuchungen mittels Computer-Tomographien (CT) zeigen deutlich, dass vornehmlich die abhängigen Lungenpartien betroffen sind, bei gleichzeitiger relativer Aussparung der anderen Lungenareale (Suttorp et al. 2016). Dadurch entstehen starke regionale Unterschiede in den Dehnungs- und Strömungseigenschaften (Suttorp et al. 2016). Dadurch ergeben sich verschiedene Be- und Entlüftungszeiten der einzelnen Alveolarkompartimente (Otis et al. 1956). Man spricht von unterschiedlichen Zeitkonstanten $\tau(\tau=R \cdot C)$ und deshalb insgesamt von einer Multikompartment-Lunge (Otis et al. 1956). Auf Grund dieser unterschiedlichen Zeitkonstanten der Alveolen kommt es zu einer intrapulmonalen Gasumverteilung (= Pendelluft), da sich Luftströme von bereits geöffneten zu bislang noch geschlossenen Alveolen ausbilden. Dadurch führt Inhomogenität auf Grund unterschiedlicher Zeitkonstanten zu einer ungleichmäßigen Belüftung der Alveolen (Oczenski et al. 2012). An diesen Schnittstellen zwischen belüfteten und nicht-belüfteten Alveolen in einer inhomogenen Lunge treten Scherkräfte durch das zyklische Kollabieren und Wiedereröffnen von Alveolen auf (Mead et al. 1970). Diese Krafteinwirkung kann eine Lungenschädigung nach sich ziehen und zeigte sich experimentell durch einen deutlichen Anstieg inflammatorischer Zytokine, was insgesamt als Atelektrauma bezeichnet wird (Gattinoni et al. 2017b). Im theoretischen Modell führen diese Scherkräfte an den Schnittstellen zu einer Kerbwirkung (stress raiser), also einer lokalen Spannungsverstärkung (Mead et al. 1970; Otis et al. 1956). Diese immense Druckanstieg konnte im geringeren Ausmaß auch in CT-Analysen gezeigt werden (Cressoni et al. 2014b). Dadurch kommt es zu einer Vervielfachung des an der Kontaktstelle von geöffneten und kollabierten Alveolen wirkenden transpulmonalen Drucks, wodurch dieser lokal die entsprechenden Alveolarbereiche auf ihre Totalkapazität ausdehnt (Gattinoni et al. 2017b). Um das so ausgelöste Atelektrauma zu verhindern, gilt es folglich das Maß der Inhomogenität zu reduzieren, indem in rekrutierbaren Lungenarealen durch die Applikation eines ausreichenden PEEP die kollabierenden Alveolen während der Exspiration offengehalten werden (Gattinoni et al. 2017b). 


\subsubsection{Atemfrequenz}

Viele Studien beschränken sich bei der Entstehung einer VILI auf die statischen Komponenten eines Atemzuges (Tidalvolumen, Plateaudruck, PEEP). Allerdings sollte der dynamische Aspekt einer Beatmung ausgedrückt als Beatmungsfrequenz (Wie oft pro Minute wird eine potentielle Schädigung durch Druck oder Volumen appliziert?) und der inspiratorischen Flussrate (Wie schnell wird diese potentielle Schädigung appliziert?) nicht vernachlässigt werden (Gattinoni et al. 2017b). Allerdings spielt die Beatmungsfrequenz sicherlich auch eine Rolle bei der Entstehung einer VILI, zumal eine höhere Beatmungsfrequenz dafür sorgt, dass ein potentiell schädlicher Atemzug öfter pro Zeiteinheit verabreicht wird (Gattinoni et al. 2017b). Die Analogie zur Materialermüdung erscheint sinnvoll, da diese direkt von der Anzahl der stress-Zyklen abhängt, und zeigt die Funktion der Frequenz als Ko-Determinante einer VILI abzustecken (Gattinoni et al. 2017b; Protti et al. 2016). Außerdem zeigte sich in sowohl isolierten Lunge als auch in Versuchstieren, dass eine Frequenz-Reduktion mit einer reduzierten Auftretenswahrscheinlichkeit einer VILI korreliert (Hotchkiss, JR et al. 2000; Protti et al. 2016). Umgekehrt führt in erhöhten Druckbereichen eine Hoch-Frequenz-Ventilation mit niedrigen Tidalvolumina zu einem Schaden (Dreyfuss et al. 2015).

\subsubsection{Fluss}

Der Beitrag des inspiratorischen Flusses bei der Genese einer VILI liegt wahrscheinlich in der lokal intensivierten Konzentration von stress, welche durch die viskoelastischen Gewebeeigenschaften der Lunge begründet wird (Gattinoni et al. 2017b). Als vereinfachtes theoretisches Modell kann folgende Überlegungen angestellt werden: Man kann den inspiratorischen Fluss als Rate auffassen, in der ein bestimmter strain an der Lunge auftritt (Tonetti et al. 2017). Auf Grund der näherungsweise viskoelastischen Gewebeeigenschaften der Lunge führt eine höhere strain rate zu einer höheren sich entwickelnden Resistance in der extrazellulären Matrix (Tonetti et al. 2017). Dieser Prozess benötigt Energie, welche proportional zur strain rate zunimmt und innerhalb des Lungengewebes dissipiert (Tonetti et al. 2017). Wenn diese dissipierte Energie als Änderung des Drucks nach einer plötzlichen Unterbrechung des inspiratorischen Flusses (stress relaxation) aufgefasst wird (Milic-Emili 1989), zeigte sich, dass die Energie umso höher war, je höher die Atemfrequenz und die Lungenhomogenität war (Tonetti et al. 2017; Ganzert et al. 2009). Demnach hängt das Auftreten eines Lungenschadens nicht nur vom applizierten strain, sondern auch von dessen strain rate (strain pro Inspirationszeit) ab (Tonetti et al. 2017). Die strain rate ist also unabhängig vom applizierten 
strain ein Risikofaktor für die Entwicklung einer VILI (Protti et al. 2016). Wird die strain rate bei einem konstanten strain und einer gleichbleibenden Beatmungsfrequenz erhöht, zeigt sich ein deutlicher Anstieg der Rate an Lungenödemen und vorzeitigen Mortalität (Protti et al. 2016). Daher kann ein als unschädlich geltender strain-Wert bei einer niedrigen strain rate zu einem Lungenschaden führen, wenn die strain rate entsprechend erhöht wird (Protti et al. 2016). Experimentelle Versuche zeigen also, dass bei einem gegebenen Plateaudruck oder strain der inspiratorische Fluss definitiv eine Rolle bei VILI-Entstehung spielt (Gattinoni et al. 2017b; Protti et al. 2011; Protti et al. 2016; Rich et al. 2000; Garcia et al. 2008).

Das Lungen-Faser-Skelett besteht aus verzweigten und verknüpften („spaghettiartigen“) Elastin \& Kollagenfasern in einem hydratisierten Gel aus Proteoglykanen und anderen Proteine der extrazellulären Matrix (Toshima et al. 2004; Cavalcante et al. 2005). Die Applikation von strain führt zu einer stetigen Verschiebung dieser Bestandteile während der (Be-)Atmung, wodurch eine kontinuierliche atemzyklische Veränderung der physikalischen und chemischen Interaktionen in Form vom Aufbrechen und Neubilden dieser Bindungen zwischen diesen Komponenten hervorgerufen wird (Suki et al. 2011; Mijailovich et al. 1994; Yuan et al. 1997). Innere Reibungen (durch visköse Widerstände) führen zu einer Phasenverschiebung zwischen Belüftung und der daraus resultierenden Gewebedehnung, wodurch eine Dissipation eines Teiles der Energie über die Lunge entsteht (Hysteresis der Lunge) (Protti et al. 2016).

Durch eine Erhöhung der strain rate und die damit verbundene schnellere Geschwindigkeit der Lungenbelüftung kommt es zu einer ungleichmäßigeren Gewebedehnung und Gasverteilung sowie zu einer Zunahme der Scherkräfte parallel zur Oberfläche der Atemwege und Alveolen (Protti et al. 2016). Diese tangentiale Dehnung kann Entzündungsprozesse und somit langfristig zu Umbauprozessen in der extrazellulären Matrix der Alveolen führen (Garcia et al. 2008; Kotani et al. 2004; Pelosi und Rocco 2008). Die Alveolar-Einheiten der Lungen besitzen unterschiedliche mechanische Eigenschaften, besonders in Hinblick auf die Zeitkonstante $\tau$, sodass einige schnell-öffenende Einheiten einen großen Anteil des Tidalvolumens erhalten, während langsam-öffnende Einheiten einen geringeren Anteil erhalten (Otis et al. 1956). Diese Unterschiede werden besonders deutlich, wenn der Fluss ansteigt, was zu einer Überdehnung der schnell-öffnenden Alveolar-Einheiten führt (Colletti et al. 2011). Dadurch wird zum einen die Hysterese verstärkt und zum anderen führt dies zur anschließenden Umverteilung des Tidalvolumens in die langsam-öffnenden Lungenanteile, wodurch sich Pendelluft ergibt, welche wiederum Auswirkungen auf die stress relaxation hat 
(Protti et al. 2016). Durch diese inhomogene Ventilation entstehen analog zur inhomogenen Deformation lokale Stressverstärker (Protti et al. 2016). Beide Mechanismen, also die ungleichmäßigere Gewebedehnung und ungleichmäßigere Atemgasverteilung führen zu überdurchschnittlich hohen lokalen strain- und stress-Werten, welche das Risiko einer VILI deutlich erhöhen (Protti et al. 2016).

\subsubsection{Auswirkungen einer VILI}

All diese Faktoren stellen möglicherweise mechanische Auslöser für eine VILI dar (Tonetti et al. 2017). Während der Inspiration scheinen sich die Alveolen zu entfalten um elastische Dehnung und zellulären strain zu minimieren (Beitler et al. 2016). Wenn ein zellulärer strain auftritt, werden schnelle Lipidverschiebungen in die Plasmamembran induziert, um die Zelloberfläche zu erhöhen und somit Rupturen zu vermeiden und beschädigte Bereiche zu reparieren (Vlahakis et al. 2001; Vlahakis and Hubmayr 2005; Vlahakis et al. 2002). Werden die mechanischen Anforderungen an diese zytoprotektiven Mechanismen überschritten, führt eine weitere Inflation der Alveolen zu direktem Zell-strain, welche diese Zellen von der Basalmembran löst, epitheliale und endotheliale Zellverbindungen bricht und ein alveoläres und interstitielle Ödem bildet und somit histopathologisches Korrelat der VILI darstellt (Beitler et al. 2016).

Diese mechanische Schädigung löst sekundär eine lokale nicht-infektiöse Entzündungsreaktion in der Lunge (und gegebenenfalls eine systemische Entzündung) hervor und wird als Biotrauma bezeichnet (Beitler et al. 2016). Die Läsionen starten an den Schnittstellen von Strukturen unterschiedlicher Elastizität (stress raiser) (Cressoni et al. 2015). Je nach Ausmaß der applizierten mechanischen Leistung zeigt sich das Schadenspektrum von einer mechanischen Ruptur bis hin zu einer inflammatorsichen Reaktion, bedingt durch Aktivierung von Makrophagen (Frank et al. 2006), Neutrophilen (Belperio et al. 2002) und endotheliale und epitheliale Zellen (Vlahakis and Hubmayr 2003). Diese Veränderungen, die mit einem Integritätsverlust der extrazellulären Matrix einhergehen, können zum Beispiel über Toll-like-Rezeptoren 2 \& 4 eine Entzündungsreaktion hervorrufen (O'Neill 2005). In Kombination mit Mikro-Blutungen, welche durch Mikro-Frakturen in den Kapillaren entstehen (capillary leakage syndrome), ergibt sich das Ödem innerhalb der extrazellulären Matrix als Bestandteil der VILI (Protti et al. 2015). Die lokal freigesetzten Mediatoren können allerdings auch in den Systemkreislauf gelangen (spill over), wodurch systemische Entzündungsreaktionen 
(systemic inflammatory response snydrome (SIRS)) ausgelöst werden können (Ranieri et al. 2000; Beitler et al. 2016).

\subsection{Konzept der lungenprotektiven Beatmung}

Mit der Erforschung der auslösenden Faktoren einer VILI wurden auch Eindämmungsstrategien entwickelt, indem die Beatmungsparameter in entsprechenden empfohlenen Grenzen gehalten werden (Suttorp et al. 2016). Dies ist besonders entscheidend bei bereits vorgeschädigten Lungen wie der ARDS-Lunge (Suttorp et al. 2016). Hauptziel dabei ist die Auswahl niedriger Tidalvolumina und möglichst niedriger (Spitzen-)Drücke, um eine (zusätzliche) Lungenschädigung zu vermeiden, wobei ansteigende $\mathrm{PaCO}_{2}$-Werte toleriert werden (permissive Hyperkapnie) (Oczenski et al. 2012). Der Verwendung eines PEEP wird in diesem Zusammenhang seit den 1970er Jahren ein protektiver Effekt in Bezug auf die Entwicklung eines Lungenödems zugeschrieben (Webb and Tierney 1974). Als das Atelektrauma als eine der Hauptursachen für die VILI und ein angewendeter PEEP zur Verhinderung dieser Schädigung erkannt wurde, änderte sich das Ziel des PEEP von der bloßen Verbesserung der Oxygenierung zur Lungenprotektion (Gattinoni et al. 2017a). Trotz der Begrenzung der Beatmungsparameter muss beispielsweise bei ARDS-Patienten die Aufrechterhaltung eines suffizienten Atemgasaustauschs das oberste Ziel der Beatmung darstellen (Tobin 2000). Wenn also bei diesen Patienten das Tidalvolumen zur Verhinderung eines drohenden Volutraumas nicht weiter erhöht werden sollte, müssen andere Beatmungsparameter wie die Beatmungsfrequenz erhöht werden, damit ein ausreichender Gasaustausch stattfinden kann (Brower et al. 2000). In vielen Szenarien der Beatmungstherapie ist die alleinige Betrachtung eines einzelnen Beatmungsparameters nicht hinreichend, um das gesamte mögliche Schädigungspotenzial der maschinellen Beatmung zu erfassen, da zum Beispiel der angenommene potenziell schädliche Beitrag durch die Beatmungsfrequenz steigt (s. Kapitel 1.6.4). Dadurch stellt sich die Frage, welcher Beatmungsparameter den größten und entscheidenden Anteil zur Entstehung einer VILI beiträgt. Gibt es eine Priorisierung der Schädigungspotentiale einzelner Parameter oder sogar eine vereinheitlichende singuläre Größe?

Im Rahmen der Betrachtung der möglichen VILI-auslösenden Faktoren zeigten Tierexperimente, dass ein als tödlich geltender strain (größer als 2) nur dann tödlich verlief, wenn er mit

einer Atemfrequenz von 15 statt 3 oder $6 \frac{1}{\min }$ appliziert wurde (Guérin et al. 2016). 
Außerdem zeigte sich, dass dabei tidaler dynamischer strain eher einen Lungenschaden hervorruft als statischer PEEP-bedingter strain (Retamal et al. 2016). Zumal ein angenommener protektiver Effekt eines PEEP sich in erster Linie durch die meist begleitende Reduktion des Tidalvolumens bemerkbar macht (Tonetti et al. 2017). Des Weiteren spielt ebenfalls die strain rate (ausgedrückt als inspiratorischer Fluss) eine Rolle als mögliche Determinante der VILI (Marini 2016). Fasst man diese Erkenntnisse zusammen, liegt der Schluss nahe, dass die aufgeführten möglichen auslösenden Beatmungsparameter für eine VILI (Volumen, Druck, Frequenz, Fluss, PEEP) als Teile einer einzelnen physikalischen Größe mit unterschiedlich starkem Einfluss aufgefasst werden können (Gattinoni et al. 2017b; Cressoni et al. 2014b). Diese Größe könnte die durch das Beatmungsgerät applizierte mechanischen Leistung sein (Tonetti et al. 2017).

\subsection{Die mechanische Leistung der maschinellen Beatmung}

Die mechanische Leistung ist die pro Zeiteinheit veränderte Energiemenge. Bei der maschinellen Beatmung entspricht diese Energiemenge der verrichteten Druck-Volumen-Arbeit, also dem Produkt aus absolutem Druck und der dabei auftretenden Volumenänderungen (Protti et al. 2015). Der zu einem beliebigen Zeitpunkt auf das respiratorische System wirkende Druck besteht aus mehreren Druckkomponenten, welche notwendig sind, die bei der Beatmung auftretenden Widerstände zu überwinden (Gattinoni et al. 2016). Diese Widerstände ergeben sich zum einen durch die elastischen Rückstellkräfte, welche durch die Elastance ausgedrückt werden und den Strömungswiderstand also der Resistance der Lunge und zusätzlich durch einen PEEP-Wert (Gattinoni et al. 2016). Im Folgenden werden die sich daraus ergebenen drei Druckkomponenten besprochen (Gattinoni et al. 2016):

Erstens auf Grund der elastischen Rückstellkräfte ergibt sich aus der Formel für die Elastance des respiratorischen Systems $\left(E_{r s}\right)$ eine dynamische Druckkompenente $\left(\Delta P_{r s, d y n}\right)$ :

$$
E_{r s}=\frac{\Delta P_{r s, d y n}}{\Delta V}=\frac{P_{p l a t}-P E E P}{\Delta V} \Leftrightarrow \Delta P_{r s, d y n}=P_{\text {plat }}-P E E P=E_{r s} \cdot \Delta V
$$

Zweitens auf Grund des Strömungswiderstandes in den Atemwegen $\left(R_{a w}\right)$ ergibt sich daher als resistive Druckkomponente $\left(\Delta P_{r s, r e s}\right)$ : zur Überwindung der Reibungsarbeit:

$$
R_{a w}=\frac{\Delta P_{r s, r e s}}{F}=\frac{P_{\text {peak }}-P_{\text {plat }}}{F} \Leftrightarrow \Delta P_{r s, r e s}=P_{\text {peak }}-P_{\text {plat }}=R_{\text {aw }} \cdot F
$$


Drittens ergänzt ein eingestellter PEEP den herrschenden Druck um eine statische elastische Komponente $\left(\triangle P_{L, P E E P}\right)$. Das Hinzufügen des PEEP zur Formel der mechanischen Leistung erscheint sinnvoll, wenn man die physikalischen Besonderheiten eines elastischen Systems betrachtet. Gemäß des Hook'schen Gesetzes ist die Kraft $\left(F_{\text {Hook }}\right)$, um einen elastischen Körper um die Länge $x_{H o o k}$ auszulenken, direkt proportional einer Konstanten $k_{H o o k}$, welche die intrinsischen Elastizitätseigenschaften des Systems widerspiegelt (Tonetti et al. 2017):

$$
F_{\text {Hook }}=k_{\text {Hook }} \cdot x_{\text {Hook }}
$$

Daher ist die Kraft (und somit auch die Energie), um einen elastischen Körper wie die Lunge zu dehnen, strikt abhängig vom Grad der Auslenkung von der Ruheposition des Systems (FRC). Folglich repräsentiert das Produkt aus PEEP und appliziertem Tidalvolumen die Energiemenge, die überschritten werden muss, um jedes Tidalvolumen zu verabreichen. AuBerdem ist die Energie nicht das Produkt aus Druckänderungen und Volumenänderungen, sondern aus absoluten Drücken und Volumenänderungen, sodass der PEEP in der Leistungsgleichung berücksichtigt werden muss (Tonetti et al. 2017). Insgesamt ergibt sich daher für den auf das respiratorische System wirkenden Druck $\left(P_{r s}\right)$ :

$$
P_{r s}=\Delta P_{r s, d y n}+\Delta P_{r s, r e s}+\Delta P_{r s, P E E P}=E_{r s} \cdot \Delta V+R_{a w} \cdot F+P E E P
$$

In Bezug auf die Volumenänderung betrachtet man das jeweilige Tidalvolumen, sodass sich für die Energie pro Atemzug im respiratorischen System $\left(E_{r s, b r e a t h}\right)$ ergibt:

$$
E_{r s, \text { breath }}=\Delta V \cdot \Delta V \cdot E_{r s} \cdot \frac{1}{2}+\Delta V \cdot R_{a w} \cdot F+\Delta V \cdot P E E P
$$

Dabei beinhaltet diese Energiemenge mehrere Komponenten. Eine durch den PEEP und das entsprechende PEEP-Volumen hervorgerufene statische Komponente, welche eine potenzielle Energie darstellt. Zudem eine durch die treibende Druckdifferenz und das sich dadurch ergebende Tidalvolumen dynamische zyklische Komponente, welche eine kinetische Energie darstellt (Protti et al. 2015).

Der erste Teil der Gleichung muss dabei um den Faktor 0,5 angepasst werden, um so eine bessere Annäherung an das Flächenintegral unter der Druck-Volumen-Kurve zu erhalten (Gattinoni et al. 2016). Da der Inspirationsfluss dem Tidalvolumen pro Inspirationszeit entspricht, ergibt sich:

$$
E_{r s, \text { breath }}=\Delta V^{2} \cdot E_{r s} \cdot \frac{1}{2}+\Delta V \cdot\left(R_{a w} \cdot \frac{\Delta V}{T_{\text {insp }}}\right)+\Delta V \cdot P E E P
$$

Daher gilt weiterhin: 


$$
E_{r s, \text { breath }}=\Delta V^{2} \cdot\left(E_{r s} \cdot \frac{1}{2}+\frac{1}{T_{\text {insp }}} \cdot R_{a w}\right)+\Delta V \cdot P E E P
$$

Um die Inspirationszeit $\left(T_{\text {insp }}\right)$ mithilfe des I:E-Verhältnis und der Atemfrequenz (RR) auszudrücken, ergibt sich aus den folgenden Beziehungen $\frac{T_{\text {insp }}}{T_{\text {exp }}}=I: E$ sowie $T_{\text {insp }}+T_{\text {exp }}=$ $T_{\text {tot }}$ durch mathematische Umformungen zunächst $T_{\text {insp }}=T_{\text {tot }} \cdot\left(\frac{I: E}{(1+I: E)}\right)$. Weiterhin kann $T_{\text {tot }}$ als $T_{\text {tot }}=\frac{60}{R R}$ ausgedrückt werden (Gattinoni et al. 2016).

Insgesamt ergibt sich somit:

$$
E_{r s, \text { breath }}=\Delta V^{2} \cdot\left(E_{r s} \cdot \frac{1}{2}+R R \cdot \frac{(1+I: E)}{60 \cdot I: E} \cdot R_{a w}\right)+\Delta V \cdot P E E P
$$

Um die Volumina in $l$ und die Drücke in $\mathrm{CmH}_{2} \mathrm{O}$ anzugeben, wird dazu ein Anpassungsfaktor von 0,098 benötigt. Um anschließend die Energie pro Zeiteinheit, also die Leistung, welche auf das respiratorische System wirkt $\left(\right.$ Power $\left._{r s}\right)$, zu erhalten, muss man die Energie pro Atemzug mit der Atemfrequenz multiplizieren (Gattinoni et al. 2016):

$$
\text { Power }_{r s}=0,098 \cdot R R \cdot\left\{\Delta V^{2} \cdot\left[\frac{1}{2} \cdot E_{r s}+R R \cdot \frac{(1+I: E)}{60 \cdot I: E} \cdot R_{a w}\right]+\Delta V \cdot P E E P\right\}
$$

Vergleicht man den Einfluss der einzelnen Komponenten ist die mechanische Leistung proportional zum Quadrat des Tidalvolumens $\left(\Delta V^{2}\right)$ und des inspiratorischen Flusses $\left(\right.$ Flow $\left.^{2}\right)$ sowie zur der 1,4 Potenz der Atemfrequenz $\left(R R^{1,4}\right)$ und linear abhängig vom PEEP, der Elastance und Resistance. Dadurch könnte möglicherweise eine Prioritätsrangfolge erstellt werden, inwieweit man welchen Parameter zur Reduktion der schadensverursachenden mechanischen Leistung reduzieren könnte (Gattinoni et al. 2017b). 


\section{$2 \quad$ Material und Methoden}

\subsection{Ziele der Arbeit}

In dieser Dissertation wollte ich tierexperimentell untersuchen, ob der applizierte PEEP tatsächlich als Teil der verabreichten mechanischen Leistung zum beschriebenen Ergotrauma beiträgt, indem die Auswirkungen unterschiedlich hoher PEEP-Werte auf einen sich möglicherweise entwickelnden Lungenschaden analysiert wurden. So soll überprüft werden, ob ein applizierter PEEP mehr schädliches Potenzial aufweist, als dem bisher als protektiv geltendem PEEP zugeschrieben worden ist.

\subsection{Studiendesign}

Dazu wurden 40 weibliche Hausschweine über einen Zeitraum von 50 Stunden maschinell beatmet, indem mithilfe der zuvor ermittelten FRC das Tidalvolumen so gewählt wurde, dass sich für jedes Tier ein strain von 1,0 ergab (Tidalvolumen = FRC). Sämtliche anderen Beatmungsparameter wurden vergleichbar und konstant gehalten, sodass die sich lediglich im gewählten PEEP-Wert unterscheidenden Versuchstiere in sechs Gruppen mit PEEP-Werten $0,4,7,11,14$ und $18 \mathrm{~cm} \mathrm{H}_{2} 0$ eingeteilt wurden. Atemfrequenz und Tidalvolumen wurden letztlich so gewählt, dass sie entsprechend vorheriger Studien für sich genommen nicht tödlich sind, aber in Kombination mit einem entsprechenden PEEP-Wert eine mechanische Leistung ergaben, welche in einem Bereich lag, in dem der bisher angenommene kritische Schwellenwert zum Auftreten erheblicher Schäden liegt. Die Werte für Beatmungsparameter waren dabei weit oberhalb dessen, was als lungenprotektive Beatmung gilt, um in dem vergleichsweise kurzem Beatmungszeitraum in lungengesunden Versuchstieren einen sicher nachweisbaren Schaden zu erzeugen. Dabei wurden pathologische Untersuchungen und die Entwicklung von Flüssigkeitsansammlungen in der Lunge als Marker für eine aufgetretene Beschädigung der Lunge herangeführt, aber auch kritisch beleuchtet. Weiterhin wurden die atemmechanischen Parameter (wie die mechanische Leistung) analysiert, um einen möglichen Grenzwert der mechanischen Leistung für das Auftreten eines beatmungsgerätassoziierten Lungenschadens zu identifizieren, sodass bei der Patientenanwendung mithilfe des Konzepts der mechanischen Leistung und des Grenzwertes der Leistung ein sicheres Kriterium vorliegt, bis wann eine bestimmte Konstellation maschineller Beatmungsparameter eine Lungenprotektion gewährleisten kann. 


\subsection{Allgemeine Anästhesie}

\subsubsection{Prämedikation und Narkoseeinleitung}

Die Versuchstiere erhielten eine Prämedikation durch $4 \frac{m g}{k g \text { Körpergewicht }}$ Azaperon (Janssen, Neuss, Deutschland) und $20 \frac{\mathrm{mg}}{\mathrm{kg} \mathrm{Körpergewicht}}$ Ketamin (Inresa, Freiburg, Deutschland) sowie $5 \frac{\mathrm{mg}}{\mathrm{kg} \mathrm{Körpergewicht}}$ Midazolam (Ratiopharm, Ulm, Deutschland) mittels intramuskulärer Injektion in den Nacken auf beiden Seiten. Dabei wurde darauf geachtet, ein ruhiges und weitestgehend stressfreies Narkose-Umfeld sicherzustellen. Anschließend erfolgte die peripher-venöse Kanülierung (20-22 Gauge) am Ohr. Während des gesamten Experimentes wurde mithilfe von Wärmedecken Hyper- und Hypothermien vermieden. Es erfolgte eine kontinuierliche Überwachung der peripheren Sauerstoff-Sättigung $\left(\mathrm{SpO}_{2}\right)$ mittels Pulsoxymetrie am Schwanz des Versuchstieres. Außerdem wurde die Herztätigkeit mithilfe eines Elektrokardiogramms (EKG) überwacht. Des Weiteren wurden die Schweine zu Beginn und am Ende gewogen und die Länge sowie der Thoraxumfang der Tiere initial bestimmt.

Zur Präoxygenierung erhielten die Tiere mittels eines Nasenkonus 100\% Sauerstoff bei 15 $\frac{l \mathrm{O}_{2}}{\mathrm{~min}}$. Es folgte die intravenöse Gabe von Sufentanyl (Rotex medica, Trittau, Deutschland) zur Analgosedierung von etwa 1,0-1,2 $\frac{\mu g}{\mathrm{~kg} \text { Körpergewicht }}$. Dazu eine intravenöse Gabe von Propofol (Propofol 2\%) als Hypnotikum von 2-3 $\frac{\mathrm{mg}}{\mathrm{kg} \mathrm{Körpergewicht}}$ als langsamen Bolus bis zu einer adäquaten Narkose-Tiefe. Die Narkose-Tiefe wurde initial und mehrfach im Verlauf durch eine Reflextestung am Schweinefuß festgestellt.

\subsubsection{Intubation und Ventilation zu Beginn}

Die Versuchstiere wurden in Bauchlage spontanatmend endotracheal intubiert. Dabei wurde zur Reduktion der Atemwegswiderstände der jeweilig größtmögliche Durchmesser des Endotrachealtubus gewählt (Durchmesser für den Tubus lagen dabei zwischen 5,0-6,5 mm). Dabei wurde, nachdem das Laryngoskop vorgeschoben wurde, die Epiglottis auf die Spitze des Laryngoskopes (meist unter Zuhilfenahme eines Führungsstabes) gehoben, um so die Stimmbänder darzustellen und den Tubus sicher vorzuschieben. Die Messung von endtidalem Kohlenstoffdioxid diente dabei als sicheres Zeichen einer korrekten endotrachealen Intubation (Larsen 2018). Nach erfolgter Intubation wurden die Versuchstiere zunächst an das 
maschinelle Beatmungsgerät „AVEA (R16952) (Carefusion)“ angeschlossen und mit einer volumenkontrollierten Beatmung mit folgenden Parametern beatmet: $\mathrm{FiO}_{2} 40 \%$, Tidalvolumen von etwa $10 \frac{\mathrm{ml}}{\mathrm{kg} \mathrm{Körpergewicht}}$, Atemfrequenz zwischen 15-18 Atemzüge pro Minute um ein endtidales $\mathrm{CO}_{2}\left(e t \mathrm{CO}_{2}\right)$ zwischen $35-45 \mathrm{mmHg}$ und ein Inspirations- zu Exspirations-Verhältnis von 1:2 zu gewährleisten.

\subsubsection{Anästhesie-Verlauf}

Zur Aufrechterhaltung der Narkose wurde eine kontinuierliche intravenöse Infusion von Propofol $(2 \%) 6-9 \frac{\frac{m g}{\text { kg Körpergewicht }}}{h}$ in Kombination mit 1,2 - 1,5 $\frac{\frac{m g}{\mathrm{~kg} \mathrm{Körpergewicht}}}{h}$ Midazolam verabreicht. Dabei wurden keine Inhalationsnarkotika zur Aufrechterhaltung der Anästhesie verwendet. Zur weiteren Schmerzkontrolle erhielten die Versuchstiere eine Sufentanyl-Infusion von $2-3 \frac{\frac{\mu g}{\text { kg Körpergewicht }}}{h}$.

Um eine stabile Hämodynamik mit einem arteriellen Mitteldruck von 60-90 $\mathrm{mmHg}$ aufrechtzuhalten, erhielten die Versuchstiere neben den kontinuierlichen $\left(2-3 \frac{\frac{\mathrm{ml}}{\mathrm{kg} \text { Körpergewicht }}}{\mathrm{h}}\right)$ Voll-Elektrolyt-Lösungen (Sterofundin 1/1 (B. Braun Melsungen, Deutschland) mit Zusatz von $100 \mathrm{ml}$ Glucose 40 \% (G40 \%) und $100 \mathrm{mEq}$ Kalium sowie zwei Gramm Calcium. Bei Bedarf wurden $100 \mathrm{ml}$ Gelafundin-Infusionen (Gelafundin 4 \%, Braun, Deutschland) verabreicht. Darüber hinaus wurde gegebenenfalls eine zusätzliche Noradrenalin-Infusion hinzugezogen.

Zur Antikoagulation wurden anfangs 10.000 I.E. Heparin appliziert und eine zusätzliche Heparin-Infusion von etwa 400 I.E. pro Stunde. Zu Beginn des Experiments wurden die Versuchstiere mit Amoxicillin (15 $\frac{\frac{m g}{\mathrm{~kg} \mathrm{Körpergewicht}}}{d}$ als i.m. Injektion) als Antibiose prophylaktisch behandelt. 


\subsection{Katheterisierung}

Nach einer direkten Blasenkatheterisierung zur Drainage der Schweineblase wurde ein arterieller Zugang in der A. femoralis mithilfe sonographie-gestützter perkutaner Punktion in Rückenlage des Schweins eingebracht. Ein zentral-venöser Katheter sowie eine zentral-venöse Schleuse wurden transkutan über die $V$.jugularis externa in das Gefäßsystem platziert. Durch die Schleuse wurde ein Pulmonaliskatheter (Swan-Ganz-Katheters) eingesetzt, wodurch die Drücke in der Pulmonalarterie (PAP) direkt und die Füllungsdrücke des linken Herzens, der Lungenkapillaren-Verschlussdruckes (PCWP, Wedge-Druck) und das Herzzeitvolumens indirekt gemessen wurden. Dabei wurde das Schlagvolumen mithilfe der Thermodilutionsmethode bei Verwendung eines Termistor-Katheters berechnet und das Herzzeitvolumen durch Multiplikation mit der gemessenen Herzfrequenz bestimmt (Larsen 2018). Das Einsetzen und (wiederholte) Kalibrieren eines ösophagealen Katheters ermöglichte durch Abschätzung des intrapleuralen Drucks die Berechnung des transpulmonalen Druckes. Außerdem wurde zusätzlich eine HZV-Messung mittels Pulskonturanalyse durchgeführt. Dabei wurde das PiCCO-Gerät „PulsioFlex (Pulsion Medical System)“ durch wiederholte transpulmonale Thermodilutionsmessung kalibriert (Larsen 2018). Ein kalter Kochsalzbolus wurde dafür durch den zentralen Venenkatheter injiziert und die sich daraus ergebende Temperaturverlaufskurve im arteriellen System (A. femoralis) durch einen Thermodilutionskatheter im arteriellen Zugang registriert (Larsen 2018). Anschließend wurde mithilfe der Herzfrequenz, des Flächenintegrals unter dem systolischen Teil der arteriellen Druckkurve, der Compliance der Aorta und der Form der Blutdruckkurve kontinuierlich das HZV berechnet. Mithilfe dieser Messwerte berechnet das Messgerät außerdem die kardiale Vorlast, das intrathorakale Blutvolumen und das extravasale Lungenwasser (EVLW). Das Monitoring wurde unter Verwendung des Gerätes „Solar M8000 (GE Healthcare)“ durchgeführt.

Einen zeitlichen und räumlichen Ablauf (Versuchsaufbau) bietet Abbildung 3 . 

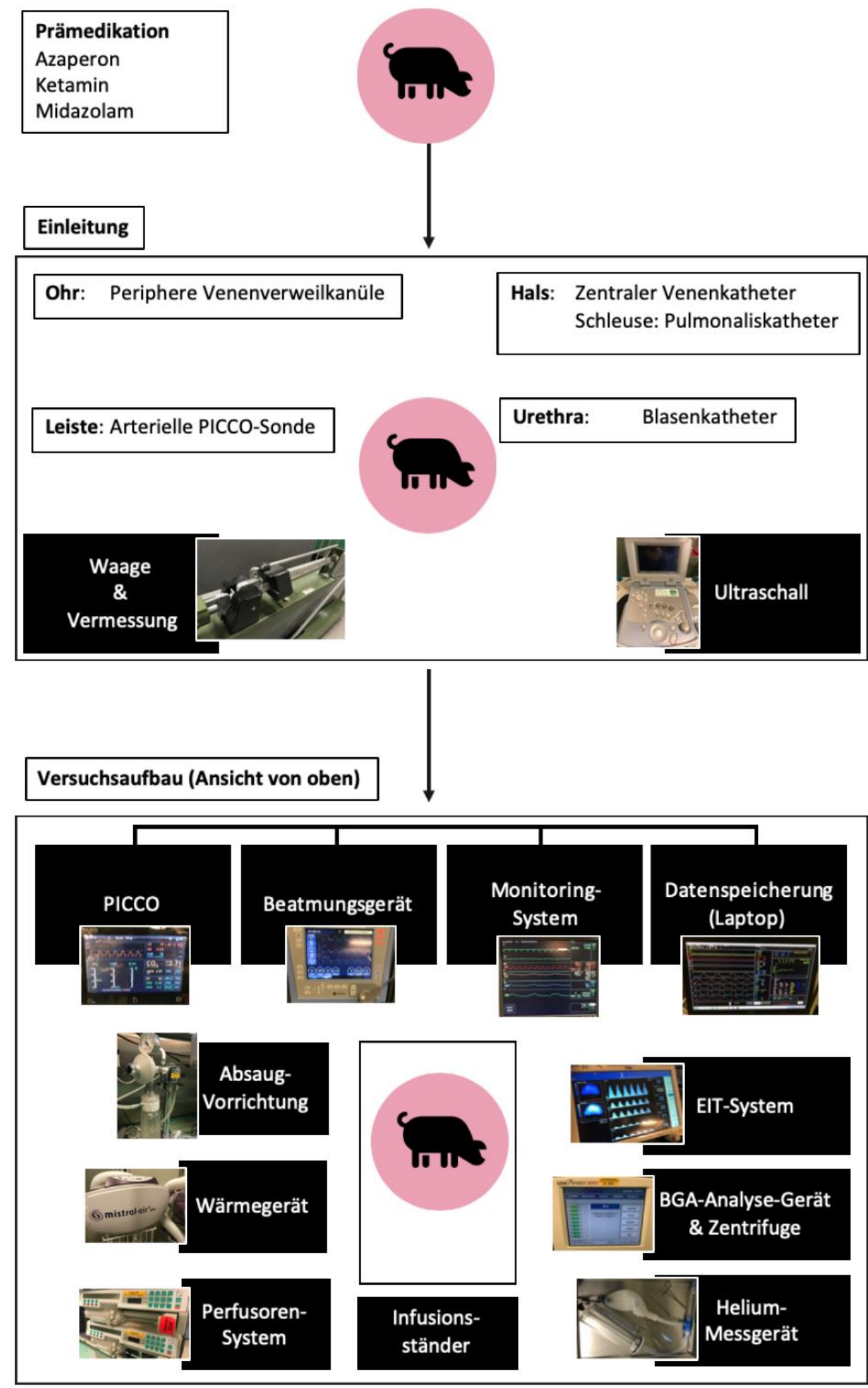

Abbildung 3: Ablauf der Katheterisierung und Versuchsaufbau

Die Abbildung zeigt eine schematische Darstellung des Ablaufes und des Versuchsaufbaus. Der gezeigte Ablauf umfasst die Prämedikation, die Einleitung und Katheterisierungen. Der Versuchsaufbau in der Aufsicht dient als Übersicht über die verwendeten Geräte zur Überwachung und für die Messungen. 


\subsection{Atemmechanische Messreihen und Auswertungen}

\subsubsection{FRC-Messung}

Nachdem die Versuchstiere erneut in Bauchlage gebracht wurden, erfolgte eine endotracheale Absaugung und ein zweiminütiges Rekrutierungs-Manöver in Form einer druckkontrollierten Ventilation mit einem Inspirationsdruck von $30 \mathrm{~cm} \mathrm{H}_{2} \mathrm{O}$ und einer Atemfre-

quenz von $10 \frac{1}{\min }$ bei sonst konstanten Parametern. Zunächst wurde die funktionelle Residualkapazität mittels Helium-Einwaschmethode bestimmt. Dabei wurde die Helium-Konzentration eines mit einem bestimmten Volumen gefüllten Beatmungsbeutels $(1100 \mathrm{ml})$ mithilfe des Gerätes Digital Gas Analyzer (DE-OX) gemessen. Nach 15 Atemzügen, welche durch den Beatmungsbeutel durchgeführt wurden, wurde die Helium-Konzentration innerhalb des Beatmungsbeutels erneut ermittelt und daraus die funktionelle Residualkapazität bestimmt (Chiumello et al. 2008b).

\subsubsection{Protektive Beatmung}

Anschließend wurde das Tidalvolumen im Beatmungsgerät entsprechend der ermittelten FRC aus der Helium-Einwaschmethode gesetzt, sodass bei Übereinstimmung von Tidalvolumen und FRC ein strain von 1,0 entstand. Die Beatmungsfrequenz wurde auf 20 Atemzüge pro Minute gestellt und ein als protektiv angenommener PEEP von $5 \mathrm{cmH}_{2} \mathrm{O}$ eingestellt. Daraufhin wurde nach einer trachealen Absaugung ein Rekrutierungs-Manöver mit einer druckkontrollierten Beatmung bei einem hohen Inspirationsdruck von $30 \mathrm{~cm} \mathrm{H}_{2} \mathrm{O}$ (einschließlich gewähltem PEEP) bei einer Atemfrequenz von 10 Atemzügen pro Minute durchgeführt. Anschließend folgten bei volumenkontrollierter Beatmung mit der FRC entsprechendem Tidalvolumen und gesetztem PEEP von $5 \mathrm{~cm} \mathrm{H}_{2} \mathrm{O}$ zwei inspiratorische und zwei exspiratorische Okklusionsmanöver, ehe der PEEP auf $0 \mathrm{~cm} \mathrm{H}_{2} \mathrm{O}$ gesetzt wurde und diese Okklusionsmanöver erneut durchgeführt wurden. Einem weiteren Rekrutierungs-Manöver schlossen sich zwei Bestimmungen der Ruhedehnungskurve im Bereich des gesetzten Tidalvolumens an. Anschließend wurden diese aufgezeichneten Messwerte mithilfe der Software Diadem 2015 von National Instruments ausgewertet. Dabei wurden für die mehrfach erhobenen Werte die arithmetischen Mittelwerte berechnet und für die Berechnungen weiterer atemmechanischer Größen verwendet. Nach Beendigung dieser Messung wurde um den rasierten Thorax der Elektrodengürtel für die elektrische Impedanztomografie (EIT) angelegt und das EIT-Gerät Infinity C500 (Dräger) kalibriert und eine kontinuierliche Messung bis 
zum Ende der Experimente durchgeführt. Die EIT ist ein nichtinvasives und ohne Strahlenbelastung auskommendes bildgebendes Untersuchungsverfahren, dessen Funktionsprinzip auf der Erfassung der bioelektrischen Eigenschaften des Lungengewebes beruht, wobei die Änderungen der regionalen elektrischen Widerstände (= Impedanzen) im Thoraxquerschnitt bestimmt werden (Hahn et al. 1998).

\subsubsection{Baseline-Messung und Messreihen}

Nach diesen Messungen wurde der PEEP des Versuchstieres auf den jeweiligen Wert entsprechend der zufällig zugewiesenen Gruppe eingestellt, sodass mit der Initialmessung begonnen werden konnte. Der Ablauf einer Messreihe umfasste zunächst die arterielle, venöse und gemischt-venöse Blutgasanalyse und kapnometrische Berechnungen, Sammlung von Plasma- und Urinproben, die Flüssigkeitsbilanzierung des Schweins, die Erfassung der hämodynamischen Parameter mit Thermodilutionsverfahren und die Ermittlung der respiratorischen Größen wie bei der protektiven Beatmung. In sechsstündigen Intervallen wurden diese Messungen wiederholt, bis der Endpunkt von $50 h$ und die damit verbundene Abschlussmessung erreicht wurde, ehe die Schweine mit Thiopental und einer Kalium-Injektion eingeschläfert wurden.

Eine Übersicht des zeitlichen Ablaufes dieser Messreihen zeigt Abbildung 4. 


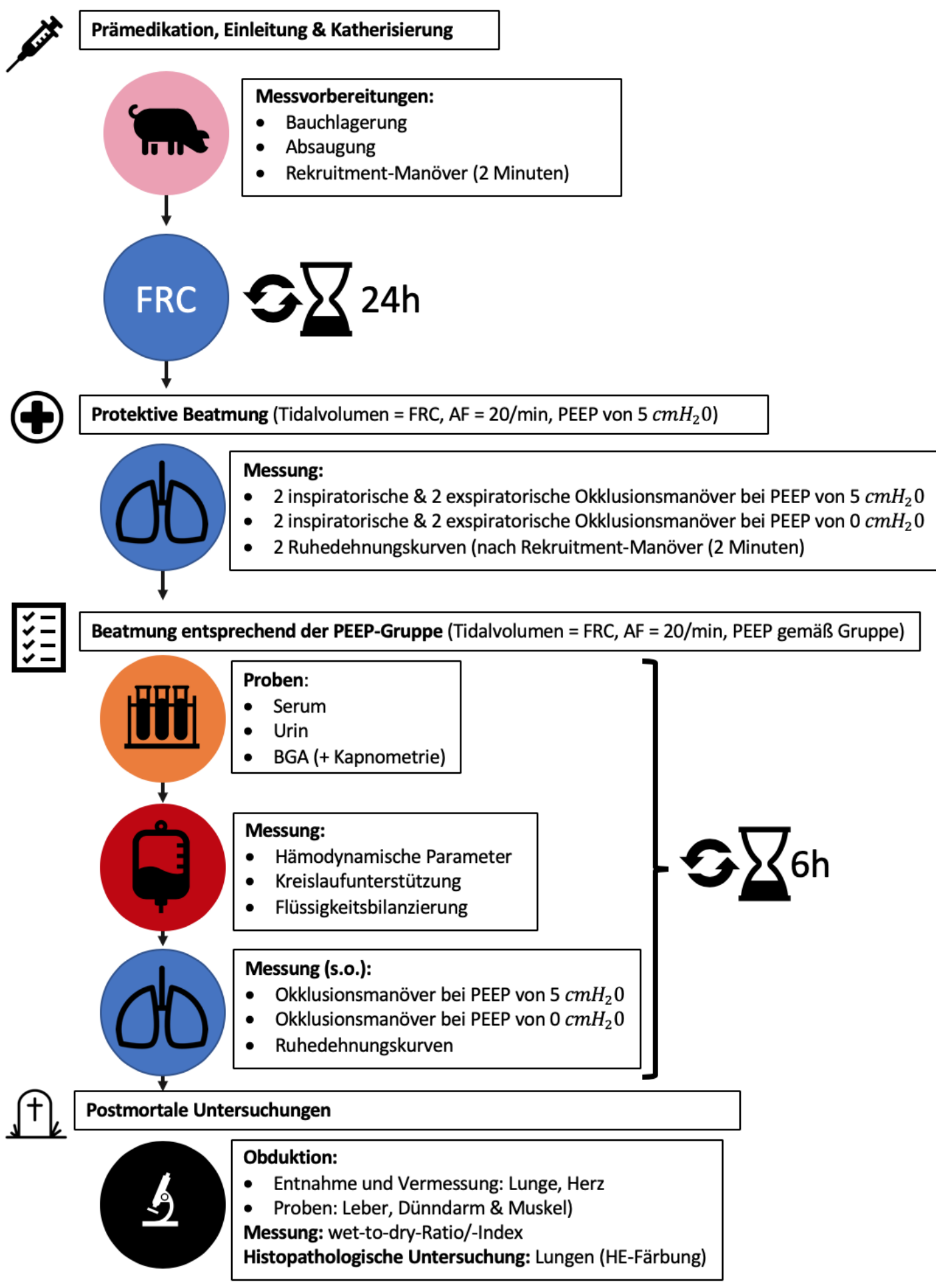

\section{Abbildung 4: Ablauf der Untersuchungen}

Die Abbildung zeigt die Reihenfolge der Untersuchungen. Zunächst wurden die (Mess-)Vorbereitungen abgeschlossen. Anschließend wurde die FRC gemessen. Während einer protektiven Beatmung erfolgten die ersten lungenmechanischen Messungen (blaues Symbol). Sobald das Schwein entsprechend seiner PEEP-Gruppe beatmet wurde, wurden die entsprechenden Parameter (oranges Symbol: Probenentnahme, rotes Symbol: Hämodynamische Messungen, blaues Symbol: Lungenmechanik) gemessen. Anschließend erfolgte die Obduktion mit pathologischer Untersuchung. 


\section{6 Übersicht der erhobenen und berechneten Parameter}

\subsubsection{Atemmechanische Parameter}

Neben der anfänglich bestimmten FRC wurden zusätzlich Standardventilationsparameter wie Tidalvolumen, I:E-Verhältnis, Atemfrequenz, Inspirationszeit, Inspirationspause, $\mathrm{FiO}_{2}$ sowie etC $\mathrm{O}_{2}$ registriert.

Die respiratorischen Drücke wurden aus der Software DIAdem (National Instruments) abgelesen: Der inspiratorische Spitzendruck $\left(P_{P e a k}\right)$, der Beatmungsmitteldruck $\left(P_{A W, \text { mean }}\right)$, die Drücke zu Beginn der Plateauphase $\left(P 1_{r s}\right)$ und Ende der Plateauphase $\left(P 2_{r s}\right)$ in den Atemwegen und im Ösophagus $\left(P 1_{e s} \& P 2_{e s}\right)$, die Drücke in den Atemwegen und im Ösophagus bei appliziertem PEEP (PEEP \& PEEP $P_{e s}$ ) sowie keinem PEEP (Zero PEEP $(Z E E P))\left(Z E E P \& Z E E P_{e s}\right)$. Mithilfe dieser Parameter konnte so jeweils der mittlere Plateaudruck im respiratorischen System $\left(P_{\text {Plateau }}\right)$ und im Ösophagus $\left(P_{\text {Plateau }}\right)$ berechnet werden, um so näherungsweise die treibende Druckdifferenz des respiratorischen Systems $\left(P_{D P, r S}\right)$, den transpulmonaler Inspirationsdruck $\left(P_{t p_{i n s p}}\right)$, den transpulmonaler Exspirationsdruck $\left(P_{t p_{\text {exp }}}\right)$ und die transpulmonale treibende Druckdifferenz $\left(P_{D P, T P}\right)$ zu bestimmen.

$$
\begin{gathered}
P_{t p_{\text {insp }}}=\frac{P_{\text {Plateau }}-P_{\text {Plateau }_{e s}}}{0-Z E E P_{e s}} \\
P_{t p_{\text {exp }}}=\frac{P E E P-0}{P E E P_{e s}-Z E E P_{e s}} \\
P_{D P, T P}=\frac{P_{\text {Plateau }}-P_{\text {Plateau }_{e s}}}{P E E P-P E E P_{e s}} \\
P_{D P, \text { rs }}=P_{\text {Plateau }}-\text { PEEP }
\end{gathered}
$$

Außerdem konnten die folgenden Volumina bestimmt werden: Das PEEP-bedingte Volumen, welches als entweichendes Volumen erfasst wurde, wenn der PEEP auf ZEEP-Niveau gestellt wurde $\left(V_{P E E P}\right)$. Dadurch wurde das end-exspiratorische Volumen (EELV) als Summe aus FRC und $V_{P E E P}$ bestimmt.

$$
E E L V=F R C+V_{P E E P}
$$


Des Weiteren wurde das Volumen ermittelt, welches zwischen Beginn und Ende des Plateaus freigesetzt wurde $\left(V_{\text {Plateau }}\right)$, indem die Volumenänderung während eines inspiratorischen Okklusionsmanöver quantifiziert wurde. Mithilfe dieser bestimmten Parameter können jeweils separat für die Thoraxwand, die Lunge und das gesamte respiratorische System die statische Compliance $\left(C_{r s} \& C_{L}\right)$ und Elastance $\left(E_{r s} \& E_{L}\right)$ berechnet werden.

$$
\begin{gathered}
C_{r s}=\frac{\text { Tidalvolumen }}{P_{\text {Plateau }}-P E E P} \\
E_{r s}=\frac{1}{C_{r s}} \\
C_{L}=\frac{\text { Tidalvolumen }}{P_{t p_{\text {insp }}}-P_{t p_{\text {exp }}}} \\
E_{L}=\frac{1}{C_{L}}
\end{gathered}
$$

Zudem wurden der inspiratorische Fluss und der Atemwegswiderstand $\left(R_{a w}\right)$ berechnet.

$$
R_{a w}=\frac{P_{\text {Peak }}-P_{\text {Plateau }}}{F}
$$

Mithilfe dieser Beatmungsparameter konnte die mechanische Leistung für das respiratorische System bestimmt werden.

$$
\text { Power }_{r s}=0,098 \cdot R R \cdot\left\{\Delta V^{2} \cdot\left[\frac{1}{2} \cdot E_{r s}+R R \cdot \frac{(1+I: E)}{60 \cdot I: E} \cdot R_{a w}\right]+\Delta V \cdot P E E P\right\}
$$

Der stress der Lunge wurde berechnet, indem $P_{\text {Plateau }}$ mit dem Verhältnis von LungenElastance und totaler Elastance multipliziert wurde.

$$
\text { stress }=P_{\text {Plateau }} \cdot \frac{E_{L}}{E_{\text {Total }}}
$$

Die Summe aus Tidalvolumen und appliziertem PEEP-Volumen dividiert durch die FRC, ergab den auf die Lunge wirkenden strain.

$$
\text { strain }=\frac{\text { Tidalvolumen }+V_{P E E P}}{F R C}
$$

Die spezifische Lungenelastance $\left(E_{L_{\text {spec }}}\right)$ wurde ermittelt, indem die Elastance der Lunge $\left(E_{L}\right)$ mit der FRC multipliziert wurde.

$$
E_{L_{\text {spec }}}=E_{L} \cdot F R C
$$


Der in Bezug auf die Entwicklung einer VILI entscheidende Druck ist der transpulmonale Druck (Gattinoni et al. 2017b; Collino et al. 2019). Um die Auswirkungen der applizierten mechanischen Leistung auf das Lungengewebe näher zu untersuchen, müssen die einzelnen Druckkomponenten der bereits vorgestellten Leistungsgleichung des respiratorischen Systems entsprechend modifiziert werden. Die drei Leistungskomponenten (dynamisch, resistiv, statisch) werden im Folgenden theoretisch plausibilisiert (Collino et al. 2019) und durch die experimentellen Ergebnisse wie folgt berechnet:

Erstens die dynamische Komponente der applizierten Leistung ergibt sich durch die treibende Druckdifferenz und die elastischen Rückstellkräfte der Lunge, welche durch die Elastance der Lunge erfasst werden. Daraus ergibt sich für die auf die Lunge wirkende dynamische Druckkomponente $\left(\Delta P_{L, d y n}\right)$ :

$$
E_{L}=\frac{\Delta P_{L, d y n}}{\Delta V}=\frac{P_{\text {plat }}-P E E P}{\Delta V} \Leftrightarrow \Delta P_{L, \text { dyn }}=E_{L} \cdot \Delta V
$$

Zweitens die resistive Komponente der mechanischen Leistung ist durch den Druck zur Überwindung von Widerständen bedingt. Da in den Alveolen hier kaum relevante Strömungswiderstände überwunden werden müssen, liegt es hier nahe, in Bezug auf den transpulmonalen Druck, Gewebewiderstände des Lungengewebes $\left(R_{T}\right)$ bei der Ausdehnung zu betrachten (Collino et al. 2019). Also die Druckänderung bei einem gegebenen (konstantem) Inspirationsfluss $(F)$ z.B. während der Plateau-Phase. Für den dynamischen Widerstand im respiratorischen System ist für die Berechnung des Parallelogramms die Differenz aus Spitzendruck und Plateaudruck bei einem konstanten Fluss benötigt. Der Spitzend ruck herrscht jedoch hauptsächlich in den oberen Atemwegen, sodass für den Bereich der Alveolen der Druckabfall des Plateaudrucks zwischen Beginn der Plateauphase $\left(P 1_{L}\right)$ und Ende der Plateauphase $\left(P 2_{L}\right)$ benötigt wird, um die entscheidende Druckdifferenz zur Überwindung der Lungen-Gewebe-Widerstände $\left(P 1_{L}-P 2_{L}\right)$ zu bestimmen (Protti et al. 2016). Diese Druckdifferenz wird definiert als stress relaxation, welche die beobachtbare Abnahme des stress bei gleichförmigem strain (durch konstanten Fluss) auf die Lungenstruktur ergibt (Cressoni et al. 2016) (s. Abb. 2). Die stress relaxation stellt folglich ein Maß für den Gewebewiderstand des Lungengewebes $\left(R_{T}\right)$ dar $\left(P 1_{L}-P 2_{L}=\right.$ stress relaxation $)$ :

$$
R_{T}=\frac{\Delta P_{L, \text { res }}}{F}=\frac{P 1_{L}-P 2_{L}}{F}=\frac{\text { stress relaxation }}{F} \Leftrightarrow \Delta P_{L, \text { res }}=P 1_{L}-P 2_{L}=R_{T} \cdot F
$$

Dadurch lässt sich die auf die Lunge wirkende widerstandsbedingte Druckkomponente $\left(\Delta P_{L, \text { res }}\right)$ ermitteln. 
Drittens bei einem eventuell vorhandenem PEEP ergibt sich die transpulmonale Komponente, indem man die PEEP-Differenz in den Atemwegen $\left(\triangle P_{a w_{P E E P}}=P E E P-Z E E P\right)$ von der PEEP-Differenz im Pleuraspalt subtrahiert. Mithilfe der PEEP-Differenz im Ösophagus $\left(\Delta P_{e S_{P E E P}}=P_{e S_{P E E P}}-P_{e S_{Z E E P}}\right)$ erhält man einen sehr guten und einfacher zu bestimmenden Näherungswert für die PEEP-Differenz im Pleuraspalt, sodass für die dritte transpulmonale PEEP-bedingte Druckkomponente $\left(\triangle P_{L, P E E P}, P E E P_{t p}\right)$ gilt:

$$
\Delta P_{L, P E E P}=P E E P_{t p} \approx \Delta P_{a w_{P E E P}}-\Delta P_{e S_{P E E P}}=(P E E P-Z E E P)-\left(P E E P_{e s}-Z E E P_{e s}\right)
$$

Bezüglich des gesamten transpulmonalen Drucks $\left(P_{t p}\right)$ ergibt sich daher:

$$
P_{t p}=\Delta P_{L, d y n}+\Delta P_{L, r e s}+\Delta P_{L, P E E P}=E_{L} \cdot \Delta V+R_{T} \cdot F+P E E P_{t p}
$$

Die Energie auf die Lunge pro Atemzug $\left(E_{L, \text { breath }}\right)$ ergibt sich daher mittels Multiplikation mit dem Tidalvolumen:

$$
E_{L, \text { breath }}=\Delta V \cdot \Delta V \cdot E_{L} \cdot \frac{1}{2}+\Delta V \cdot R_{T} \cdot F+\Delta V \cdot P E E P_{t p}
$$

Die Leistung, welche direkt auf die Lunge wirkt $\left(\right.$ Power $\left._{L}\right)$, ergibt sich durch Multiplikation mit der Atemfrequenz und dem Anpassungsfaktor (Collino et al. 2019):

$$
\begin{gathered}
\text { Power }_{L}=0,098 \cdot R R \cdot\left\{\Delta V^{2} \cdot \frac{1}{2} E_{L}+\Delta V \cdot\left(P 1_{L}-P 2_{L}\right)+\Delta V \cdot P E E P_{t p}\right\} \\
\text { Power }_{L}=0,098 \cdot R R \cdot\left\{\Delta V^{2} \cdot \frac{E_{L}}{2}+\Delta V \cdot\left(P 1_{L}-P 2_{L}\right)+\Delta V \cdot\left[(P E E P-Z E E P)-\left(P E E P_{e s}-Z E E P_{e s}\right)\right]\right\}
\end{gathered}
$$

Um eine bessere Vergleichbarkeit zwischen den Versuchstieren zu gewährleisten, wurde die auf die Lunge applizierte mechanische Leistung und die des respiratorischen Systems auf die jeweilige FRC bezogen und als spezifische Leistung $\left(\right.$ Power $_{L_{\text {spec }}}$ und $\left.P o w e r_{r s_{\text {spec }}}\right)$ bezeichnet (Collino et al. 2019):

$$
\begin{aligned}
\text { Power }_{L_{\text {spec }}} & =\frac{\text { Power }_{L}}{F R C} \\
\text { Power }_{r s_{\text {spec }}} & =\frac{\text { Power }_{r s}}{F R C}
\end{aligned}
$$




\subsubsection{Hämodynamische Parameter}

Es wurden aus dem EKG die Herzfrequenz bestimmt und mittels arteriellen Zugangs der systemische arterielle Blutdruck (Systolischer, diastolischer und mittlerer Blutdruck). Mithilfe des Pulmonaliskatheters wurde zudem der pulmonalarterielle Blutdruck (Systolisch, diastolisch und mittlerer Blutdruck) sowie Wedge-Druck und Zentral-venöser Druck (ZVD/CVP) gemessen. Mittels Thermodilutionsmethode wurde das Schlagvolumen und dadurch das Herzzeitvolumen (HZV) bestimmt. Außerdem konnte durch die Thermodilutionsmethode der Gefäßwiderstand im Körper- und Lungenkreislauf (Pulmonalarterieller Widerstand, Systemischer Widerstand) bestimmt werden (Swan et al. 1970). Die gegebenenfalls notwendige Gabe von Kochsalzlösung-/Gelafundin-Infusion sowie der Einsatz von vasoaktiven Substanzen (Noradrenalin) wurden dokumentiert. Die Flüssigkeitsaufnahme wurde durch Addition der Einfuhr durch Infusionen, Perfusoren und eventuelle zusätzliche Flüssigkeitsgaben berechnet. Die Flüssigkeitsausfuhr wird mittels Blasenkatheter und Magensonde bestimmt. Außerdem wurde der Urin gesammelt und das Urinzeitvolumen über den Sammelzeitraum bestimmt. Dadurch wird die Flüssigkeitsbilanz ermittelt.

\subsubsection{Gasaustauschparameter}

Die regelmäßigen Blutgasanalysen von arteriellen, venösen und gemischt-venösen Blutproben zu den Zeitpunkten der Messung der atemmechanischen Größen wurden in Bezug auf $\mathrm{pH}-$ Wert, Basenabweichung (base excess (BE), Sauerstoffpartialdruck $\left(\mathrm{pO}_{2}\right)$, Kohlenstoffdioxidpartialdruck $\left(\mathrm{pCO}_{2}\right)$, endtidales Kohlenstoffdioxid (etCO $\left.\mathrm{C}_{2}\right)$, Hämoglobin $(\mathrm{Hb})$, Sauerstoffsättiung des Hämoglobins $\left(\mathrm{Hb}_{\mathrm{O}_{2}}\right)$, Lactat, Natrium $\left(\mathrm{Na}^{+}\right)$, Chlorid $\left(\mathrm{Cl}^{-}\right)$, Kalium $\left(\mathrm{K}^{+}\right)$, Hämatokrit und Sauerstoffkonzentrationen (arteriell, venös, zentral-venös) untersucht. Im AVEA-Ventilator wurden dann aus den arteriellen Werten für $p \mathrm{O}_{2}$ und $p C \mathrm{O}_{2}$ folgende Parameter berechnet: Kohlenstoffdioxidabgabe $\left(\mathrm{VCO}_{2}\right)$, anatomischer und physiologischer Totraum (als Anteil des Atemzeitvolumens). Ebenfalls wurde das Verhältnis von Sauerstoffpartialdruck und Sauerstoffkonzentration $\left(\mathrm{PaO}_{2} / \mathrm{FiO}_{2}\right)$ sowie die Rechts-Links-Shunt-Fraktion $(R-L-S h u n t)$. Die Shunt-Fraktion ist der prozentuale Anteil des Blutes der zwar vom Herzen ausgeworfen wurde, allerdings nicht zuvor durch die Lungen oxygeniert wurde. Mithilfe des bestimmten $\mathrm{O}_{2}$-Gehaltes in arteriellem $\left(C_{O_{2} \text { art }}\right)$, lungenkapillärem $\left(C_{O_{2} \text { cap }}\right)$ und gemischt-venösen $\left(C_{O_{2 m v}}\right)$ Blut ergibt sich die Shunt-Fraktion durch folgende Formel (Collino et al. 2019): 


$$
R-L-\text { Shunt }=\frac{C_{O_{2_{c a p}}}-C_{O_{2_{a r t}}}}{C_{O_{2_{c a p}}}-C_{O_{2_{m} v}}}
$$

\subsubsection{Postmortale Untersuchungen}

Die folgende Obduktion umfasste die Eröffnung der Cavitas thoracis und der Cavitas abdominalis und die Präparation des Herzes und der Lungen. Nach der Entnahme der Organe, erfolgte die Bestimmung des Lungen- und Herzgewichtes. Anschließend wurden drei Proben (etwa $1 \mathrm{~cm}^{3}$ ) aus jeder Lunge (Ober-, Mittel- und Unterlappen) entnommen und unmittelbar gewogen und nach $24 \mathrm{~h}$ bei $50^{\circ} \mathrm{C}$ wurden die Proben erneut gewogen und das Verhältnis bestimmt (Wet-to-dry-ratio (W/D-Ratio)). Zudem definierten wir einen W/D-Index als W/DRatio multipliziert mit $\frac{H 1}{50 h}$, wobei $H 1$ die tatsächliche Experimentdauer des individuellen Schweins darstellt. Durch diese Indexbildung berücksichtigen wir, dass bei vorzeitig verstorbenen Tieren die mögliche Zeit zur Ödembildung verkürzt war. Dabei nahm dieser Index einen linearen Zusammenhang von Ödembildung und zeitlichem Verlauf an (Collino et al. 2019). Bei den Schweinen 18 - 40 wurden außerdem Proben von Leber, Niere, Dünndarm und Skelettmuskel für eine Bestimmung der Wet-to-dry-Ratio verwendet.

Die Lungen wurden in $4 \%$ Formalin für mindestens 2 Tage fixiert. Zehn Proben von Lungengewebe wurden aus jeder Lunge entnommen. Die Proben wurden fixiert, mittels Alkohol-Reihen dehydriert, mit Xylene gereinigt und in Paraffin eingebettet. Zwei $\mu \mathrm{m}$-Schnitte wurden mittels Mikrotoms geschnitten, auf Trägern aufgetragen und mittels HämatoxylinEosin (HE) gefärbt für die histologische Analyse. Die Analyse wurde verblindet von einer Pathologin durchgeführt. Es wurde ein Score erhoben, der jedem der untersuchten Parameter einen Wert zwischen 0 (nicht vorhanden) bis 100\% (erhebliches Ausmaß) zugeteilt wurde. Dabei wurden die Schnitte auf folgende Läsionsarten untersucht: Gefäßstauungen, Entzündungsreaktionen, Alveolarkollapse/Atelektasenbildungen, Rupturen/septale Erweiterungen, Emphysemen, perivaskulären Ödemen, alveolären Ödemen, alveolären Blutungen und intravaskulären Thromben. Das jeweilige Auftreten dieser Läsionen wurde quantifiziert, indem die Resultate der 20 Schnitte gesammelt und gemeinsam analysiert wurden. Dabei wurde der Anteil der jeweiligen Läsionsart am optischen Gesamtbild (der 20 Schnittproben) erhoben. 


\subsection{Statistische Auswertung}

In Bezug auf das Skalenniveau konnte von einer stetigen Skala ausgehen, da die Merkmale gemessen wurden. Folglich wurden (arithmetische) Mittelwerte und Standardabweichungen bestimmt und als Lage- und Streumaße verwendet und dargestellt, sofern nicht anders gekennzeichnet. Die ermitteln Daten wurden in gruppierten Säulendiagrammen und Streudiagrammen visualisiert. Zur Erfassung von Ereigniszeitdaten wurden Kaplan-Meier-Kurven verwendet.

Es wurden keine A-priori-Berechnungen der statischen Power durchgeführt um die Stichprobengröße zu bestimmen, sondern auf Erfahrungswerte aus vorherigen ähnlichen Experimenten zurückgegriffen (Cressoni et al. 2016; Protti et al. 2015; Protti et al. 2011). Die Anzahl der Versuchstiere entsprach oder übertraf dem Stichprobenumfang vorheriger ähnlicher Experimentreihen und erschien so sinnvoll. Zur Varianzanalyse wurde folgende Methoden angewendet. Student's t-Test wurde für den Vergleich zwischen „Niedriger PEEP“ und „Hoher PEEP“ in Tabelle 4 herangezogen. Um die stetigen Variablen verschiedener Gruppen zu vergleichen, wurden einseitige ANOVA-Tests (Analysis-of-variance-Test (VarianzanalyseTest)) verwendet. Einseitige ANOVA-Tests wurden genutzt, um die Unterschiede zwischen Baseline und Ende des Experimentes zu analysieren und um verschiedene PEEP-Gruppen miteinander zu vergleichen. Um Unterschiede zwischen den PEEP-Gruppen im zeitlichen Verlauf zu erfassen, wurde ein lineares gemischtes Modell (linear mixed effects mode) verwendet, wobei PEEP-Gruppe, Zeit in Stunden und deren Interaktion als fixe Effekte und die Versuchstiere als Zufallsvariable aufgefasst wurden. Dadurch wurden nicht vorhandene Datenpunkte ignoriert, obwohl letztlich die Aussagekraft der Originalstichprobengröße erhalten blieb. Korrelationsanalysen wurden mithilfe der Pearson-Korrelation berechnet. KaplanMeier-Kurven und log-rank-Tests wurden zur Analyse der Mortalität im zeitlichen Verlauf genutzt. Ein p-Wert $<0,05$ wurde dabei als statistisch signifikant gewertet. Es wurden keine Ausreißer aus der weiteren Analyse ausgeschlossen. Um einen möglichen „schädlichen“ Leistungsgrenzwert festzulegen, wurde im Rahmen des Projektes der Arbeitsgruppe mehrere receiver operating characteristic (ROC-)Kurven erstellt, wobei der Median diverser Variablen als cutoff-Wert benutzt wurde. Eine logistische Regression wurde verwendet um diejenigen Variablen zu identifizieren, welche mit dem Grenzwert für die mechanische Leistung zusammenhängen. Für nähere Details dazu sei auf die Veröffentlichung der Arbeitsgruppe verwiesen (Collino et al. 2019). Die Analysen wurden mithilfe von „R software“ durchgeführt (R Project for Statistical Computing, http://www.r-project.org/). 


\section{Ergebnisse}

\subsection{Allgemeine Ergebnisse zu den Versuchstieren}

Für die Versuche wurden 40 weibliche durchschnittlich 4 Monate alte Hausschweine verwendet. Zwei von diesen Schweinen wurden auf Grund von Septitiden in Folge einer Blasenfehlpunktion nicht in die weiteren Betrachtungen eingeschlossen. Zwei weitere Schweine starben vor dem Beginn der Messreihen und wurden daher ebenfalls aus der weiteren Analyse ausgeschlossen. Die verbleibenden 36 Schweine wiesen initial ein Durchschnittsgewicht von 23,3 $\pm 2,3 \mathrm{~kg}$ und am Ende ein Gewicht von 25,6 \pm 2,7 $\mathrm{kg}$ bei einer durchschnittlichen Länge von der Nasenspitze bis zum Schwanzansatz von 91,8 \pm 5,2 cm und einem durchschnittlichen Thoraxumfang von 59,0 $\pm 2,8 \mathrm{~cm}$ auf. 


\subsection{PEEP und mechanische Leistung}

Alle Schweine wurden kontinuierlich mit einer Beatmungsfrequenz von $30 \frac{1}{\min }$, einer $\mathrm{FiO}_{2}$ von 0,4 und einem I:E-Verhältnis von 1:2 ventiliert. Das mittlere Tidalvolumen von 14,9 \pm $2,5 \frac{\mathrm{ml}}{\mathrm{kg} \text { Körpergewicht }}$ ergab sich durch die initial bestimmten FRC. Dadurch wurde ein dynamischer strain von 1 erreicht. Die mechanische Leistung wurde für die Versuchstiere der sechs verschiedenen PEEP-Gruppen zu den jeweiligen Messzeitpunkten aus den respiratorischen Parametern berechnet. Abb. 5 zeigt die mechanische Leistung, welche auf das respiratorische System wirkte und diejenige Leistung, welche nur auf die Lunge wirkte als Funktion des am Beatmungsgerät gewählten PEEP gemittelt über die gesamte Dauer des Experiments. Dabei beinhaltet jede der gestapelten Säulen die verschiedenen Leistungskomponenten (statisch, dynamisch, resistiv). Es zeigte sich, dass die applizierten mechanische Leistungen in den niedrigeren PEEP-Bereichen (PEEP $0 \mathrm{~cm} \mathrm{H}_{2} 0: 8,8 \pm 3,8 \frac{\mathrm{J}}{\mathrm{min}}$, PEEP $4 \mathrm{~cm} \mathrm{H}_{2} 0$ : $8,9 \pm 4,4 \frac{\mathrm{J}}{\min }$ und PEEP $\left.7 \mathrm{~cm} \mathrm{H}_{2} 0: 9,6 \pm 4,3 \frac{\mathrm{J}}{\mathrm{min}}\right)$ relativ ähnlich waren $(\mathrm{p}=0,513)$, wobei die PEEP-bedingte Zunahme der statischen Komponente durch eine zeitgleiche Reduktion der dynamischen und der resistiven Komponente einherging. Dahingegen folgte einem weiteren Anstieg des PEEP auf höhere Werte (PEEP $11 \mathrm{~cm} \mathrm{H}_{2} 0: 15,5 \pm 3,6 \frac{\mathrm{J}}{\mathrm{min}}$, PEEP $14 \mathrm{~cm} \mathrm{H}_{2} 0: 18,7 \pm 6 \frac{\mathrm{J}}{\min }$ und PEEP $\left.18 \mathrm{cmH}_{2} 0: 22 \pm 6,1 \frac{\mathrm{J}}{\min }\right)$ jeweils ein proportionaler Anstieg der mechanischen Leistung, hauptsächlich bedingt durch die statische Komponente, da die dynamische und resistive Komponente unverändert blieben. Die dynamische Komponente der mechanischen Leistung (bedingt durch die treibende Druckdifferenz) nahm signifikant von ZEEP zu PEEP-Werten von 4 und $7 \mathrm{~cm} \mathrm{H}_{2} \mathrm{O}$ (Gruppe: „Niedriger PEEP“) ab und stieg bei höheren PEEP-Werten von 11, 14 und $18 \mathrm{cmH}_{2} \mathrm{O}$ (Gruppe: „Hoher PEEP“) wieder auf ähnlich hohe Werte wie bei der ZEEP-Gruppe an. Folglich war die dynamische Leistung bei der niedrigen PEEP-Gruppe (ohne ZEEP) am niedrigsten und bei ZEEP und in der Gruppe mit einem hohen PEEP vergleichbar hoch. 

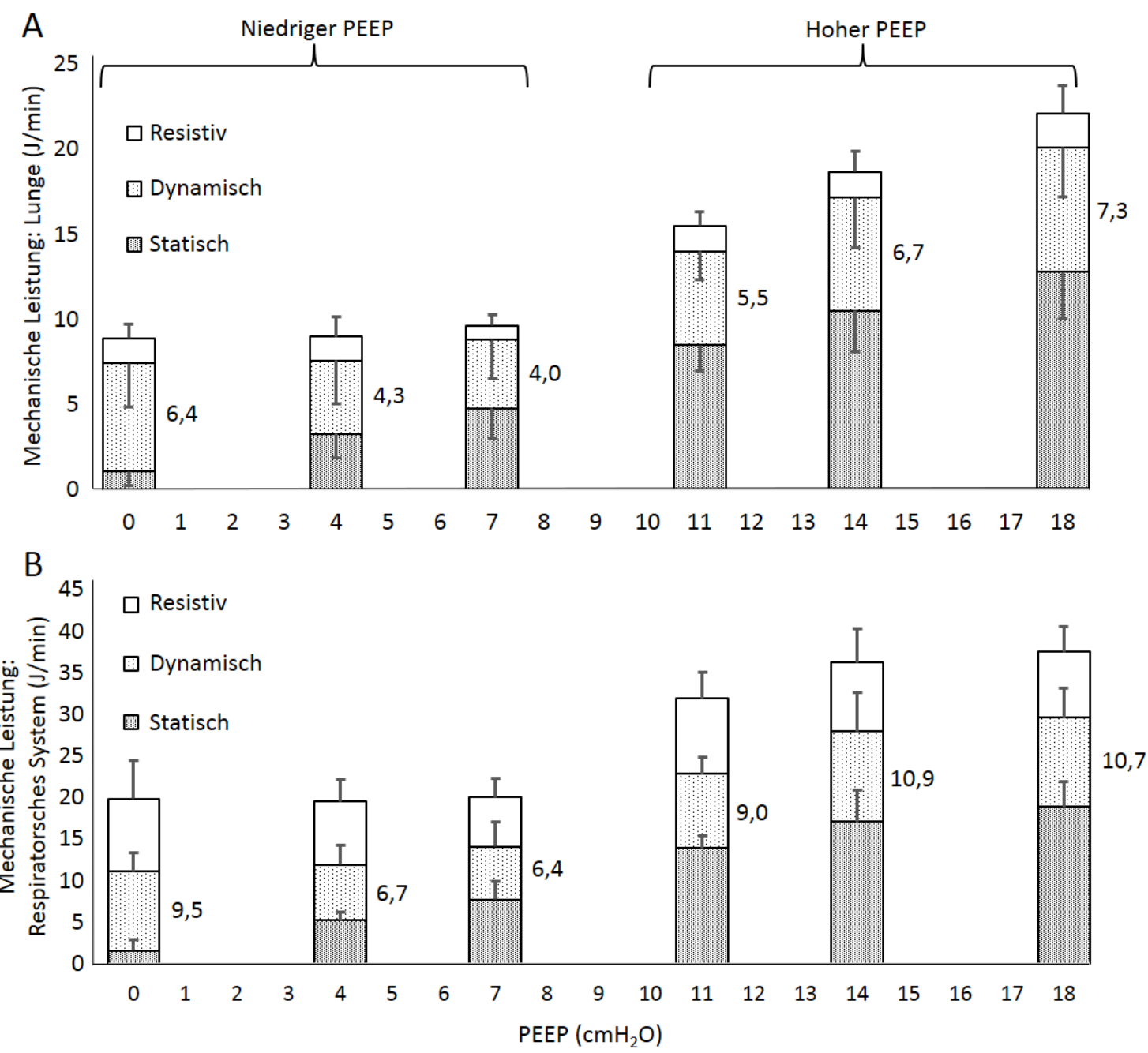

Abbildung 5: Mechanische Leistung: Lunge (A) und respiratorisches System (B) in den PEEP-Gruppen

Die mechanische Leistung gemittelt über die gesamte Dauer des Experiments und deren drei Bestandteile (resistiv (weiß), dynamisch (gepunktet) und statisch (grau) der verschiedenen PEEP-Gruppen werden als gestapelte Säulen dargestellt. Dabei zeigt die obere Abbildung (A) die mechanische Leistung, welche auf die Lunge wirkt und die untere Abbildung (B) diejenige Leistung, welche auf das gesamte respiratorische System wirkte. Um die jeweiligen dynamischen Komponenten besonders hervorzuheben, ist deren Wert neben den gepunkteten Säulen gekennzeichnet. Zur weiteren Betrachtung wurden die PEEP-Gruppen in „Niedriger PEEP“ (0, 4 und $\left.7 \mathrm{cmH}_{2} \mathrm{O}\right)$ und „Hoher PEEP“ (11, 14 und $18 \mathrm{cmH}_{2} \mathrm{O}$ ) eingeteilt. Die Verwendung der Abbildung erfolgt mit freundlicher Genehmigung des Wolters Kluwer Health Verlags (Collino et al. 2019). 


\subsection{Gewählter PEEP und mechanische Leistung im zeitlichen Verlauf}

Die mechanische Leistung, welche auf die Lunge (Abb. 6A) und auf das respiratorische System (Abb. 6B) wirkte, unterschied sich in den verschiedenen PEEP-Gruppen deutlich untereinander und steigt im zeitlichen Verlauf tendenziell (vor allem im Bereich um $24 h-30$ h) an.

Wenn man die mechanische Leistung über die Zeit integriert, erhält man die mechanische Arbeit. In Abb. 6C wird diese mechanische Arbeit im zeitlichen Verlauf dargestellt. Die in Abb. 6A und 6B erwähnte Zunahme erkennt man in einem stärkeren Anstieg. Es wird deutlich, dass sich der größte Unterschied in der mechanischen Arbeit und daher auch in deren Anstieg zwischen den PEEP-Gruppen mit einem PEEP von 0,4 und $7 \mathrm{~cm} \mathrm{H}_{2} \mathrm{O}$ und denen mit einem höheren PEEP (11, 14 und $\left.18 \mathrm{~cm} \mathrm{H}_{2} 0\right)$ zeigt. 
A

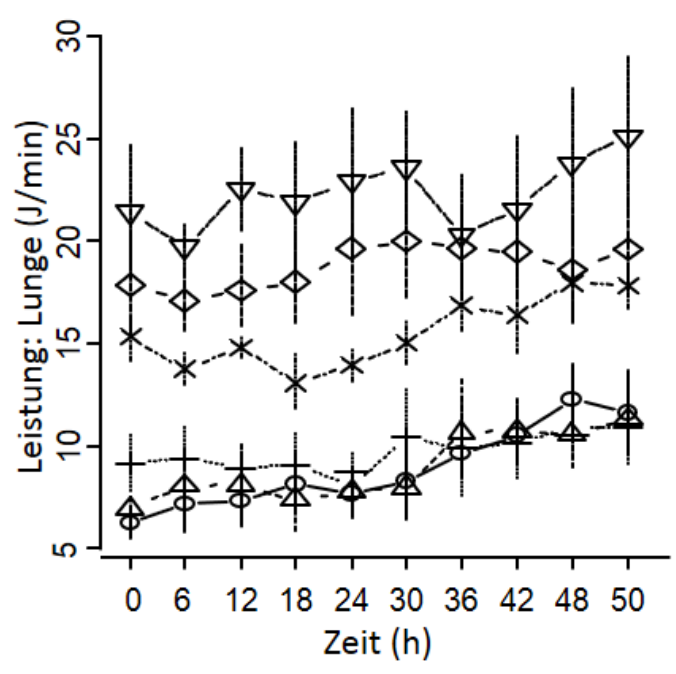

B

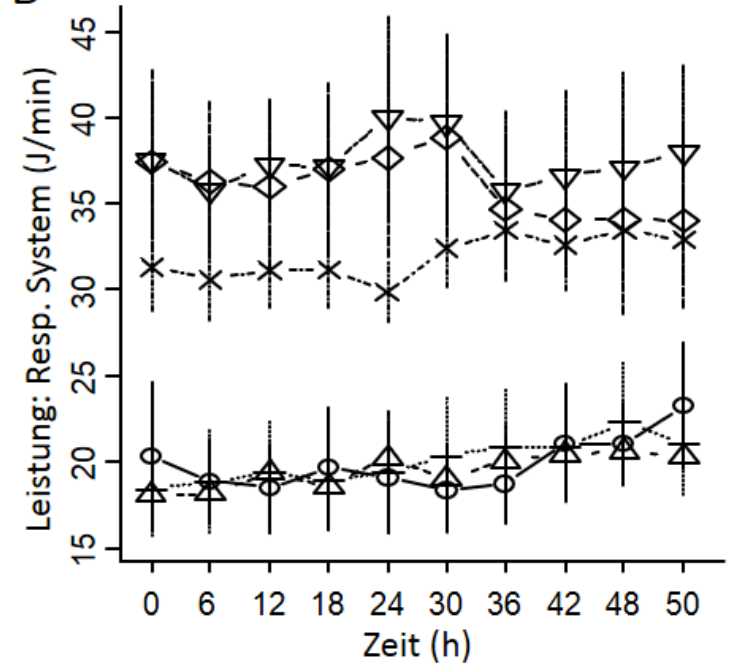

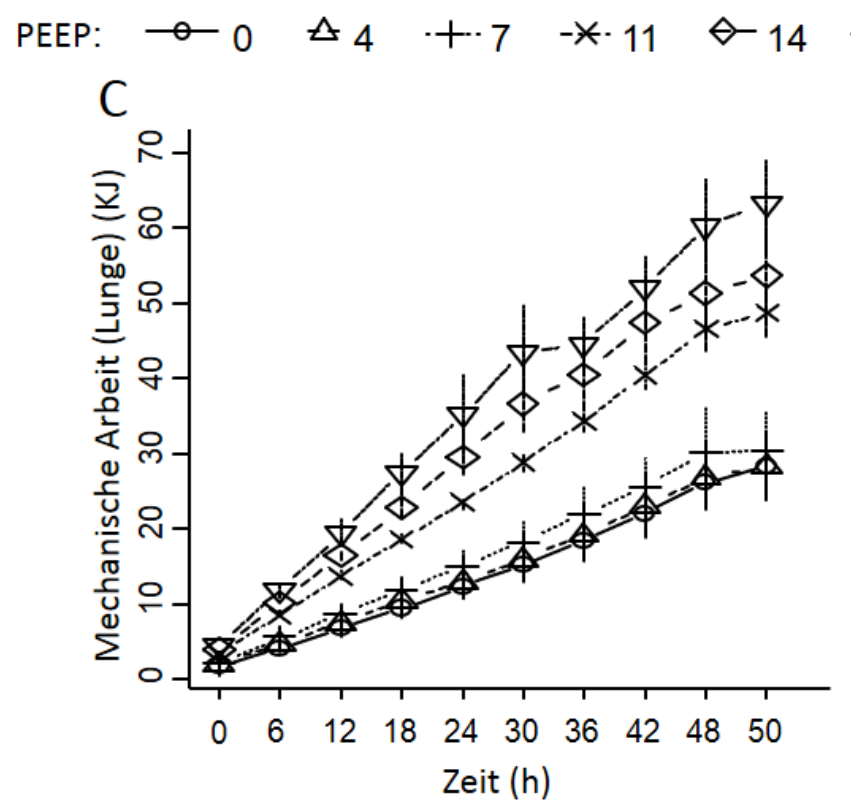

Abbildung 6: Mechanische Leistung und Arbeit im zeitlichen Verlauf

Die mechanische Leistung, welche auf die Lunge (A, Varianzanalyse: Linear gemischtes Modell: Zeit $(p<0,001)$, PEEP $(p<0,001)$, PEEP:Zeit-Interaktion $(p=0,713))$ und auf das respiratorische System (B, Varianzanalyse: Linear gemischtes Modell: Zeit $(p<0,001)$, PEEP $(p<0,001)$, PEEP:ZeitInteraktion $(p=0,696))$ wirkte sich in den verschiedenen PEEP-Gruppen deutlich unterschied und im zeitlichen Verlauf tendenziell anstieg. Dabei wurden die jeweiligen Mittelwerte zusammen mit der Standardabweichung dargestellt. Im unteren Graphen wird die verrichtete mechanische Arbeit der verschiedenen PEEP-Gruppen, welche auf die Lunge wirkte, bis zum jeweiligen Zeitwert aufsummiert und im zeitlichen Verlauf dargestellt (C, Varianzanalyse: Linear gemischtes Modell: Zeit ( $\mathrm{p}$ 0,001), PEEP ( $p<0,001)$, PEEP:Zeit-Interaktion $(p<0,001))$. Die Legende für die PEEP-Werte in dieser und den nachfolgenden Abbildungen bezieht sich auf die jeweilige Gruppe mit den entsprechenden Werten in $\mathrm{cmH}_{2} \mathrm{O}$. Die Verwendung der Abbildung erfolgt mit freundlicher Genehmigung des Wolters Kluwer Health Verlags (Collino et al. 2019). 
Im zeitlichen Verlauf starben fünf der 36 Schweine vorzeitig durch einen Spannungspneumothorax $\left(\mathrm{n}=1\right.$; PEEP $\left.18 \mathrm{cmH}_{2} 0\right)$ oder hämodynamischen Versagen $(\mathrm{n}=2$ : PEEP $14 \mathrm{cmH}_{2} 0 ; \mathrm{n}=2$ : PEEP $\left.18 \mathrm{cmH}_{2} 0\right)$. Die Unterschiede in der Mortalität sind zwischen den verschiedenen PEEP-Gruppen signifikant $(p=0,012)$ (s. Abb. 7).

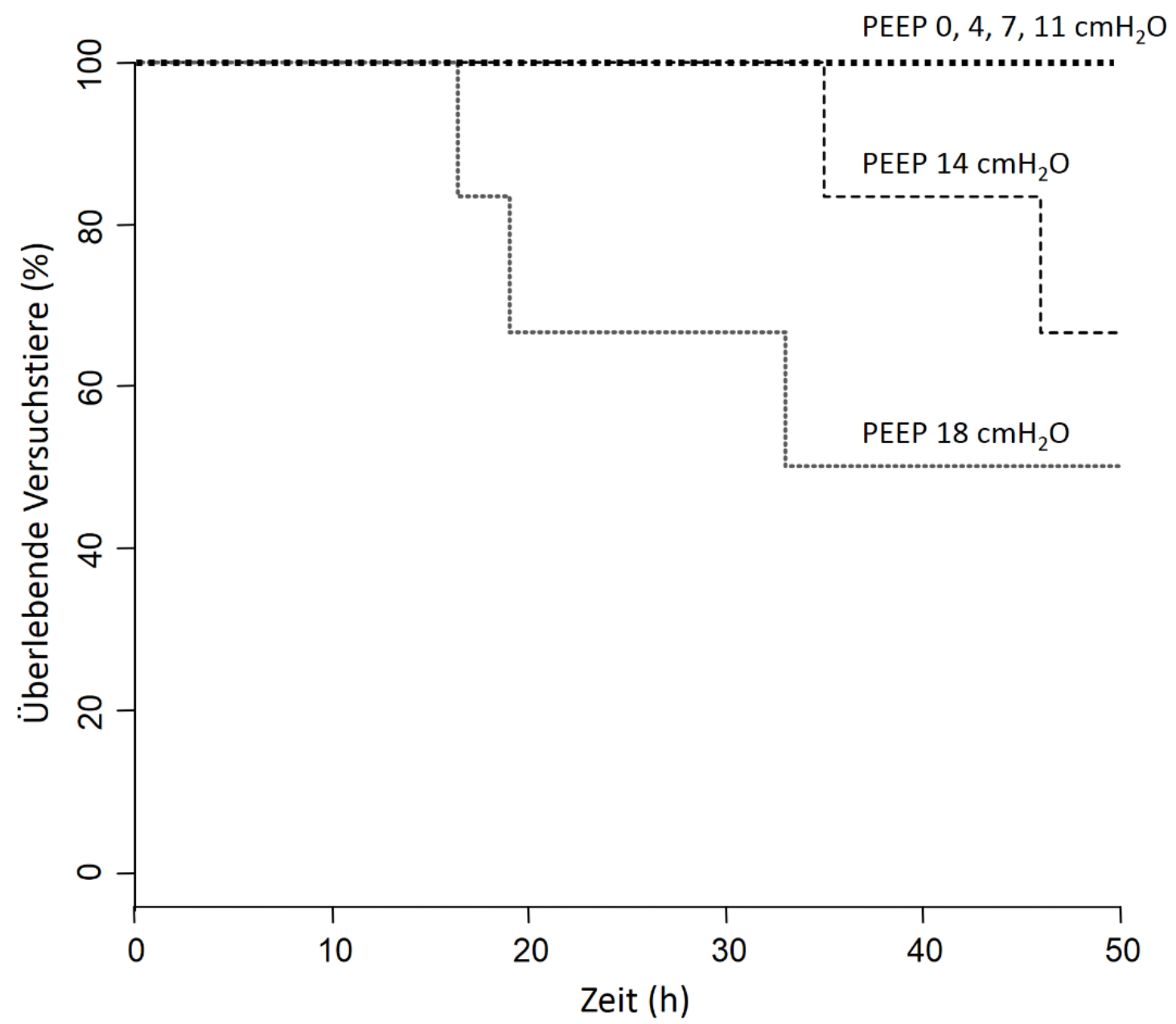

\section{Abbildung 7: Überlebende Versuchstiere im Verlauf der Zeit (Kaplan-Meyer-Kurve)}

In dieser Kaplan-Meyer-Kurve wird das Überleben der Versuchstiere in Abhängigkeit von der Zeit dargestellt. Es starben nur Tiere aus den PEEP-Gruppen mit 14 und $18 \mathrm{cmH}_{2} \mathrm{O}$ (Log-rank Test: $\mathrm{p}=$ 0,012). Die Verwendung der Abbildung erfolgt mit freundlicher Genehmigung des Wolters Kluwer Health Verlags (Collino et al. 2019). 


\subsection{Gewählter PEEP in Bezug zu den lungenmechanischen Eigenschaften}

Abb. 8 zeigt für die verschiedenen PEEP-Gruppen den zeitlichen Verlauf verschiedener lungenmechanischer Einflussgrößen. So wird das jeweilige end-exspiratorische Lungenvolumen (EELV; FRC + PEEP-Volumen) im Verlauf der Zeit präsentiert (Abb. 8A). Hierin erkennt man deutlich den Einfluss des PEEP-Volumens durch den Vergleich der verschiedenen PEEP-Gruppen. Bei diesen jeweiligen Lungenvolumina werden auch andere respiratorischen Parameter wie beispielsweise der Plateaudruck in den Atemwegen (Abb. 8B), die treibende Druckdifferenz in den Atemwegen (Abb. 8C) und die treibende transpulmonale Druckdifferenz (Abb. 8D) erfasst.

Zu Beginn des Experimentes $(0 h)$ sind all diese Drücke mit dem EELV korreliert $(\mathrm{p}<0,001$; Bestimmtheitsmaße: Plateau Atemwegsdruck $r^{2}=0,85$, treibende Druckdifferenz $r^{2}=0,50$, treibende transpulmonale Druckdifferenz $\left.r^{2}=0,24\right)$. Anschließend stiegen diese Drücke im zeitlichen Verlauf tendenziell an (insbesondere bei $24-30 \mathrm{~h}$ ). Beachtenswert ist die Tatsache, dass die größte Verschlechterung der lungenmechanischen Eigenschaften, welche sich in diesem Fall vor allem im Anstieg der notwendigen Beatmungsdrücke zeigt, sowohl für den Atemwegsdruck als auch die transpulmonalen treibenden Druckdifferenzen in der ZEEPGruppe beobachtet werden kann. 
A

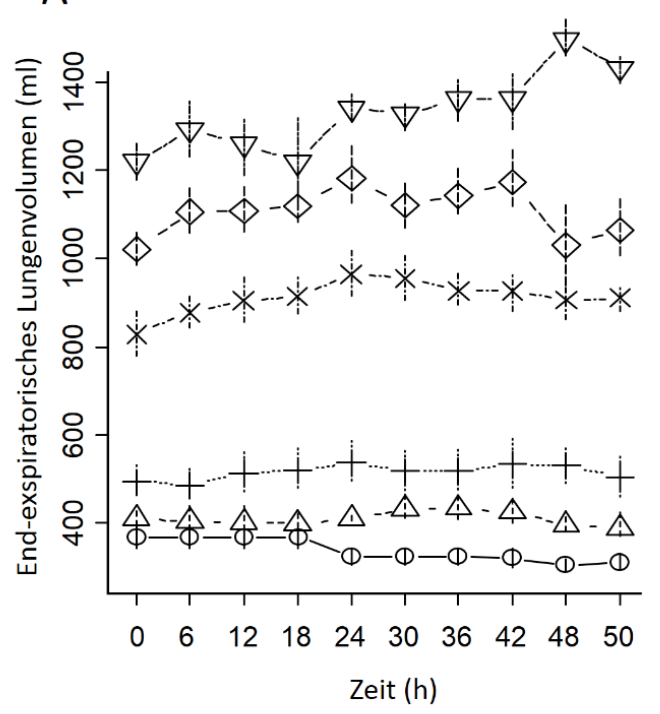

B

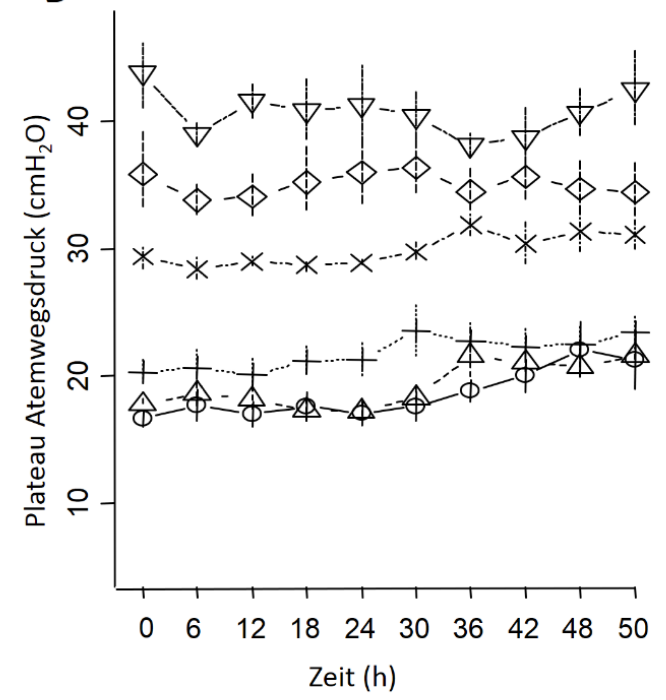

PEEP: $-0 \quad 04 \cdot+\cdot 7 \quad-* 11 \quad \diamond-14 \quad \nabla \cdot 18$

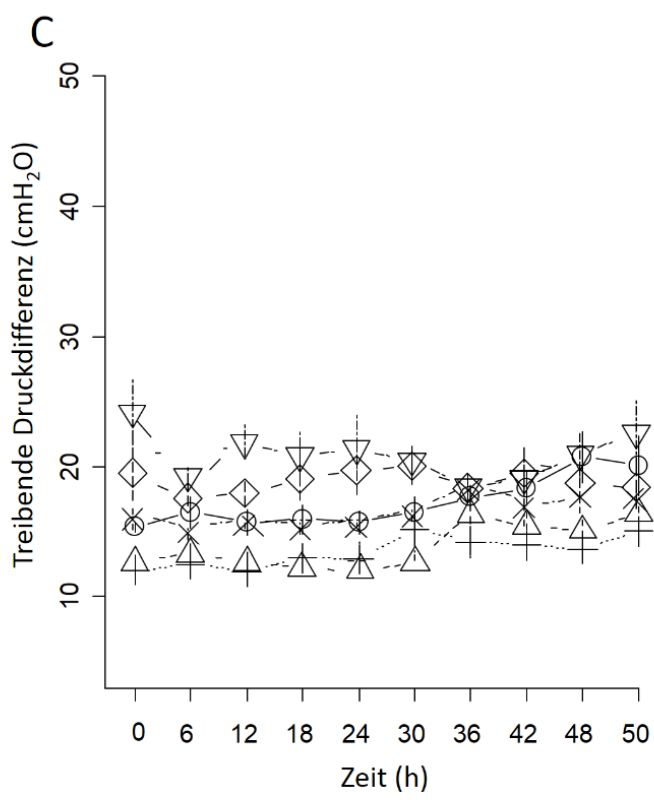

D

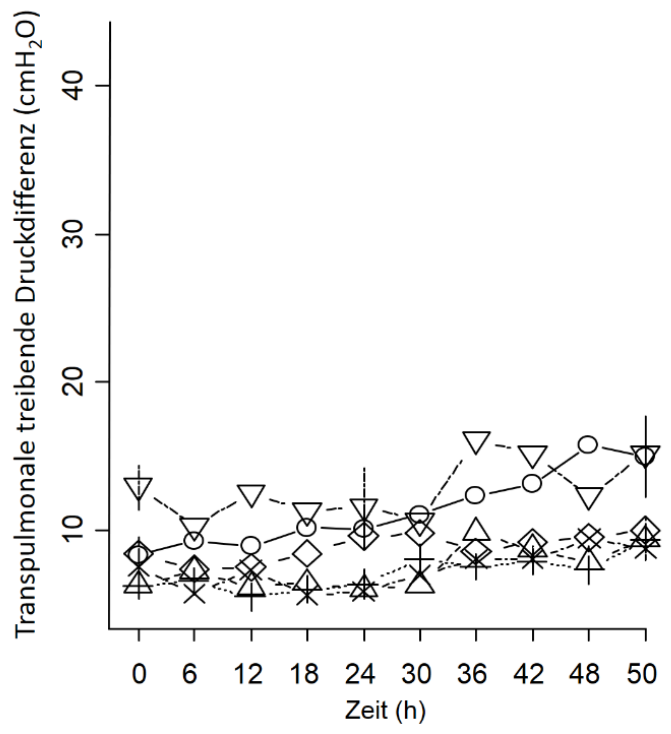

\section{Abbildung 8: Respiratorische Parameter im zeitlichen Verlauf}

Das end-exspiratorische Lungenvolumen (EELV; FRC + PEEP-Volumen) (A, Varianzanalyse: Linear gemischtes Modell: Zeit $(p=0,023)$, PEEP $(p<0,001)$, PEEP:Zeit-Interaktion $(p=0,010))$, den Plateaudruck in den Atemwegen (B, Varianzanalyse: Linear gemischtes Modell: Zeit $(p<0,001)$, PEEP ( $p<0,001)$, PEEP:Zeit-Interaktion $(p=0,413)$ ), die treibende Druckdifferenz in den Atemwegen (C, Varianzanalyse: Linear gemischtes Modell: Zeit ( $p<0,001)$, PEEP ( $p<0,001)$, PEEP:ZeitInteraktion $(p=0,334))$ und die treibende transpulmonale Druckdifferenz $(\mathrm{D}$, Varianzanalyse: Linear gemischtes Modell: Zeit ( $p<0,001)$, PEEP ( $p<0,001)$, PEEP:Zeit-Interaktion $(p=0,427))$ werden für die verschiedenen PEEP-Gruppen im zeitlichen Verlauf dargestellt. Die Verwendung der Abbildung erfolgt mit freundlicher Genehmigung des Wolters Kluwer Health Verlags (Collino et al. 2019). 
In Abb. 9 geht hervor, dass die Lungenelastance im zeitlichen Verlauf in allen PEEP-Gruppen anstieg (s. Abb. 9A). Trotz einer kleinen Abnahme in der Elastance der Thoraxwand (s. Abb. 9B), führte dies dazu, dass die Elastance des gesamten respiratorischen Systems ebenfalls anstieg (s. Abb. 9C). Auch die Elastance-Werte waren zu Beginn des Experiments $(0 h)$ mit dem jeweiligen end-exspiratorischen Lungenvolumen (EELV) korreliert $(p<0,001)$ und die größte Zunahme der Lungenelastance und der damit einhergehenden Verschlechterung der Lungenmechanik konnte in der ZEEP-Gruppe beobachtet werden (von $23 \pm 5$ bis 46 $\left.\pm 2 \mathrm{cmH}_{2} 0 ; \mathrm{p}=0,044\right)$. 


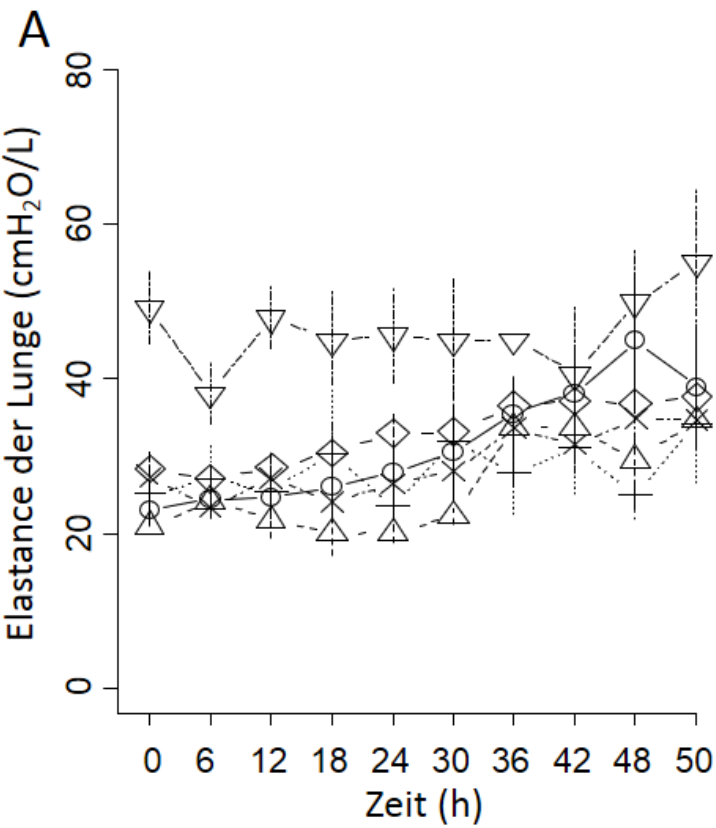

B
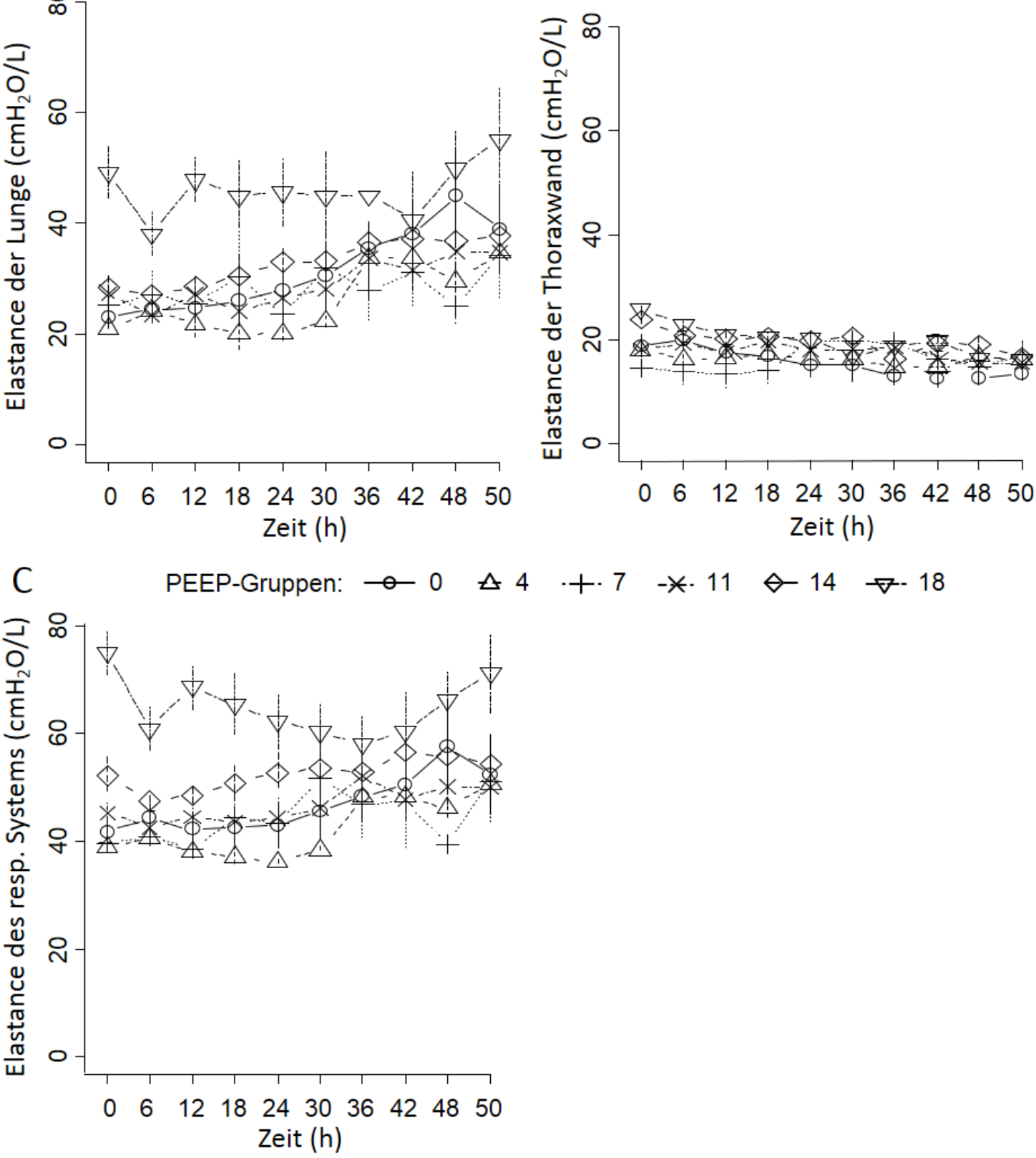

\section{Abbildung 9: Veränderungen der Elastance-Werte im zeitlichen Verlauf}

In der Abbildung wird die Lungenelastance (A, Varianzanalyse: Linear gemischtes Modell: Zeit $(\mathrm{p}<$ 0,001), PEEP $(p<0,001)$, PEEP:Zeit-Interaktion $(p=0,445)$ ), die Elastance der Thoraxwand $(B$, Varianzanalyse: Linear gemischtes Modell: Zeit $(p<0,001)$, PEEP ( $p=0,002)$, PEEP:Zeit-Interaktion $(\mathrm{p}=0,499))$ und die Elastance des gesamten respiratorischen Systems (C, Varianzanalyse: Linear gemischtes Modell: Zeit $(p=0,005)$, PEEP ( $p=0,103)$, PEEP:Zeit-Interaktion $(p=0,125))$ im zeitlichen Verlauf in den verschiedenen PEEP-Gruppen dargestellt. Dabei handelt es sich um die Mittelwerte der jeweiligen Gruppen und die dazugehörige Standardabweichung. Die Verwendung der Abbildung erfolgt mit freundlicher Genehmigung des Wolters Kluwer Health Verlags (Collino et al. 2019). 
Abb. 10 zeigt die Parameter der mechanischen Belastung der Lunge in Form des stress, des gesamten strain und der Faktor diese Werte ineinander umzurechnen, die spezifische LungenElastance (s. Kapitel 1.6.2). Der stress wurde als Produkt aus $P_{\text {Plateau }}$ mit dem Verhältnis von Lungen-Elastance und totaler Elastance berechnet. Die Summe aus Tidalvolumen und appliziertem PEEP-Volumen, dividiert durch die FRC, ergab den auf die Lunge wirkenden strain. Die spezifische Lungenelastance wurde ermittelt, indem die Elastance mit der FRC multipliziert wurde. Der stress zum Startzeitpunkt war umso höher, je größer auch der gewählte PEEP war (s. Abb. 10A). Außerdem zeigte sich die Tendenz, dass der stress im zeitlichen Verlauf anstieg. Der strain änderte sich nicht wesentlich über die Dauer des Experimentes (s. Abb. 10B). Der Anstieg in der Gruppe mit dem PEEP von $18 \mathrm{cmH}_{2} 0$ zwischen 18 und 42 Stunden stellte hierbei eine Ausnahme dar, wobei berücksichtigt werden sollte, dass in diesem Zeitraum drei Versuchstiere dieser Gruppe vorzeitig verstarben (s. Abb. 7). Folglich zeigt die spezifische Lungenelastance als Proportionalitätskonstante zwischen stress und strain, welche die intrinsische Elastizität des Lungengewebes darstellt, einen Anstieg im Laufe der Zeit und damit eine Verschlechterung der mechanischen Lungeneigenschaften in allen PEEP-Gruppen (s. Abb. 10C).

In der Tabellen 1 wird eine Übersicht der respiratorischen Parameter in den verschiedenen PEEP-Gruppen sowohl zu Beginn als auch am Ende des Experimentes dargestellt. 
A

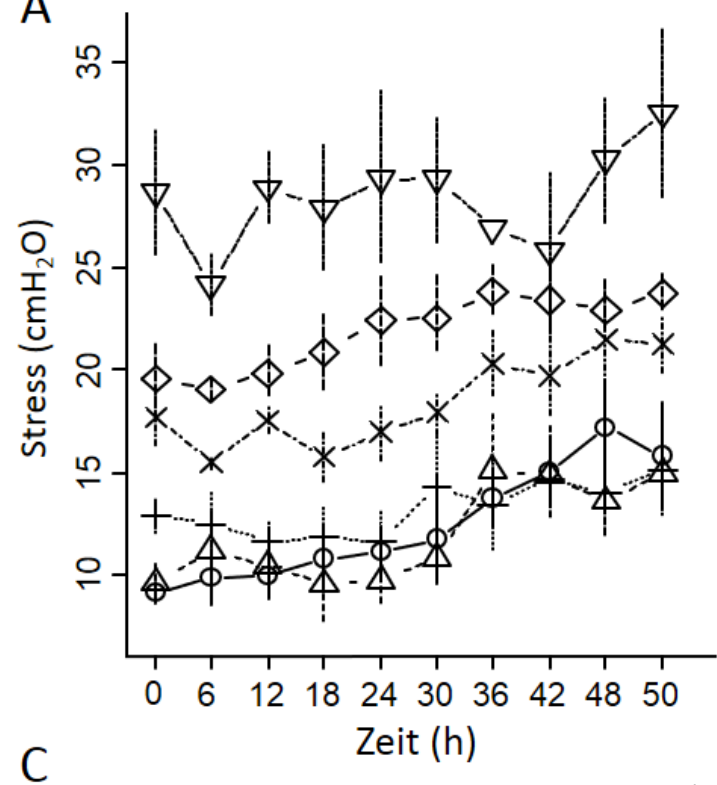

$\mathrm{C}$

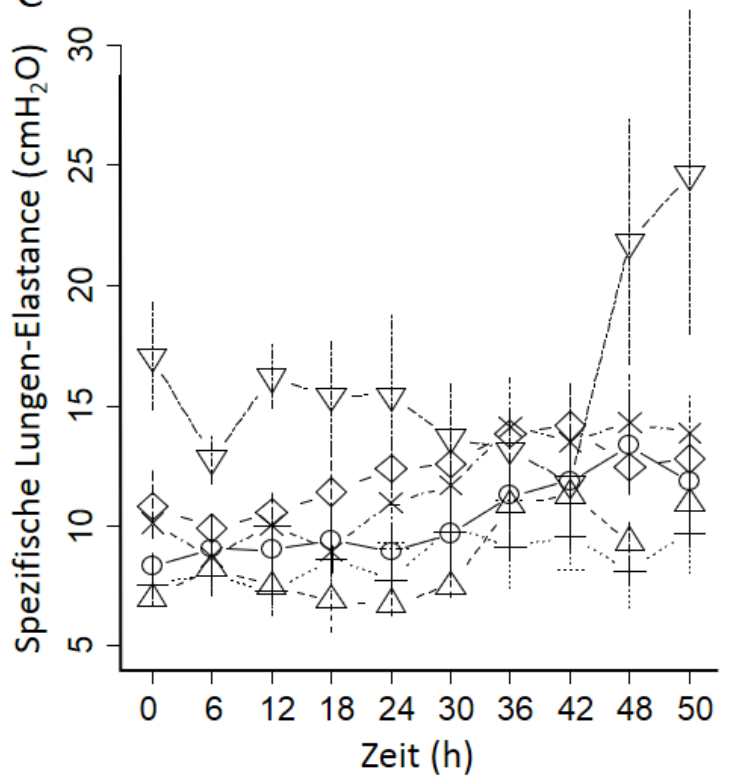

B

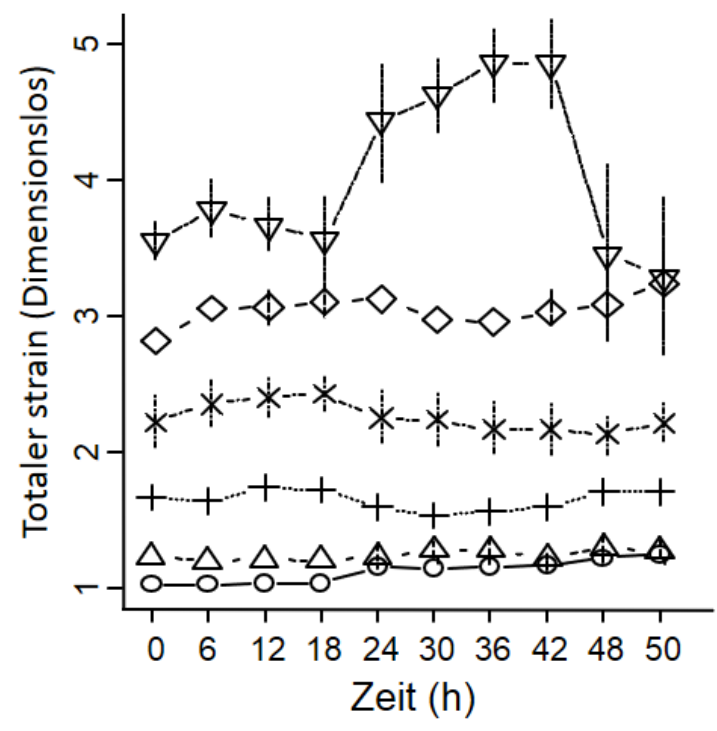

PEEP-Gruppen:

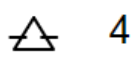

$\cdot+\cdot \cdot 7$

$-\ngtr \cdot 11$

$\diamond-14$

$-\nabla-18$

\section{Abbildung 10: Stress, totaler strain und spezifische Lungenelastance im zeitlichen Verlauf}

In der obigen Grafik wird der auf die Lunge wirkende stress (A, Varianzanalyse: Linear gemischtes Modell: Zeit $(\mathrm{p}<0,001)$, PEEP $(\mathrm{p}<0,001)$, PEEP:Zeit-Interaktion $(\mathrm{p}=0,667))$, der gesamte strain (B, Varianzanalyse: Linear gemischtes Modell: Zeit $(p=0,379)$, PEEP $(p<0,001)$, PEEP:Zeit-Interaktion $(\mathrm{p}<0,001))$ und die spezifische Lungen-Elastance (C, Varianzanalyse: Linear gemischtes Modell: Zeit $(p<0,001)$, PEEP ( $p<0,001)$, PEEP:Zeit-Interaktion $(p=0,032))$ der verschiedenen PEEP-Gruppen im zeitlichen Verlauf dargestellt. Die Verwendung der Abbildung erfolgt mit freundlicher Genehmigung des Wolters Kluwer Health Verlags (Collino et al. 2019). 
Tabelle 1: Respiratorische Parameter

\begin{tabular}{|c|c|c|c|c|c|c|c|c|c|c|}
\hline Gewählter PEEP & $\mathbf{0}$ & 4 & 7 & 11 & 14 & 18 & \multirow{2}{*}{$\begin{array}{c}\text { P- } \\
\text { Wert }\end{array}$} & \multirow{2}{*}{ Zeit } & \multirow{2}{*}{ PEEP } & \multirow{2}{*}{$\begin{array}{l}\text { Zeit:PEEP- } \\
\text { Interaktion }\end{array}$} \\
\hline Anzahl Schweine & 6 & 6 & 6 & 6 & $6^{*}$ & $6^{* * *}$ & & & & \\
\hline \multirow{2}{*}{$\mathbf{V}_{T}(\mathbf{m l})$} & $\begin{array}{c}375 \\
\pm 58\end{array}$ & $\begin{array}{r}325 \\
\pm 45\end{array}$ & $\begin{array}{r}310 \\
\pm 90\end{array}$ & $\begin{array}{c}361 \\
\pm 36\end{array}$ & $\begin{array}{c}372 \\
\pm 69\end{array}$ & $\begin{array}{c}326 \\
\pm 60\end{array}$ & 0.336 & \multirow{2}{*}{0.656} & \multirow{2}{*}{0.330} & \multirow{2}{*}{0.346} \\
\hline & $\begin{array}{l}373 \\
\pm 57\end{array}$ & $\begin{array}{c}325 \\
\pm 40\end{array}$ & $\begin{array}{l}312 \\
\pm 90\end{array}$ & $\begin{array}{r}360 \\
\pm 36\end{array}$ & $\begin{array}{l}374 \\
\pm 75\end{array}$ & $\begin{array}{l}330 \\
\pm 57\end{array}$ & 0.378 & & & \\
\hline \multirow{2}{*}{ FRC (ml) } & $\begin{array}{l}369 \\
\pm 65\end{array}$ & $\begin{array}{r}333 \\
\pm 68\end{array}$ & $\begin{array}{c}305 \\
\pm 86\end{array}$ & $\begin{array}{c}375 \\
\pm 60\end{array}$ & $\begin{array}{l}370 \\
\pm 75\end{array}$ & $\begin{array}{l}342 \\
\pm 47\end{array}$ & 0.451 & \multirow{2}{*}{0.997} & \multirow{2}{*}{$<0.001$} & \multirow{2}{*}{$<0.001$} \\
\hline & $\begin{array}{l}305 \\
\pm 34\end{array}$ & $\begin{array}{r}317 \\
\pm 59\end{array}$ & $\begin{array}{l}302 \\
\pm 72\end{array}$ & $\begin{array}{r}399 \\
\pm 38\end{array}$ & $\begin{array}{l}356 \\
\pm 51\end{array}$ & $\begin{array}{l}377 \\
\pm 96\end{array}$ & 0.050 & & & \\
\hline \multirow{2}{*}{$\begin{array}{c}\text { Treibende } \\
\text { Druckdifferenz } \\
\left(\mathrm{cmH}_{2} \mathrm{O}\right)\end{array}$} & $\begin{array}{r}15,33 \\
\pm 1,03\end{array}$ & $\begin{array}{c}12,61 \\
\pm 1,29\end{array}$ & $\begin{array}{r}12,04 \\
\pm 2,64\end{array}$ & $\begin{array}{c}16,41 \\
\pm 2,95\end{array}$ & $\begin{array}{r}20,01 \\
\pm 7,79\end{array}$ & $\begin{array}{r}24,63 \\
\pm 7,18\end{array}$ & $<0.001$ & \multirow{2}{*}{$<0.001$} & \multirow{2}{*}{$<0.001$} & \multirow{2}{*}{0.334} \\
\hline & $\begin{array}{c}21,52 \\
\pm 5,77\end{array}$ & $\begin{array}{r}16,39 \\
\pm 1,81\end{array}$ & $\begin{array}{r}15,05 \\
\pm 4,02\end{array}$ & $\begin{array}{r}18,00 \\
\pm 2,98\end{array}$ & $\begin{array}{r}21,37 \\
\pm 5,35\end{array}$ & $\begin{array}{r}23,72 \\
\pm 4,05\end{array}$ & 0.007 & & & \\
\hline \multirow{2}{*}{$\begin{array}{l}\text { Treibende trans- } \\
\text { pulmonale } \\
\text { Druckdifferenz } \\
\quad\left(\mathrm{cmH}_{2} \mathrm{O}\right) \\
\end{array}$} & $\begin{array}{c}8,34 \pm \\
1,04\end{array}$ & $\begin{array}{c}6,32 \\
\pm 1,27\end{array}$ & $\begin{array}{c}6,22 \\
\pm 2,19\end{array}$ & $\begin{array}{l}7,54 \\
\pm 2,7\end{array}$ & $\begin{array}{l}8,4 \\
\pm 3\end{array}$ & $\begin{array}{r}12,96 \\
\pm 6,66\end{array}$ & 0.005 & \multirow{2}{*}{$<0.001$} & \multirow{2}{*}{$<0.001$} & \multirow{2}{*}{0.427} \\
\hline & $\begin{array}{r}16,28 \\
\pm 6,33\end{array}$ & $\begin{array}{c}9,40 \\
\pm 2,83\end{array}$ & $\begin{array}{r}9,37 \\
\pm 3,60\end{array}$ & $\begin{array}{c}8,76 \\
\pm 2,84\end{array}$ & $\begin{array}{r}11,23 \\
\pm 2,22\end{array}$ & $\begin{array}{c}15,11 \\
\pm 2,57\end{array}$ & 0.008 & & & \\
\hline \multirow{2}{*}{$\begin{array}{c}\text { Plateaudruck } \\
\text { (Atemwege) } \\
\left(\mathbf{c m H}_{2} \mathbf{O}\right)\end{array}$} & $\begin{array}{c}16,6 \\
\pm 1,41\end{array}$ & $\begin{array}{l}17,78 \\
\pm 1,5\end{array}$ & $\begin{array}{l}20,3 \\
\pm 3 \\
\end{array}$ & $\begin{array}{r}29,34 \\
\pm 3,14\end{array}$ & $\begin{array}{r}35,84 \\
\pm 8,46\end{array}$ & $\begin{array}{r}43,75 \\
\pm 7,42\end{array}$ & $<0.001$ & \multirow[t]{2}{*}{$<0.001$} & \multirow{2}{*}{$<0.001$} & \multirow{2}{*}{0.413} \\
\hline & $\begin{array}{c}22,72 \\
\pm 5,78\end{array}$ & $\begin{array}{l}21,54 \\
\pm 2,12\end{array}$ & $\begin{array}{r}23,38 \\
\pm 4,04 \\
\end{array}$ & $\begin{array}{c}31,04 \\
\pm 3,50\end{array}$ & $\begin{array}{r}37,19 \\
\pm 6,01\end{array}$ & $\begin{array}{r}43,49 \\
\pm 4,54\end{array}$ & $<0.001$ & & & \\
\hline \multirow{2}{*}{$\begin{array}{l}\text { PEEP-bedingtes } \\
\text { Lungenvolumen } \\
\text { (ml) }\end{array}$} & 0 & $\begin{array}{c}80 \\
\pm 27\end{array}$ & $\begin{array}{c}189 \\
\pm 36\end{array}$ & $\begin{array}{c}453 \\
\pm 141\end{array}$ & $\begin{array}{c}651 \\
\pm 200\end{array}$ & $\begin{array}{c}878 \\
\pm 106\end{array}$ & $<0.001$ & \multirow{2}{*}{0.011} & \multirow{2}{*}{$<0.001$} & \multirow{2}{*}{0.478} \\
\hline & 0 & $\begin{array}{c}71 \\
\pm 47 \\
\end{array}$ & $\begin{array}{l}204 \\
\pm 73 \\
\end{array}$ & $\begin{array}{c}512 \\
\pm 106 \\
\end{array}$ & $\begin{array}{c}734 \\
\pm 143 \\
\end{array}$ & $\begin{array}{c}851 \\
\pm 205 \\
\end{array}$ & $<0.001$ & & & \\
\hline \multirow{2}{*}{ EELV (ml) } & $\begin{array}{c}369 \\
\pm 65\end{array}$ & $\begin{array}{r}412 \\
\pm 94 \\
\end{array}$ & $\begin{array}{c}494 \\
\pm 105 \\
\end{array}$ & $\begin{array}{c}828 \\
\pm 147\end{array}$ & $\begin{array}{c}1021 \\
\pm 156\end{array}$ & $\begin{array}{r}1219 \\
\pm 128\end{array}$ & $<0.001$ & \multirow{2}{*}{0.0226} & \multirow{2}{*}{$<0.001$} & \multirow{2}{*}{0.010} \\
\hline & $\begin{array}{r}304 \\
\pm 34 \\
\end{array}$ & $\begin{array}{r}388 \\
\pm 96\end{array}$ & $\begin{array}{c}505 \\
\pm 137\end{array}$ & $\begin{array}{l}910 \\
\pm 98\end{array}$ & $\begin{array}{r}1090 \\
\pm 166\end{array}$ & $\begin{array}{r}1228 \\
\pm 257\end{array}$ & $<0.001$ & & & \\
\hline \multirow{2}{*}{ Stress $\left(\mathbf{c m H}_{2} \mathbf{O}\right)$} & $\begin{array}{c}9,1 \\
\pm 1,3\end{array}$ & $\begin{array}{c}9,7 \\
\pm 2,3\end{array}$ & $\begin{array}{l}12,9 \\
\pm 2,6\end{array}$ & $\begin{array}{c}17,7 \\
\pm 3,9\end{array}$ & $\begin{array}{r}19,5 \\
\pm 4,8\end{array}$ & $\begin{array}{c}28,7 \\
\pm 8,2\end{array}$ & $<0.001$ & \multirow{2}{*}{$\begin{array}{c}< \\
0.001\end{array}$} & \multirow{2}{*}{$<0.001$} & \\
\hline & $\begin{array}{c}17,3 \\
\pm 6,4\end{array}$ & $\begin{array}{r}14,9 \\
\pm 4,3\end{array}$ & $\begin{array}{c}15,1 \\
\pm 6\end{array}$ & $\begin{array}{c}21,3 \\
\pm 4\end{array}$ & $\begin{array}{r}25,6 \\
\pm 3,7\end{array}$ & $\begin{array}{c}33 \\
\pm 5,6\end{array}$ & $<0.001$ & & & $0.00 /$ \\
\hline
\end{tabular}


Tabelle 1 (fortgesetzt)

\begin{tabular}{|c|c|c|c|c|c|c|c|c|c|c|}
\hline Gewählter PEEP & $\mathbf{0}$ & 4 & 7 & 11 & 14 & 18 & \multirow{2}{*}{$\begin{array}{c}\text { P- } \\
\text { Wert }\end{array}$} & \multirow{2}{*}{ Zeit } & \multirow{2}{*}{ PEEP } & \multirow{2}{*}{$\begin{array}{l}\text { Zeit:PEEP- } \\
\text { Interaktion }\end{array}$} \\
\hline Anzahl Schweine & 6 & 6 & 6 & 6 & $6^{*}$ & $6 * *$ & & & & \\
\hline \multirow{2}{*}{ Strain } & $\begin{array}{c}1,0 \\
\pm 0,04\end{array}$ & $\begin{array}{c}1,2 \\
\pm 0,1\end{array}$ & $\begin{array}{c}1,7 \\
\pm 0,2\end{array}$ & $\begin{array}{c}2,2 \\
\pm 0,5\end{array}$ & $\begin{array}{c}2,8 \\
\pm 0,4\end{array}$ & $\begin{array}{c}3,6 \\
\pm 0,4\end{array}$ & $<0.001$ & \multirow{2}{*}{0.379} & \multirow{2}{*}{$<0.001$} & \multirow{2}{*}{$<0.001$} \\
\hline & $\begin{array}{c}1,2 \\
\pm 0,08\end{array}$ & $\begin{array}{c}1,3 \\
\pm 0,27\end{array}$ & $\begin{array}{c}1,7 \\
\pm 0,22\end{array}$ & $\begin{array}{c}2,2 \\
\pm 0,41\end{array}$ & $\begin{array}{c}3,17 \\
\pm 0,72\end{array}$ & $\begin{array}{r}3,23 \\
\pm 0,8 \\
\end{array}$ & $<0.001$ & & & \\
\hline \multirow{2}{*}{$\begin{array}{c}\text { Elastance } \\
\text { (Lunge) } \\
\left(\mathrm{cmH}_{2} \mathrm{O} / \mathrm{l}\right)\end{array}$} & $\begin{array}{l}23,0 \\
\pm 4,8\end{array}$ & $\begin{array}{r}20,9 \\
\pm 3,2\end{array}$ & $\begin{array}{l}25,1 \\
\pm 4,8\end{array}$ & $\begin{array}{l}27,3 \\
\pm 6,1\end{array}$ & $\begin{array}{l}28,3 \\
\pm 6,0\end{array}$ & $\begin{array}{c}49,2 \\
\pm 13,3\end{array}$ & $<0.001$ & \multirow{2}{*}{$<0.001$} & \multirow{2}{*}{0.002} & \multirow{2}{*}{0.499} \\
\hline & $\begin{array}{c}46,0 \\
\pm 23,54 \\
\end{array}$ & $\begin{array}{c}34,6 \\
\pm 7,12 \\
\end{array}$ & $\begin{array}{c}34,1 \\
\pm 0,2 \\
\end{array}$ & $\begin{array}{r}34,6 \\
\pm 9,5 \\
\end{array}$ & $\begin{array}{r}39,6 \\
\pm 8,6 \\
\end{array}$ & $\begin{array}{c}55,6 \\
\pm 13,3\end{array}$ & 0.113 & & & \\
\hline \multirow{2}{*}{$\begin{array}{l}\text { Elastance (Tho- } \\
\operatorname{rax})\left(\mathrm{cmH}_{2} \mathrm{O} / \mathrm{l}\right)\end{array}$} & $\begin{array}{c}18,1 \\
\pm 5,9\end{array}$ & $\begin{array}{r}18,0 \\
\pm 3,8\end{array}$ & $\begin{array}{l}14,5 \\
\pm 2,7\end{array}$ & $\begin{array}{r}18,0 \\
\pm 8,3\end{array}$ & $\begin{array}{r}23,8 \\
\pm 6,5\end{array}$ & $\begin{array}{l}25,7 \\
\pm 4,3\end{array}$ & 0.026 & \multirow{2}{*}{0.005} & \multirow{2}{*}{0.103} & \multirow{2}{*}{0.125} \\
\hline & $\begin{array}{r}13,9 \\
\pm 3,1\end{array}$ & $\begin{array}{r}16,0 \\
\pm 9,8\end{array}$ & $\begin{array}{c}17,1 \\
\pm 3,9\end{array}$ & $\begin{array}{r}15,5 \\
\pm 4,8\end{array}$ & $\begin{array}{r}17,7 \\
\pm 3,9\end{array}$ & $\begin{array}{r}17,3 \\
\pm 3,0\end{array}$ & 0.832 & & & \\
\hline \multirow{2}{*}{$\begin{array}{l}\text { Elastance (Respi- } \\
\text { ratorisches Sys- } \\
\text { tem) }\left(\mathrm{cmH}_{2} \mathrm{O} / \mathbf{l}\right)\end{array}$} & $\begin{array}{r}41,76 \\
\pm 6,69\end{array}$ & $\begin{array}{c}38,95 \\
\pm 2,13\end{array}$ & $\begin{array}{r}39,64 \\
\pm 5,55\end{array}$ & $\begin{array}{r}45,29 \\
\pm 5,44\end{array}$ & $\begin{array}{c}52,11 \\
\pm 11,32\end{array}$ & $\begin{array}{c}74,91 \\
\pm 11,67\end{array}$ & $<0.001$ & \multirow{2}{*}{$<0.001$} & \multirow{2}{*}{$<0.001$} & \multirow{2}{*}{0.445} \\
\hline & $\begin{array}{c}59,94 \\
\pm 23,72 \\
\end{array}$ & $\begin{array}{c}50,63 \\
\pm 5,23\end{array}$ & $\begin{array}{l}51,14 \\
\pm 20,25\end{array}$ & $\begin{array}{r}50,13 \\
\pm 8,04\end{array}$ & $\begin{array}{c}57,25 \\
\pm 11,12\end{array}$ & $\begin{array}{c}72,88 \\
\pm 12,30\end{array}$ & 0.991 & & & \\
\hline \multirow{2}{*}{$\begin{array}{c}\text { Spezifische } \\
\text { Elastance der } \\
\text { Lunge } \\
\left(\mathrm{cmH}_{2} \mathrm{O}\right)\end{array}$} & $\begin{array}{c}8,32 \\
\pm 1,41\end{array}$ & $\begin{array}{c}6,98 \\
\pm 1,94\end{array}$ & $\begin{array}{c}7,58 \\
\pm 2,09\end{array}$ & $\begin{array}{r}10,12 \\
\pm 2,08\end{array}$ & $\begin{array}{c}10,82 \\
\pm 4,46\end{array}$ & $\begin{array}{r}17,05 \\
\pm 5,98\end{array}$ & $<0.001$ & \multirow{2}{*}{$<0.001$} & \multirow{2}{*}{$<0.001$} & \multirow{2}{*}{0.032} \\
\hline & $\begin{array}{r}13,55 \\
\pm 5,67 \\
\end{array}$ & $\begin{array}{r}10,94 \\
\pm 3,15\end{array}$ & $\begin{array}{c}9,70 \\
\pm 4,66\end{array}$ & $\begin{array}{r}13,88 \\
\pm 4,10\end{array}$ & $\begin{array}{c}14,21 \\
\pm 4,34\end{array}$ & $\begin{array}{r}21,46 \\
\pm 8,75\end{array}$ & 0.013 & & & \\
\hline \multirow{2}{*}{$\begin{array}{c}\text { Mechanische } \\
\text { Leistung: Lunge } \\
\text { (J/min })\end{array}$} & $\begin{array}{l}6,21 \\
\pm 1,9 \\
\end{array}$ & $\begin{array}{c}6,94 \\
\pm 2,11 \\
\end{array}$ & $\begin{aligned} & 9,1 \\
& \pm 3,69 \\
&\end{aligned}$ & $\begin{array}{r}15,29 \\
\pm 3,3 \\
\end{array}$ & $\begin{array}{r}17,85 \\
\pm 6,94\end{array}$ & $\begin{array}{r}21,38 \\
\pm 8,5\end{array}$ & $<0.001$ & \multirow{2}{*}{$<0.001$} & \multirow{2}{*}{$<0.001$} & \multirow{2}{*}{0.713} \\
\hline & $\begin{array}{r}11,86 \\
\pm 4,12\end{array}$ & $\begin{array}{c}11,29 \\
\pm 3,88\end{array}$ & $\begin{array}{r}10,88 \\
\pm 4,88\end{array}$ & $\begin{array}{r}17,78 \\
\pm 3,17\end{array}$ & $\begin{array}{c}22,58 \\
\pm 6,32\end{array}$ & $\begin{array}{r}25,56 \\
\pm 6,59\end{array}$ & $<0.001$ & & & \\
\hline \multirow{2}{*}{$\begin{array}{l}\text { Mechanische } \\
\text { Leistung: Respi- } \\
\text { ratorisches Sys- } \\
\text { tem } \\
(\mathrm{J} / \mathrm{min})\end{array}$} & $\begin{array}{c}20,31 \\
\pm 10,57 \\
\end{array}$ & $\begin{array}{l}18,05 \\
\pm 3,99\end{array}$ & $\begin{array}{r}18,39 \\
\pm 7,28\end{array}$ & $\begin{array}{c}31,3 \\
\pm 6,75\end{array}$ & $\begin{array}{c}37,39 \\
\pm 14,22\end{array}$ & $\begin{array}{c}37,56 \\
\pm 11,23\end{array}$ & $<0.001$ & \multirow[b]{2}{*}{$<0.001$} & \multirow[b]{2}{*}{$<0.001$} & \multirow[b]{2}{*}{0.696} \\
\hline & $\begin{array}{r}23,03 \\
\pm 7,33\end{array}$ & $\begin{array}{l}20,29 \\
\pm 2,72\end{array}$ & $\begin{array}{l}21,05 \\
\pm 8,06\end{array}$ & $\begin{array}{r}32,87 \\
\pm 6,88\end{array}$ & $\begin{array}{c}39,52 \\
\pm 13,97\end{array}$ & $\begin{array}{c}39,91 \\
\pm 10,31\end{array}$ & $<0.001$ & & & \\
\hline
\end{tabular}

Für jede Variable werden die Mittelwerte \pm Standardabweichung in den verschiedenen PEEP-Gruppen zu Beginn des Experiments (erste Reihe, hellgrau) und am Ende (zweite Reihe, weiß) angegeben. Die graue Spalte zeigt den P-Wert des einseitigen (one way) ANOVA zu Beginn (erste Reihe) und am Ende des Experiments (zweite Reihe). In den letzten drei Spalten werden die Varianzanalysen mittels linear gemischten Modells für jede Variable einmal nach dem gesetzten PEEP-Wert, nach der Zeit sowie deren Interaktion dargestellt. (Abkürzungen: $\mathrm{V}_{\mathrm{T}}$ : Tidalvolumen; FRC: Funktionelle Residualkapazität; PEEP: Positiver end-exspiratorischer Druck; EILV: End-inspiratorisches Lungenvolumen; EELV: End-exspiratorisches Lungenvolumen; * Zwei Schweine mit einem PEEP von $14 \mathrm{cmH}_{2} \mathrm{O}$ starben nach 30 und $42 \mathrm{~h}$; ** Drei Schweine mit einem PEEP von $18 \mathrm{cmH}_{2} \mathrm{O}$ starben nach $18 \mathrm{~h}$ und eins nach $30 \mathrm{~h}$.) 


\subsection{Die Effekte des PEEP auf den Gasaustausch}

In Abb. 11 stellen sich die untersuchten Parameter des Gasaustausches im zeitlichen Verlauf dar. Darin wird gezeigt, dass das Verhältnis von Sauerstoffpartialdruck und Sauerstoffkonzentration $\left(\mathrm{PaO}_{2} / \mathrm{FiO}_{2}\right.$-Verhältnis) (s. Abb. 11A) in den verschiedenen PEEP-Gruppen unterschiedlich war und im Verlauf leicht, aber signifikant abnahm $(p=0,015)$. Dies war bei der ZEEP-Gruppe besonders auffällig (von $632 \pm 59 \mathrm{mmHg}$ zu $505 \pm 106 \mathrm{~mm} \mathrm{Hg}$; p = 0,028). Der Shunt-Anteil, also das durch die Lungen perfundierte Blut ohne Oxygenierung (s. Abb. 11B), unterschied sich weitestgehend zwischen den PEEP-Gruppen und stieg im Verlauf an.

Die maschinelle Beatmung der Versuchstiere mit den im Studiendesign festgelegten Ventilationseinstellungen führten zu einer Hypokapnie in allen Versuchstieren sowohl zu Beginn als auch im Verlauf des Experiments (s. Abb. 11C). Der aus den kapnometrischen Berechnungen ermittelte Anteil der Totraumventilation am Atemzeitvolumen nahm in allen Versuchsgruppen zu, insbesondere in der PEEP-18-Gruppe ( $<<0,001)$ (s. Abb. 11D). 

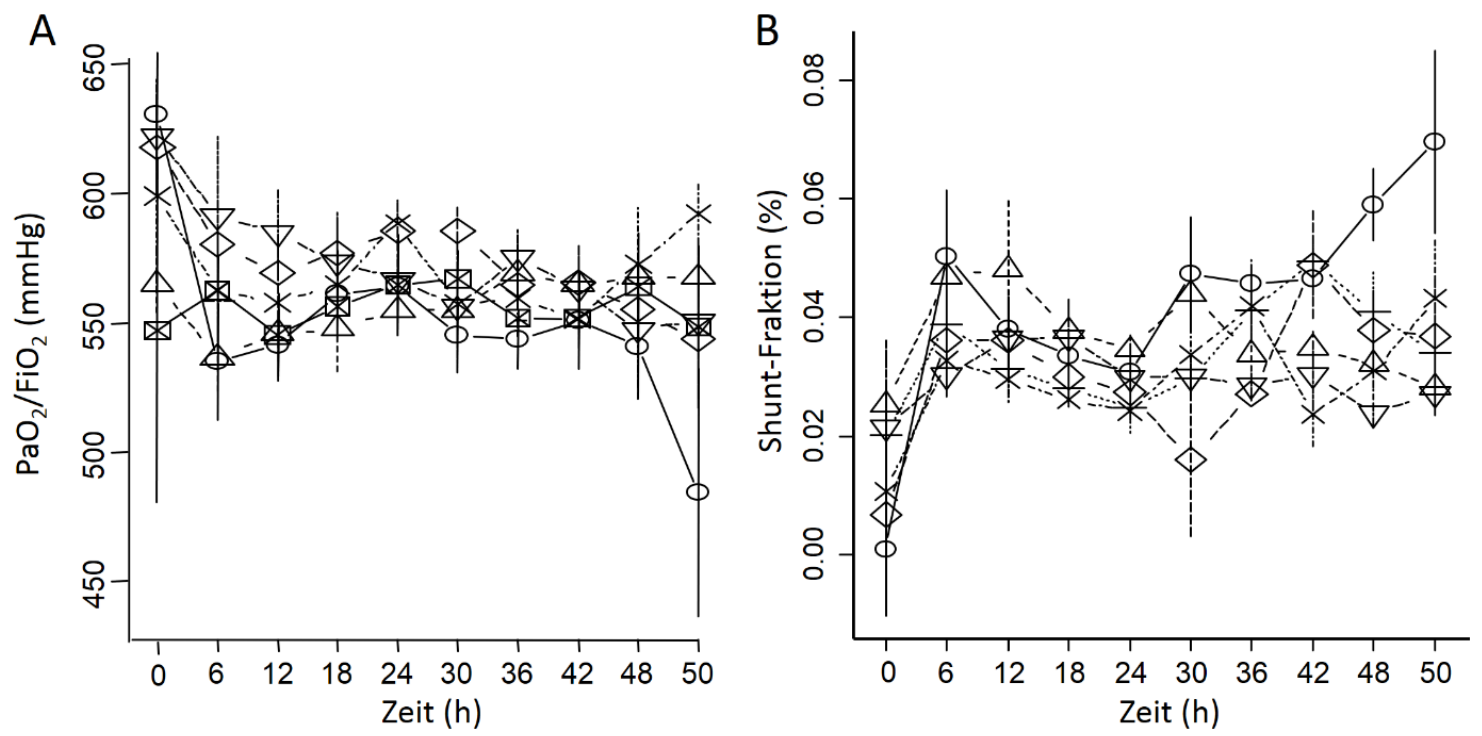

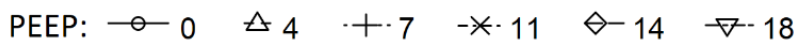
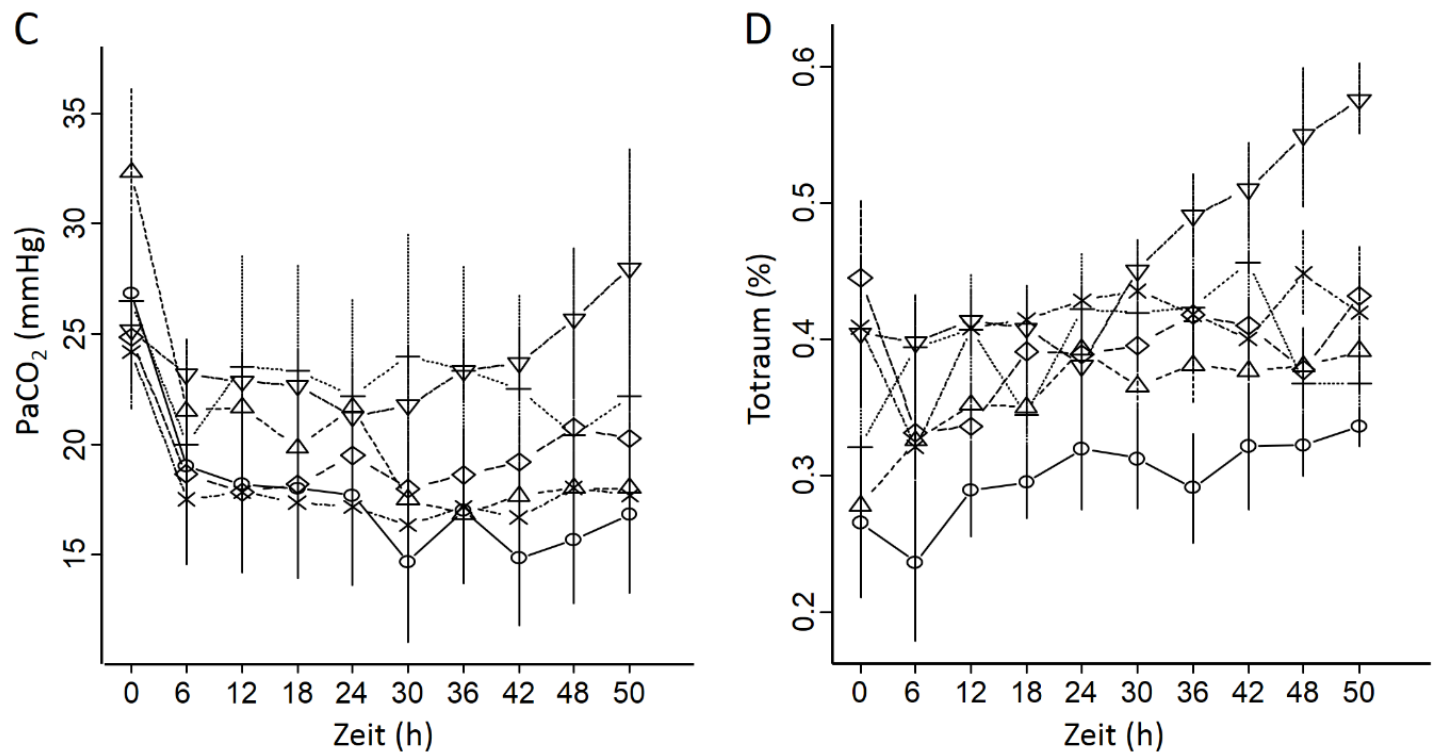

\section{Abbildung 11: Parameter des Gasaustauschs im zeitlichen Verlauf}

In der Abbildung wird das $\mathrm{PaO}_{2} / \mathrm{FiO}_{2}$-Verhältnis (A, Varianzanalyse: Linear gemischtes Modell: Zeit $(p<0,001)$, PEEP $(p=0,216)$, PEEP:Zeit-Interaktion $(p=0,216))$, die Shunt-Fraktion (B, Varianzanalyse: Linear gemischtes Modell: Zeit $(p<0,001)$, PEEP ( $p=0,132)$, PEEP:Zeit-Interaktion $(p=0,016))$, der arterielle Kohlenstoffdioxid-Partialdruck (C, Varianzanalyse: Linear gemischtes Modell: Zeit $(\mathrm{p}<0,001)$, PEEP $(\mathrm{p}=0,553)$, PEEP:Zeit-Interaktion $(\mathrm{p}=0,011))$ und der Totraum (angegeben als Anteil der Totraumventilation am Atemzeitvolumen in Prozent) (D, Varianzanalyse: Linear gemischtes Modell: Zeit ( $p<0,001)$, PEEP $(p<0,001)$, PEEP:Zeit-Interaktion $(p=0,103))$ der verschiedenen PEEP-Gruppen im zeitlichen Verlauf dargestellt. Die Verwendung der Abbildung erfolgt mit freundlicher Genehmigung des Wolters Kluwer Health Verlags (Collino et al. 2019). 
Die Parameter des Säure-Base-Haushalts sind in Abb. 12 dargestellt. Der base excess (BE) (s. Abb. 12A) und der pH-Wert (s. Abb. 10B) waren zu Beginn ähnlich in allen Gruppen, nahmen allerdings im Verlauf stetig ab, obwohl das Laktat annähernd konstant blieb und sich nicht in den PEEP-Gruppen unterschied (s. Abb. 15A).
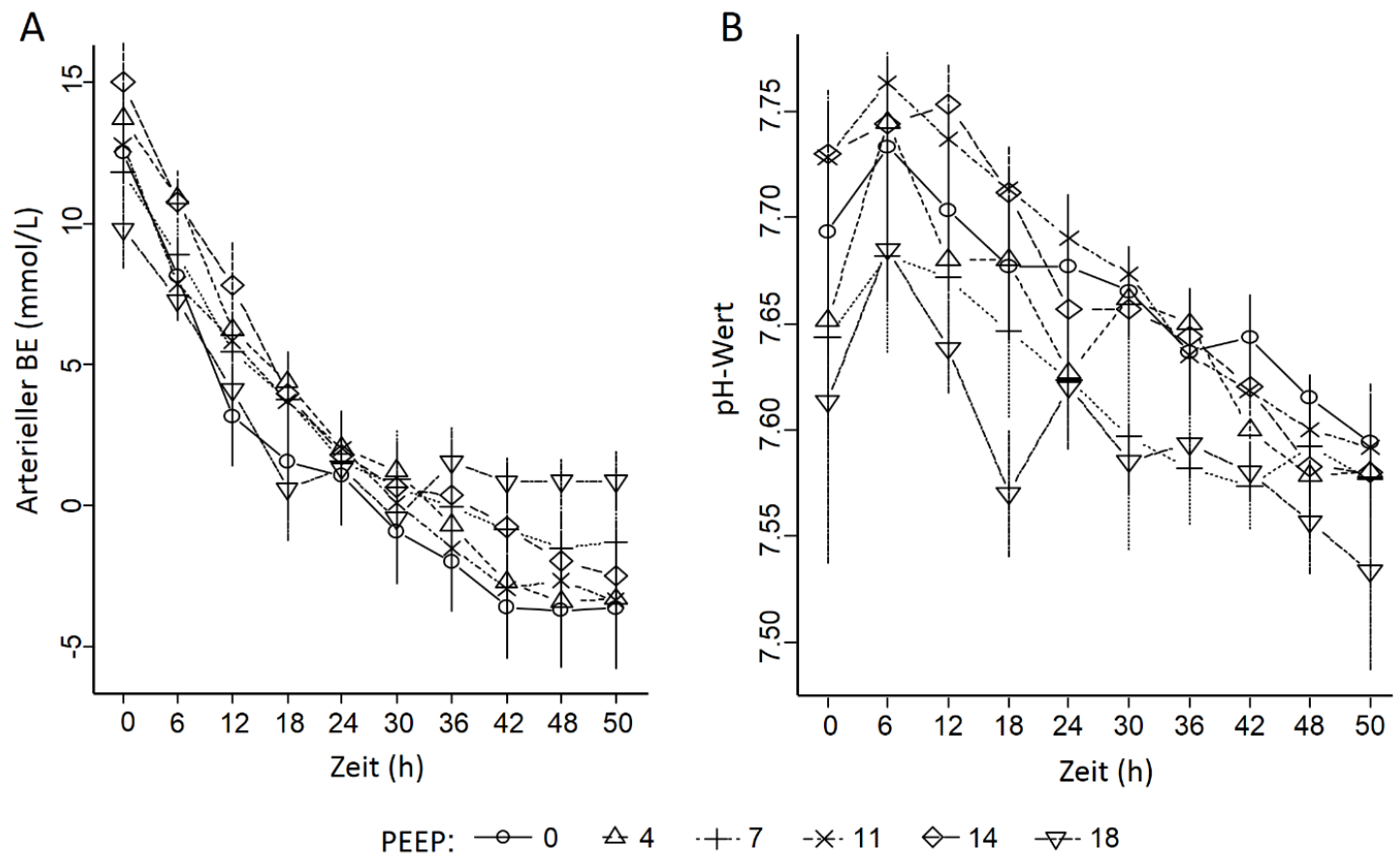

\section{Abbildung 12: Parameter des Säure-Base-Haushalts im zeitlichen Verlauf}

Die obige Grafik zeigt sowohl den arteriellen base excess (BE) (A, Varianzanalyse: Linear gemischtes Modell: Zeit $(\mathrm{p}<0,001)$, PEEP $(\mathrm{p}=0,899)$, PEEP:Zeit-Interaktion $(\mathrm{p}=0,006))$ als auch den $\mathrm{pH}-$ Wert (B, Varianzanalyse: Linear gemischtes Modell: Zeit $(p<0,001)$, PEEP ( $p=0,062)$, PEEP:ZeitInteraktion $(\mathrm{p}=0,755))$ in den verschiedenen PEEP-Gruppen im zeitlichen Verlauf. Die Verwendung der Abbildung erfolgt mit freundlicher Genehmigung des Wolters Kluwer Health Verlags (Collino et al. 2019).

In der Tabelle 2 werden die Auswirkungen des PEEP auf den Gasaustausch dargestellt. Die Parameter im Zusammenhang mit der Oxygenierung waren, obwohl sie sich in den Gruppen sehr unterschiedlich waren, dennoch in den Normbereichen. Allerdings waren alle Schweine hypokapnisch (s. Abb. 11 und s. Tabelle 2). 
Tabelle 2: Parameter des Gasaustauschs

\begin{tabular}{|c|c|c|c|c|c|c|c|c|c|c|}
\hline $\begin{array}{c}\text { PEEP } \\
\left(\mathrm{cmH}_{2} \mathrm{O}\right)\end{array}$ & 0 & 4 & 7 & 11 & 14 & 18 & $\begin{array}{c}\text { P- } \\
\text { Wert }\end{array}$ & Zeit & PEEP & $\begin{array}{c}\text { Zeit: } \\
\text { PEEP } \\
\text { Interaktion }\end{array}$ \\
\hline \multirow{2}{*}{$\begin{array}{c}\mathrm{PaO}_{2} / \mathrm{FiO}_{2} \\
(\mathrm{mmHg})\end{array}$} & $\begin{array}{l}632 \\
\pm 59\end{array}$ & $\begin{array}{l}567 \\
\pm 36\end{array}$ & $\begin{array}{l}601 \\
\pm 87\end{array}$ & $\begin{array}{l}620 \\
\pm 55\end{array}$ & $\begin{array}{l}624 \\
\pm 61\end{array}$ & $\begin{array}{c}549 \\
\pm 163\end{array}$ & 0,49 & \multirow{2}{*}{$<0.001$} & \multirow{2}{*}{0.216} & \multirow{2}{*}{0.216} \\
\hline & $\begin{array}{c}505 \\
\pm 106\end{array}$ & $\begin{array}{l}569 \\
\pm 32\end{array}$ & $\begin{array}{l}594 \\
\pm 30\end{array}$ & $\begin{array}{l}545 \\
\pm 60\end{array}$ & $\begin{array}{l}565 \\
\pm 52\end{array}$ & $\begin{array}{l}565 \\
\pm 43\end{array}$ & 0,26 & & & \\
\hline \multirow{2}{*}{$\begin{array}{c}\text { Shunt Fraktion } \\
(\%)\end{array}$} & $\begin{array}{c}0 \\
\pm 0\end{array}$ & $\begin{array}{l}2,5 \\
\pm 2,5\end{array}$ & $\begin{array}{c}2,0 \\
\pm 2,4\end{array}$ & $\begin{array}{c}1,1 \\
\pm 2,0\end{array}$ & $\begin{array}{c}0,7 \\
\pm 2,4\end{array}$ & $\begin{array}{c}2,1 \\
\pm 3,7\end{array}$ & 0,57 & \multirow{2}{*}{$<0.001$} & \multirow{2}{*}{0.132} & \multirow{2}{*}{0.016} \\
\hline & $\begin{array}{c}6,8 \\
\pm 3,1\end{array}$ & $\begin{array}{c}2,8 \\
\pm 1,1\end{array}$ & $\begin{array}{l}3,4 \\
\pm 1\end{array}$ & $\begin{array}{l}4,3 \\
\pm 2,5\end{array}$ & $\begin{array}{r}4,3 \\
\pm 2,8\end{array}$ & $\begin{array}{c}3,4 \\
\pm 1,7\end{array}$ & 0,05 & & & \\
\hline \multirow{2}{*}{$\begin{array}{c}\text { Venöse } O_{2} \text {-Sätti- } \\
\text { gung }(\%)\end{array}$} & $\begin{array}{l}92,8 \\
\pm 4,6\end{array}$ & $\begin{array}{l}86,7 \\
\pm 3,9\end{array}$ & $\begin{array}{l}89,7 \\
\pm 5,9\end{array}$ & $\begin{array}{l}92,5 \\
\pm 7,1\end{array}$ & $\begin{array}{l}94,6 \\
\pm 1,1\end{array}$ & $\begin{array}{c}86,7 \\
\pm 10,1\end{array}$ & 0,19 & \multirow{2}{*}{$<0.001$} & \multirow{2}{*}{0.500} & \multirow{2}{*}{0.504} \\
\hline & $\begin{array}{l}84,3 \\
\pm 8,8\end{array}$ & $\begin{array}{r}80 \\
\pm 13,2\end{array}$ & $\begin{array}{l}88,8 \\
\pm 5,9\end{array}$ & $\begin{array}{l}85,0 \\
\pm 7,3\end{array}$ & $\begin{array}{l}82,7 \\
\pm 6,5\end{array}$ & $\begin{array}{r}82,7 \\
\pm 12,9\end{array}$ & 0,71 & & & \\
\hline \multirow{2}{*}{$\begin{array}{l}\text { Atemzeitvolu- } \\
\text { men (L/min) }\end{array}$} & $\begin{array}{l}11,2 \\
\pm 1,8\end{array}$ & $\begin{array}{c}9,7 \\
\pm 1,3\end{array}$ & $\begin{array}{c}9,3 \\
\pm 2,7\end{array}$ & $\begin{array}{l}10,8 \\
\pm 1,1\end{array}$ & $\begin{array}{l}11,2 \\
\pm 2,1\end{array}$ & $\begin{array}{c}9,8 \\
\pm 1,8\end{array}$ & 0,33 & \multirow{2}{*}{0.656} & \multirow{2}{*}{0.330} & \multirow{2}{*}{0.346} \\
\hline & $\begin{array}{l}11,2 \\
\pm 1,7\end{array}$ & $\begin{array}{c}9,8 \\
\pm 1,2\end{array}$ & $\begin{array}{c}9,4 \\
\pm 2,7\end{array}$ & $\begin{array}{l}10,8 \\
\pm 1,1\end{array}$ & $\begin{array}{l}11,2 \\
\pm 2,2\end{array}$ & $\begin{array}{c}9,9 \\
\pm 1,7\end{array}$ & 0,37 & & & \\
\hline
\end{tabular}


Tabelle 2 (fortgesetzt)

\begin{tabular}{|c|c|c|c|c|c|c|c|c|c|c|}
\hline $\begin{array}{c}\text { PEEP } \\
\left(\mathrm{cmH}_{2} \mathrm{O}\right)\end{array}$ & 0 & 4 & 7 & 11 & 14 & 18 & $\begin{array}{c}\text { P- } \\
\text { Wert }\end{array}$ & Zeit & PEEP & $\begin{array}{c}\text { Zeit: } \\
\text { PEEP } \\
\text { Interaktion }\end{array}$ \\
\hline \multirow{2}{*}{$\begin{array}{c}\text { Anteil des } \\
\text { Totraums (\%) }\end{array}$} & $\begin{array}{c}0,27 \\
\pm 0,13\end{array}$ & $\begin{array}{c}0,28 \\
\pm 0,09\end{array}$ & $\begin{array}{c}0,32 \\
\pm 0,20\end{array}$ & $\begin{array}{c}0,41 \\
\pm 0,08\end{array}$ & $\begin{array}{c}0,44 \\
\pm 0,08\end{array}$ & $\begin{array}{c}0,40 \\
\pm 0,14\end{array}$ & 0,09 & \multirow{2}{*}{$<0.001$} & \multirow{2}{*}{0.019} & \multirow{2}{*}{0.103} \\
\hline & $\begin{array}{l}0,35 \\
\pm 0,03\end{array}$ & $\begin{array}{c}0,41 \\
\pm 0,06\end{array}$ & $\begin{array}{c}0,36 \\
\pm 0,06\end{array}$ & $\begin{array}{c}0,43 \\
\pm 0,11\end{array}$ & $\begin{array}{c}0,45 \\
\pm 0,03\end{array}$ & $\begin{array}{c}0,55 \\
\pm 0,08\end{array}$ & $<0,001$ & & & \\
\hline \multirow{2}{*}{$\mathrm{PaCO}_{2}(\mathrm{mmHg})$} & $\begin{array}{l}27 \\
\pm 9\end{array}$ & $\begin{array}{c}32 \\
\pm 10\end{array}$ & $\begin{array}{l}27 \\
\pm 8\end{array}$ & $\begin{array}{l}24 \\
\pm 7\end{array}$ & $\begin{array}{l}25 \\
\pm 7\end{array}$ & $\begin{array}{l}25 \\
\pm 3\end{array}$ & 0,46 & \multirow{2}{*}{$<0.001$} & \multirow{2}{*}{0.533} & \multirow{2}{*}{0.011} \\
\hline & $\begin{array}{l}16 \\
\pm 7\end{array}$ & $\begin{array}{l}18 \\
\pm 3\end{array}$ & $\begin{array}{c}22 \\
\pm 11\end{array}$ & $\begin{array}{l}18 \\
\pm 2\end{array}$ & $\begin{array}{l}19 \\
\pm 6\end{array}$ & $\begin{array}{l}25 \\
\pm 8\end{array}$ & 0,24 & & & \\
\hline \multirow{2}{*}{$\begin{array}{c}\text { Arterieller pH- } \\
\text { Wert }\end{array}$} & $\begin{array}{l}7,69 \\
\pm 0,08\end{array}$ & $\begin{array}{c}7,65 \\
\pm 0,10\end{array}$ & $\begin{array}{c}7,64 \\
\pm 0,12\end{array}$ & $\begin{array}{c}7,73 \\
\pm 0,08\end{array}$ & $\begin{array}{r}7,73 \\
\pm 0,07\end{array}$ & $\begin{array}{c}7,61 \\
\pm 0,19\end{array}$ & 0,38 & \multirow{2}{*}{$<0.001$} & \multirow{2}{*}{0.062} & \multirow{2}{*}{0.755} \\
\hline & $\begin{array}{l}7,60 \\
\pm 0,05\end{array}$ & $\begin{array}{c}7,58 \\
\pm 0,03\end{array}$ & $\begin{array}{c}7,58 \\
\pm 0,08\end{array}$ & $\begin{array}{c}7,59 \\
\pm 0,01\end{array}$ & $\begin{array}{c}7,60 \\
\pm 0,07\end{array}$ & $\begin{array}{c}7,54 \\
\pm 0,08\end{array}$ & 0,49 & & & \\
\hline \multirow{2}{*}{$\begin{array}{c}\text { Arterieller BE } \\
\qquad(\mathrm{mmol} / \mathrm{L})\end{array}$} & $\begin{array}{l}12,5 \\
\pm 1,9\end{array}$ & $\begin{array}{l}13,7 \\
\pm 2,9\end{array}$ & $\begin{array}{l}11,8 \\
\pm 1,8\end{array}$ & $\begin{array}{l}12,8 \\
\pm 2,6\end{array}$ & $\begin{array}{l}15,0 \\
\pm 3,4\end{array}$ & $\begin{array}{c}9,8 \\
\pm 3,7\end{array}$ & 0,11 & \multirow{2}{*}{$<0.001$} & \multirow{2}{*}{0.899} & \multirow{2}{*}{0.006} \\
\hline & $\begin{array}{l}-3,7 \\
\pm 4,2\end{array}$ & $\begin{array}{l}-3,3 \\
\pm 2,8\end{array}$ & $\begin{array}{l}-1,3 \\
\pm 5,8\end{array}$ & $\begin{array}{l}-3,4 \\
\pm 1,9\end{array}$ & $\begin{array}{l}-2,3 \\
\pm 3,4\end{array}$ & $\begin{array}{l}-0,7 \\
\pm 4,0\end{array}$ & 0,69 & & & \\
\hline
\end{tabular}

Für jede Variable werden die Mittelwerte \pm Standardabweichung in den verschiedenen PEEP-Gruppen zu Beginn des Experiments (erste Reihe, hellgrau) und am Ende (zweite Reihe, weiß) angegeben. Die graue Spalte zeigt den P-Wert des einseitigen (one way) ANOVA zu Beginn (erste Reihe) und am Ende des Experiments (zweite Reihe). In den letzten drei Spalten werden die Varianzanalysen mittels linear gemischten Modells für jede Variable einmal nach dem gesetzten PEEP-Wert, nach der Zeit sowie deren Interaktion dargestellt. (Abkürzungen: $\mathrm{PaO}_{2}$ : Arterieller Sauerstoffpartialdruck; $\mathrm{SatO}_{2}$ : Sauerstoff-Hämoglobin-Sättigung; $\mathrm{PaCO}_{2}$ : Arterieller Kohlenstoffdioxidpartialdruck; BE: Base excess). 


\subsection{Hämodynamische Parameter}

In Abb. 13 werden die verschiedenen untersuchten hämodynamischen Parameter des Körperkreislaufes im zeitlichen Verlauf präsentiert. Dabei zeigt sich, dass das Herz-Zeit-Volumen (s. Abb. 13A) in den verschiedenen Gruppen zu Beginn ähnliche Werte annahm und im Verlauf signifikant abnahm ( $\mathrm{p}<0,001)$. Der mittlere arterielle Druck (s. Abb. 13B) war zu Beginn unterschiedlich in den verschiedenen Gruppen $(\mathrm{p}<0,001)$. Zwar nahm der Druck im Verlauf signifikant ab, aber es ließ sich kein PEEP-assoziiertes Muster nachweisen.

Um diese arteriellen Blutdruckwerte aufrechtzuerhalten, mussten sowohl der Volumenersatz als auch der Einsatz von Noradrenalin mit zunehmenden gewählten PEEP signifikant erhöht werden (s. Abb. 13C und 13D). Allerdings waren in der Gruppe mit einem PEEP von 0 und $4 \mathrm{Cm} \mathrm{H}_{2} \mathrm{O}$ sehr wenig Noradrenalin notwendig.

In der Tabellen 3 wird eine Übersicht der hämodynamischen Parameter in den verschiedenen PEEP-Gruppen zu Beginn und am Ende des Experimentes dargestellt. 

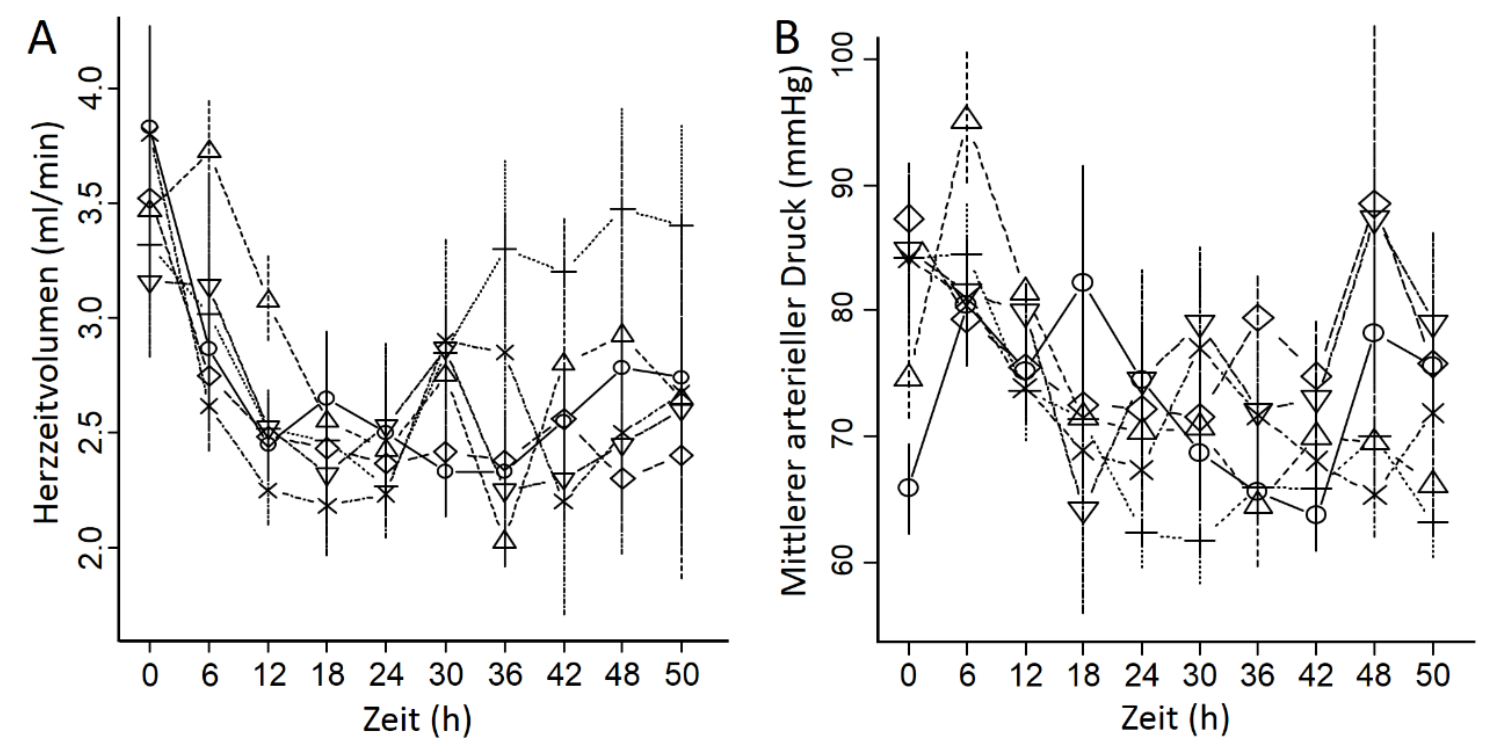

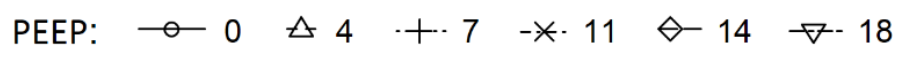
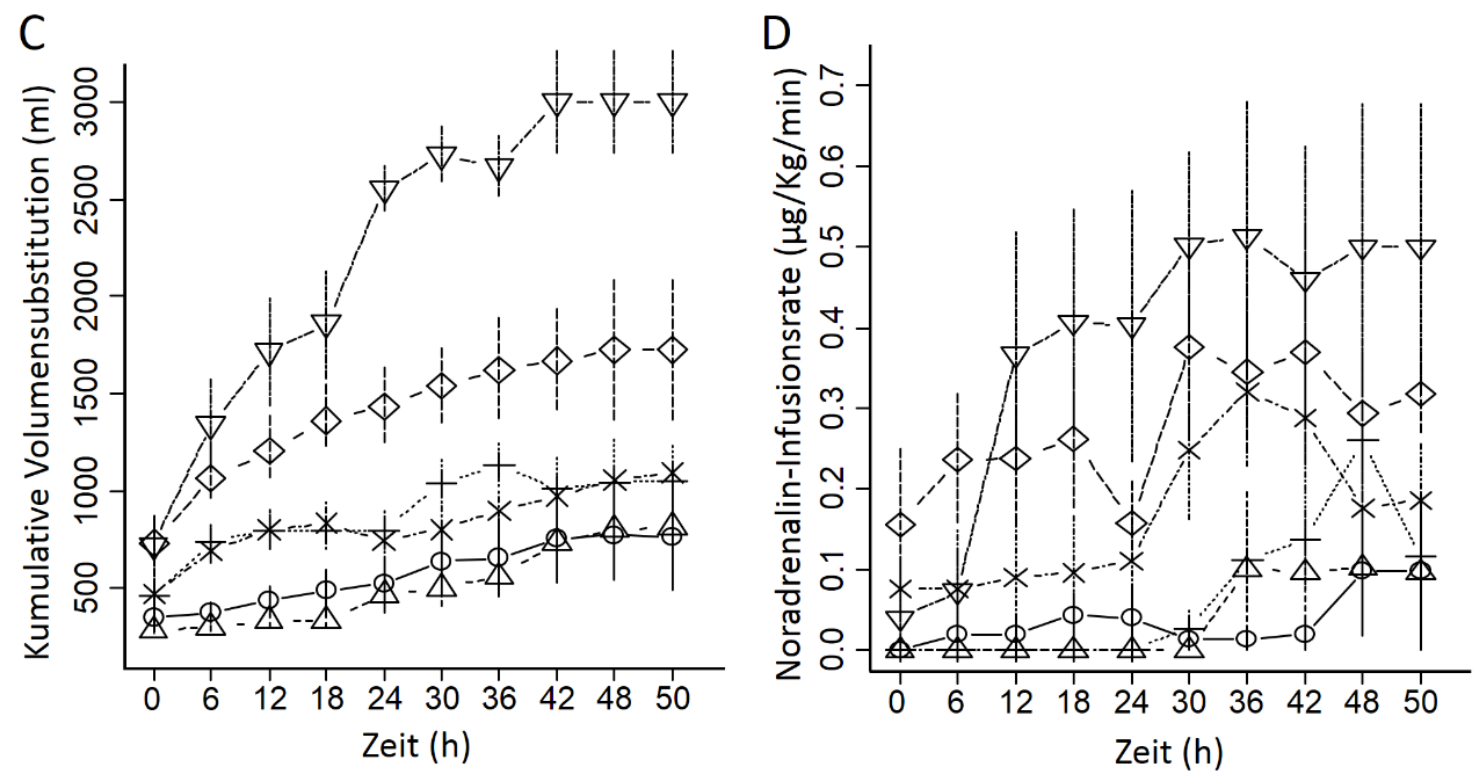

Abbildung 13: Hämodynamische Parameter des Körperkreislaufes im zeitlichen Verlauf

In der Abbildung werden das Herz-Zeit-Volumen (A, Varianzanalyse: Linear gemischtes Modell: Zeit $(\mathrm{p}<0,001), \operatorname{PEEP}(\mathrm{p}=0,684)$, PEEP:Zeit-Interaktion $(\mathrm{p}=0,315))$, der mittlere arterielle Druck (B, Varianzanalyse: Linear gemischtes Modell: Zeit ( $p<0,001)$, PEEP ( $p=0,543)$, PEEP:Zeit-Interaktion ( $\mathrm{p}=0,011)$ ), der kumulative Volumenersatz (C, Varianzanalyse: Linear gemischtes Modell: Zeit $(\mathrm{p}<0,001)$, PEEP $(\mathrm{p}<0,001)$, PEEP:Zeit-Interaktion $(\mathrm{p}<0,001))$ sowie der Einsatz von Noradrenalin (D, Varianzanalyse: Linear gemischtes Modell: Zeit ( $p<0,001)$, PEEP ( $p<0,001)$, PEEP:ZeitInteraktion $(p=0,003))$ in den verschiedenen PEEP-Gruppen im zeitlichen Verlauf dargestellt. Die Verwendung der Abbildung erfolgt mit freundlicher Genehmigung des Wolters Kluwer Health Verlags (Collino et al. 2019). 
Im Lungenkreislauf konnten wir in Bezug auf die hämodynamische Situation, die in Abbildung 14 dargestellten Beobachten registrieren. Sowohl der mittlere pulmonale Druck (s. Abb. 14A) als auch der Wedge-Druck (s. Abb. 14B) waren erhöht, unterschieden sich in den verschiedenen PEEP-Gruppen und stiegen mit zunehmender Zeit in der Tendenz weiter an.

A

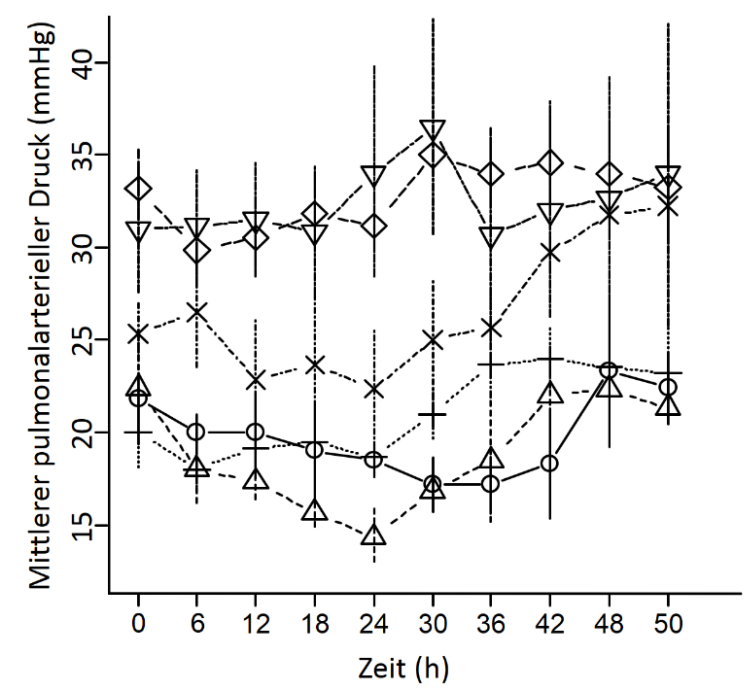

B

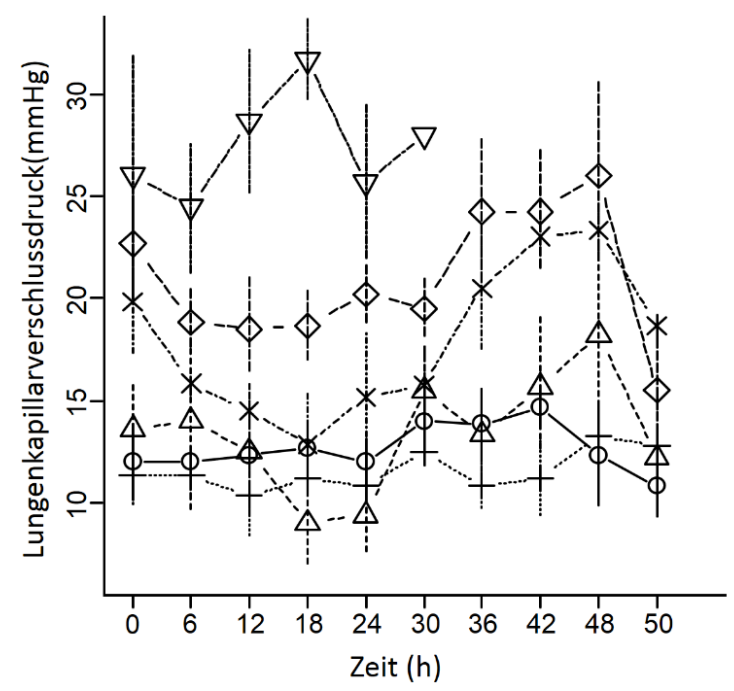

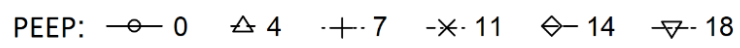

\section{Abbildung 14: Gefäßdrücke im pulmonalen Kreislauf im zeitlichen Verlauf}

Hier wird der mittlere pulmonale Druck (A, Varianzanalyse: Linear gemischtes Modell: Zeit ( $\mathrm{p}<$ 0,001), PEEP ( $\mathrm{p}<0,001)$, PEEP:Zeit-Interaktion $(\mathrm{p}=0,452))$ und der Wedge-Druck (B, Varianzanalyse: Linear gemischtes Modell: Zeit $(\mathrm{p}=0,0251)$, PEEP $(\mathrm{p}<0,001)$, PEEP:Zeit-Interaktion ( $\mathrm{p}=$ $0,961)$ ) in den verschiedenen PEEP-Gruppen über die Dauer des Experiments gezeigt. Die dargestellten Daten zeigen den Mittelwert und Standardfehler. Die Verwendung der Abbildung erfolgt mit freundlicher Genehmigung des Wolters Kluwer Health Verlags (Collino et al. 2019). 
Abb. 15 zeigt zum einen das arterielle Laktat-Level (s. Abb. 15A) und die Flüssigkeitsbilanzierung (s. Abb. 15B). Es wird deutlich, dass das arterielle Laktat bis auf einen vermeintlichen Ausreißer relativ konstant über die Dauer es Experimentes blieb.

A

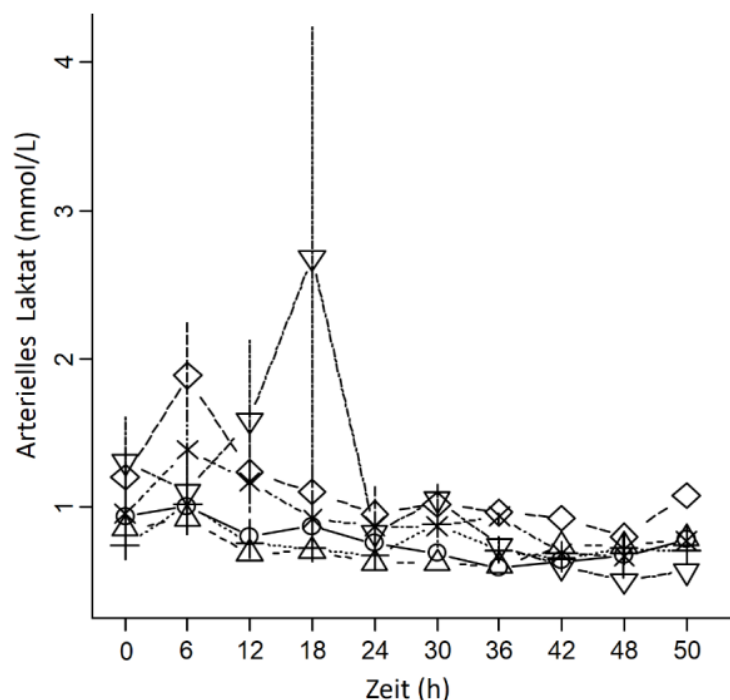

B

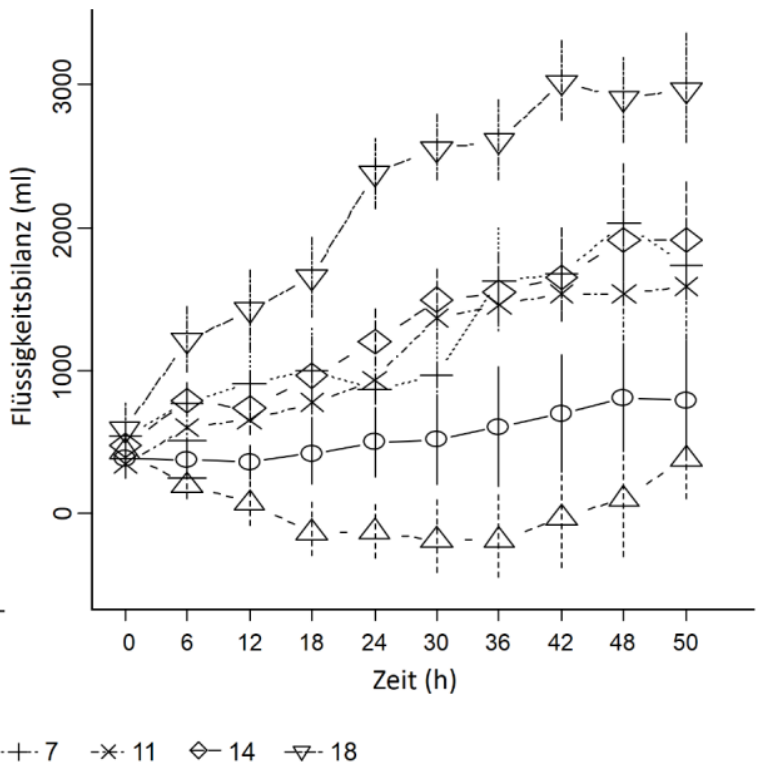

\section{Abbildung 15: Arterielles Laktat und Flüssigkeitsbilanz}

Die obigen Grafiken zeigen zum einen das arterielle Laktat (A, Varianzanalyse: Linear gemischtes Modell: Zeit $(p=0,002)$, PEEP $(p=0,362)$, PEEP:Zeit-Interaktion $(p=0,037))$ und zum anderen die Flüssigkeitsbilanz (B, Varianzanalyse: Linear gemischtes Modell: Zeit $(\mathrm{p}<0,001)$, PEEP ( $\mathrm{p}<$ 0,001), PEEP:Zeit-Interaktion ( $\mathrm{p}<0,001)$ ) der verschiedenen PEEP-Gruppen im zeitlichen Verlauf. Die dargestellten Daten zeigen den Mittelwert und Standardfehler. Die Verwendung der Abbildung erfolgt mit freundlicher Genehmigung des Wolters Kluwer Health Verlags (Collino et al. 2019). 
Tabelle 3: Hämodynamische Parameter

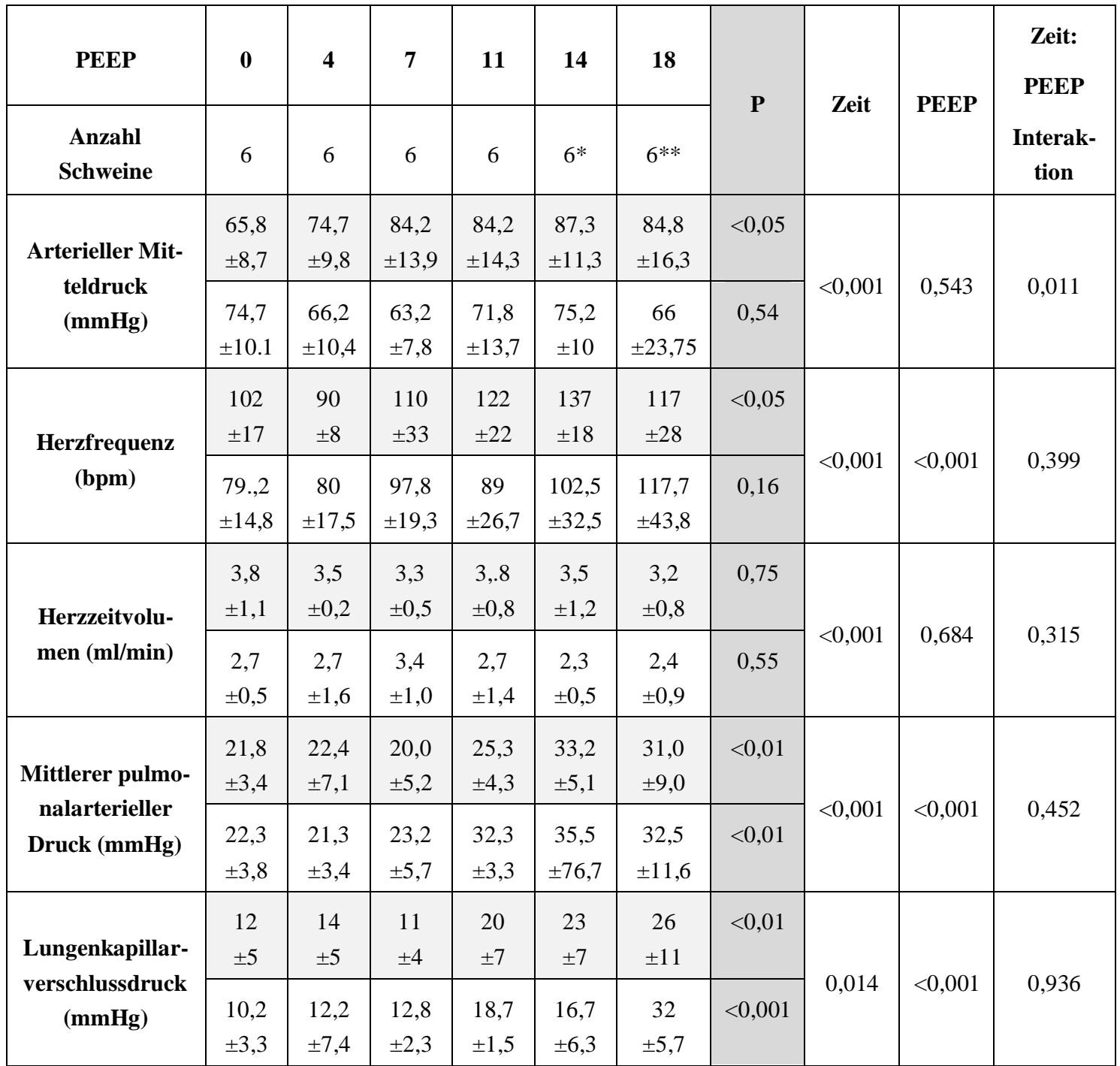


Tabelle 3 (fortgesetzt)

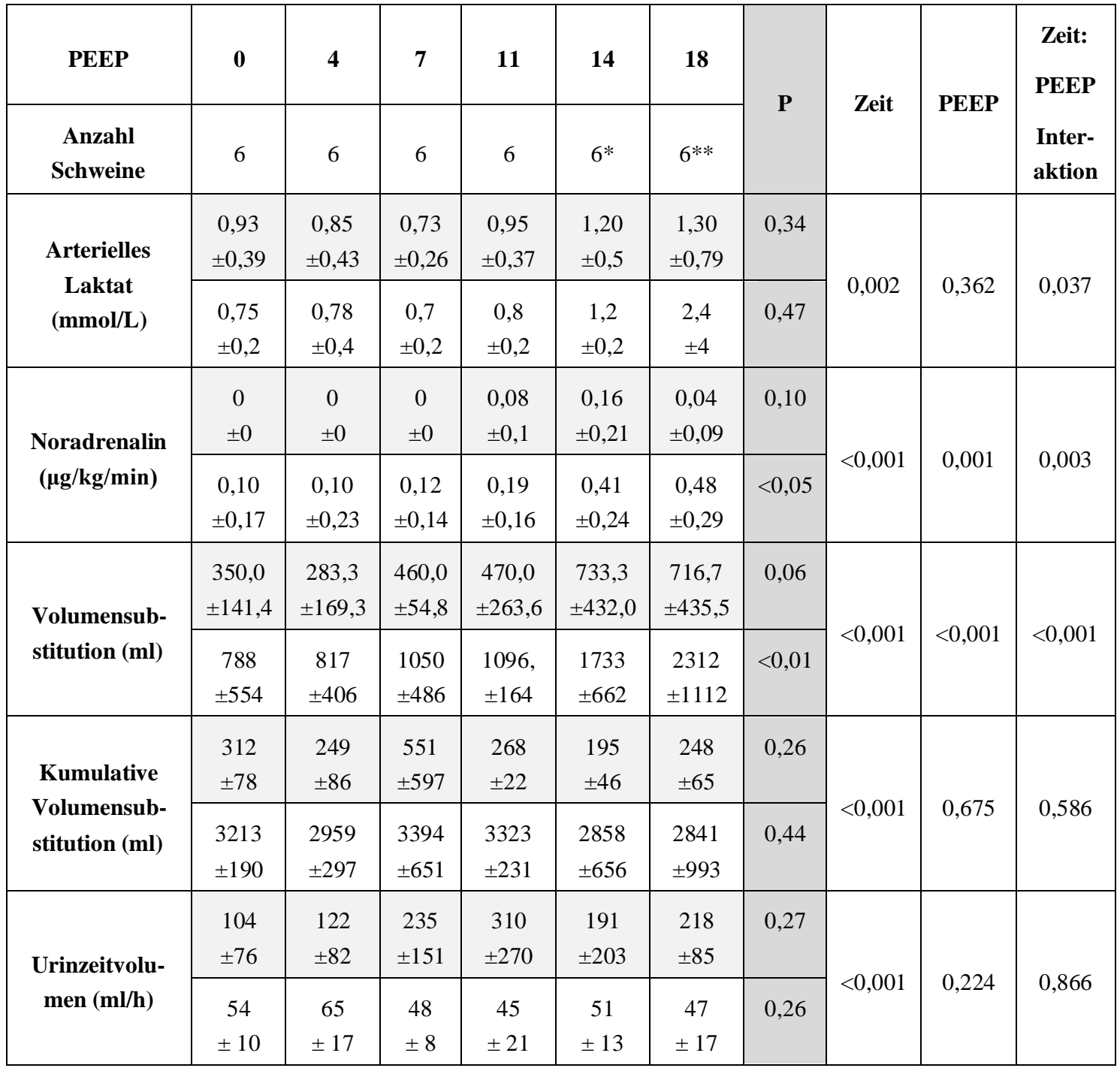

Für jede Variable werden die Mittelwerte \pm Standardabweichung in den verschiedenen PEEP-Gruppen zu Beginn des Experiments (erste Reihe, hellgrau) und am Ende (zweite Reihe, weiß) angegeben. Die graue Spalte zeigt den P-Wert des einseitigen (one way) ANOVA zu Beginn (erste Reihe) und am Ende des Experiments (zweite Reihe). In den letzten drei Spalten werden die Varianzanalysen mittels linear gemischten Modells für jede Variable einmal nach dem gesetzten PEEP-Wert, nach der Zeit sowie deren Interaktion dargestellt. Es wurden keine PEEP-Zeit-Interaktionen beobachtet. * Zwei Schweine mit einem PEEP von $14 \mathrm{cmH}_{2} \mathrm{O}$ starben nach 30 und $42 \mathrm{~h}$; ** Drei Schweine mit einem PEEP von $18 \mathrm{cmH}_{2} \mathrm{O}$ starben nach $18 \mathrm{~h}$ und eins nach $30 \mathrm{~h}$. 


\subsection{PEEP und seine Auswirkungen auf die Lungenstruktur}

\subsubsection{Makroskopische Untersuchungen}

Einen repräsentativen Überblick über den makroskopischen Aspekt der Lungen der verschiedenen PEEP-Gruppen bietet Abbildung 16.

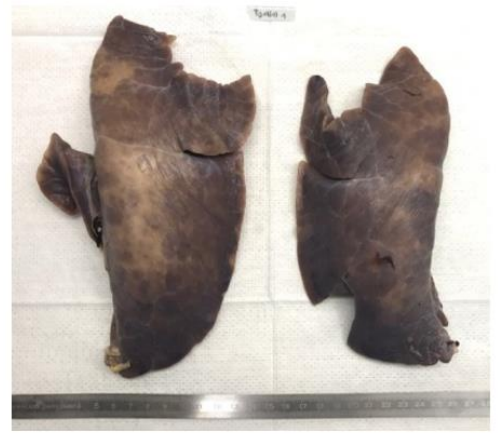

ZEEP (Schwein 14)

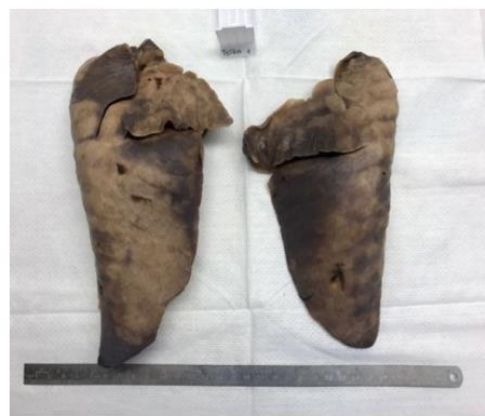

$11 \mathrm{cmH}_{2} \mathrm{O}$ (Schwein 22)

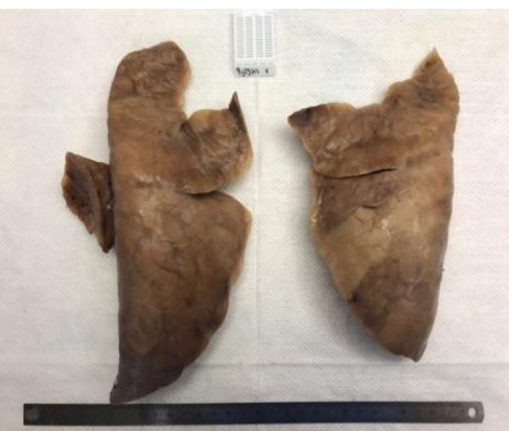

$4 \mathrm{cmH}_{2} \mathrm{O}$ (Schwein 29)

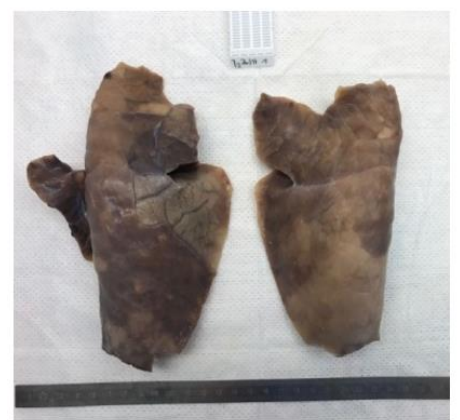

$14 \mathrm{cmH}_{2} \mathrm{O}$ (Schwein 36)

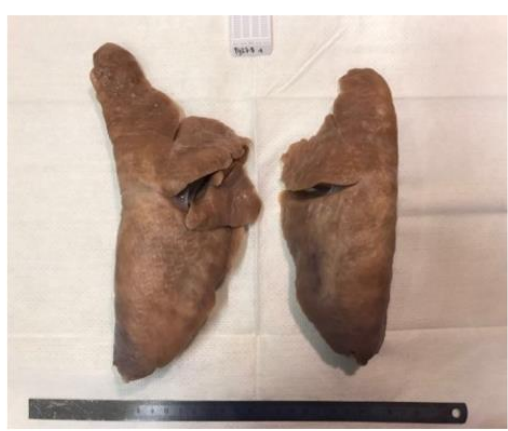

$7 \mathrm{cmH}_{2} \mathrm{O}$ (Schwein 27)

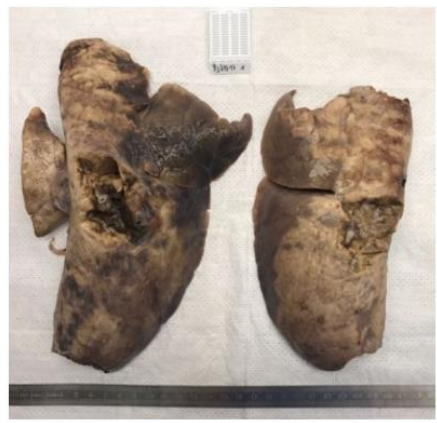

$18 \mathrm{cmH}_{2} \mathrm{O}$ (Schwein 34)

Abbildung 16: Makroskopischer Überblick

In den obigen Fotografien wurde für die unterschiedlichen PEEP-Gruppen jeweils ein repräsentatives Lungen-Paar ausgewählt und mit der jeweiligen Schweine-Nummer gekennzeichnet. Die Verwendung der Abbildung erfolgt mit freundlicher Genehmigung des Wolters Kluwer Health Verlags (Collino et al. 2019). 


\subsubsection{Mikroskopische Untersuchungen}

Die histopathologische Untersuchung in Bezug auf die untersuchten Läsionsarten ergab keinen Unterschied, weder zwischen linker und rechter Lunge noch in verschiedenen Lungenregionen (apikale, mittlere und basale Abschnitte). Die aufgetretenen Lungenschäden waren einheitlich zwischen abhängigen und nichtabhängigen Lungenarealen verteilt. Aus diesem Grund wurden die histologischen Ergebnisse zusammen betrachtet, ungeachtet ihrer anatomischen Position. Einen Überblick über die mikroskopisch beobachteten lungenstrukturellen Veränderungen zeigt die Abbildung 17. Am häufigsten konnten Gefäßstauungen (s. Abb. 18A), gefolgt von inflammatorischen Zellinfiltrationen (s. Abb. 18B), alveolären Kollapsen/Atelektasenbildungen (s. Abb. 18C) sowie septalen Dilatationen/Rupturen (s. Abb. 18D) nachgewiesen werden. Ein wiederkehrendes Verteilungsmuster in Bezug auf die unterschiedlichen PEEP-Gruppen zeigte sich bei diesen Läsionen. Ein Großteil der aufgetretenen Läsionen trat bei der ZEEP-Gruppe auf (s. Abb. 18). Ein minimales Vorkommen an strukturellen Schäden wurde bei den Versuchstieren mit einem PEEP von $4 \mathrm{~cm} \mathrm{H}_{2} \mathrm{O}$ beobachtet, wohingegen das Auftreten der Veränderungen bei höheren PEEP-Werten wieder wahrscheinlicher wurde. Die Häufigkeitsverringerung beim direkten Vergleich von ZEEP zu PEEP $4 \mathrm{cmH}_{2} 0$ war signifikant für Gefäßstauung ( $\left.\mathrm{p}<0,001\right)$, inflammatorische Reaktionen $(\mathrm{p}<0,001)$, Atelektasenbildung $(\mathrm{p}<0,001)$, septale Rupturen $(\mathrm{p}=0,041)$, emphysenartige Veränderungen $(p<0,001)$ sowie intravaskuläre Thromben $(p<0,001)$. Das Auftreten von Gefäßstauungen, Entzündungen, Atelektasen sowie Rupturen nahm beim weiteren Erhöhen des PEEP von $4 \mathrm{zu} 14-18 \mathrm{~cm} \mathrm{H}_{2} 0$ signifikant $\mathrm{zu}(\mathrm{p}<0,001)$. 


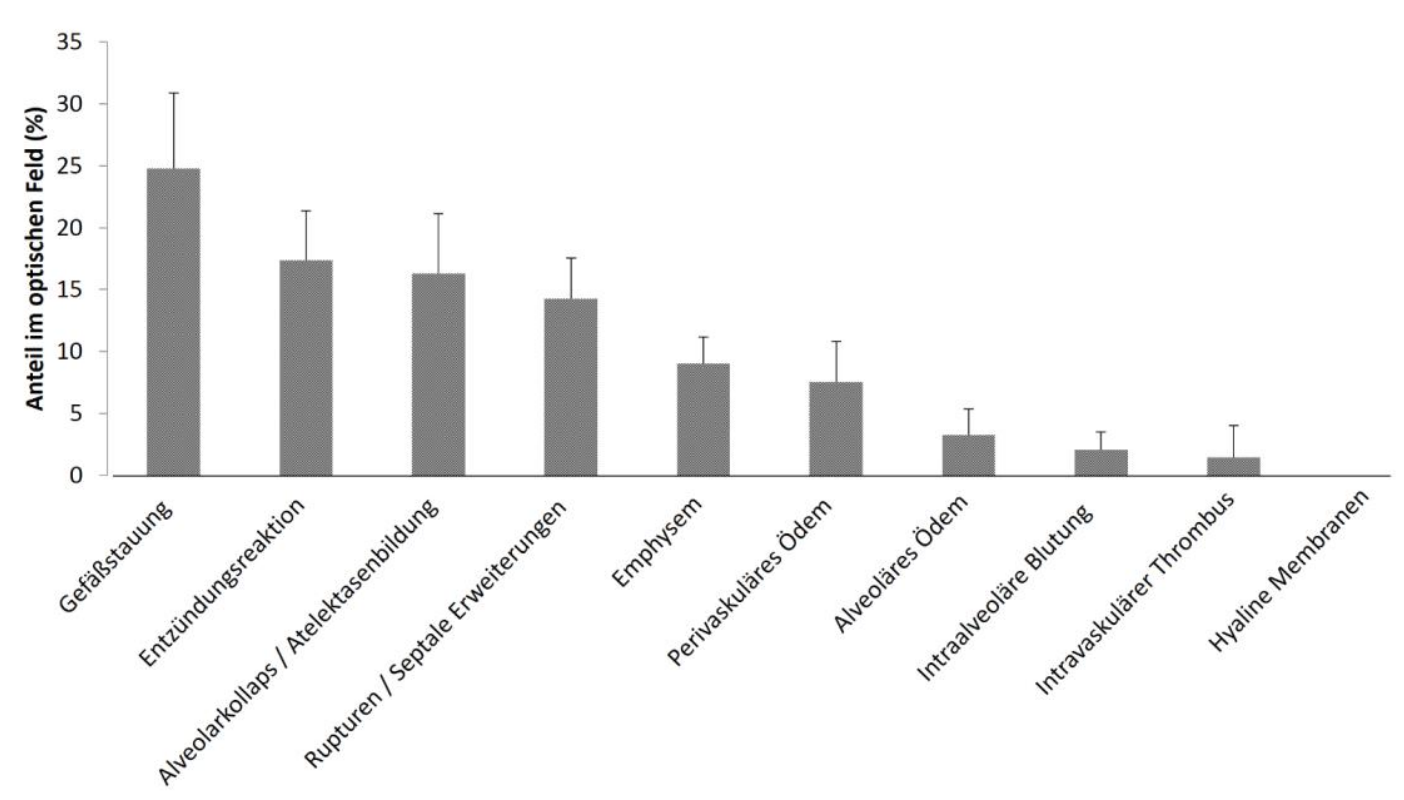

\section{Abbildung 17: Mikroskopischer Überblick}

Die aufgetretenen histopathologischen Veränderungen werden entsprechend ihres prozentualen Auftretens im optischen Feld in absteigender Häufigkeit als Säule gekennzeichnet. So wurden Gefäßstauungen, Entzündungsreaktionen, Alveolarkollapse/Atelektasenbildungen, Rupturen/septale Erweiterungen, Emphyseme, perivaskuläre Ödeme, alveoläre Ödeme, intraalveoläre Blutungen und intravaskuläre Thromben beobachtet und erfasst. Hyaline Membranen konnten nicht nachgewiesen werden. Die Verwendung der Abbildung erfolgt mit freundlicher Genehmigung des Wolters Kluwer Health Verlags (Collino et al. 2019). 

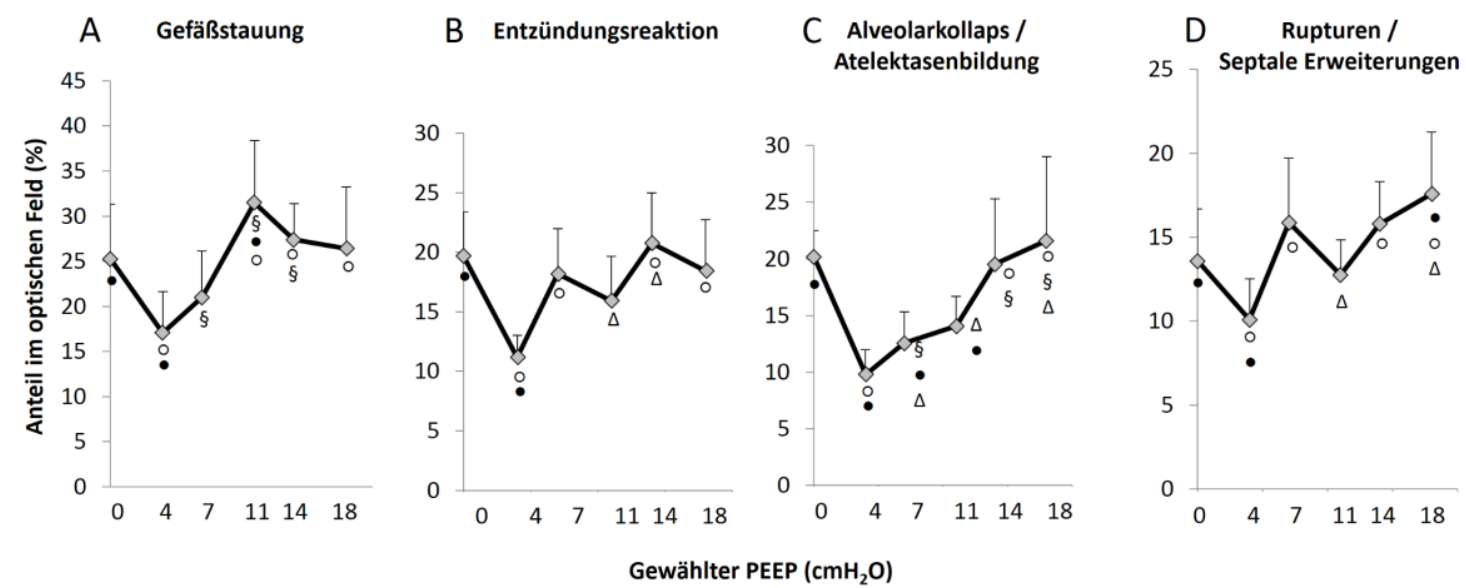

Abbildung 18: Verteilung der häufigsten mikroskopischen Läsionen nach PEEP-Gruppe

Die oben gezeigten Grafiken präsentieren die Häufigkeitsverteilung verschiedener mikroskopischer Läsionen entsprechend der jeweiligen PEEP-Gruppe, gemessen am prozentualen Anteil im optischen Feld. Dabei wurden Gefäßstauungen (A), Entzündungsreaktionen (B), Alveolarkollapse bzw. Atelektasenbildungen (C) und Rupturen/septale Erweiterungen (D) erfasst und untersucht. Signifikante statistische Unterschiede in Abgrenzung zu den anderen PEEP-Gruppen werden durch $\bullet$ für ZEEP, ○ für $4 \mathrm{cmH}_{2} 0, \S$ für $7 \mathrm{cmH}_{2} 0$ und $\Delta$ für $11 \mathrm{cmH}_{2} 0$ gekennzeichnet. Die Verwendung der Abbildung erfolgt mit freundlicher Genehmigung des Wolters Kluwer Health Verlags (Collino et al. 2019).

Das Verhalten der weniger häufigen strukturellen Veränderungen zeigt Abbildung 19. Darin wird deutlich, dass bei höheren PEEP-Werten (14-18 $\left.\mathrm{cmH}_{2} 0\right)$ im Vergleich zu niedrigen PEEP-Werten ein alveoläres Ödem unwahrscheinlicher und alveoläre Blutungen wahrscheinlicher auftraten. Hyaline Membranen wurden nicht beobachtet. 

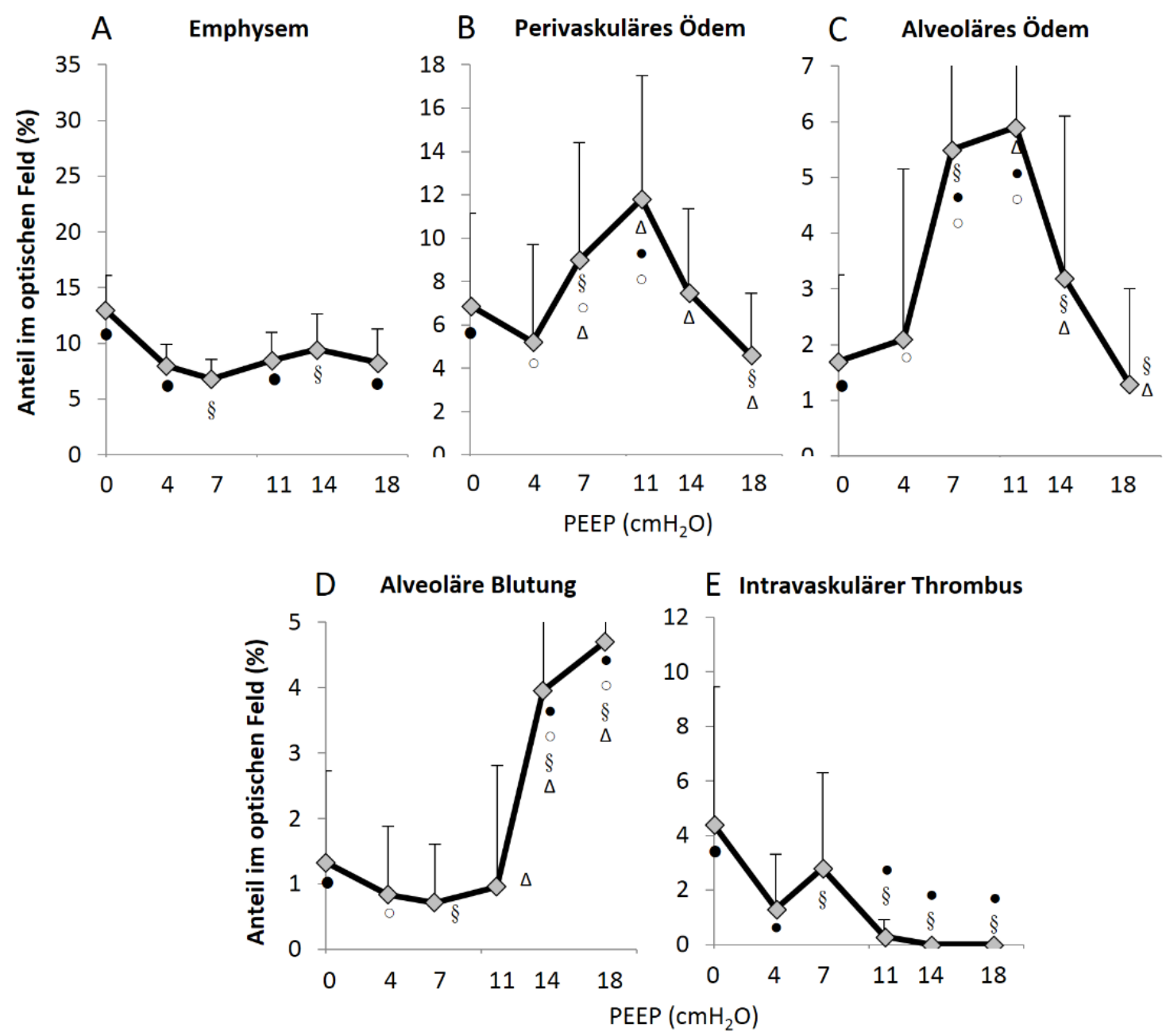

Abbildung 19: Verteilung der selteneren mikroskopischen Läsionen nach PEEP-Gruppen

Die oben gezeigten Grafiken präsentieren die Häufigkeitsverteilung verschiedener mikroskopischer Läsionen entsprechend der jeweiligen PEEP-Gruppe, gemessen am prozentualen Anteil im optischen Feld. Zu den untersuchten Lungenveränderungen zählen das Auftreten von Emphysemen (A), perivaskulären Ödemen (B), alveolären Ödemen (C), alveolären Blutungen (D) und intravaskulären Thromben (E). Signifikante statische Unterschiede in Abgrenzung zu den anderen PEEP-Gruppen werden durch $\bullet$ für ZEEP, ○ für $4 \mathrm{cmH}_{2} 0$, $\$ für $7 \mathrm{cmH}_{2} 0$ und $\Delta$ für $11 \mathrm{cmH}_{2} 0$ gekennzeichnet. Die Verwendung der Abbildung erfolgt mit freundlicher Genehmigung des Wolters Kluwer Health Verlags (Collino et al. 2019). 


\section{Tabelle 4: Lungengewicht und Wet-to-dry-Ratio}

Lungengewichte und Wet-to-dry-Verhältnisse werden in den verschiedenen PEEP-Gruppen als Mittelwert \pm Standardabweichung angegeben. Zu Vergleichszwecken wurde in der jeweils ersten Zeile ein Vergleich der PEEP-Gruppen mithilfe des ANOVA-Tests durchgeführt (dunkleres Grau). In der darunterliegenden Zeile erfolgt jeweils ein Vergleich zwischen niedriger und hoher PEEP-Gruppe mittels Student's t-Test (helleres Grau).

\begin{tabular}{|c|c|c|c|c|c|c|c|}
\hline PEEP & 0 & 4 & 7 & 11 & 14 & 18 & $\begin{array}{c}\text { P- } \\
\text { Wert }\end{array}$ \\
\hline & \multicolumn{3}{|c|}{ Niedriger PEEP } & \multicolumn{3}{|c|}{ Hoher PEEP } & \\
\hline \multirow{2}{*}{$\begin{array}{c}\text { Finales Lung- } \\
\text { engewicht / ini- } \\
\text { tiales } \\
\text { Schweinegewicht } \\
(\%)\end{array}$} & $\begin{array}{c}1,4 \pm 0,14 \\
(n=6)\end{array}$ & $\begin{array}{c}1,43 \pm 0,46 \\
(n=6)\end{array}$ & $\begin{array}{c}1,7 \pm 0,67 \\
(n=6)\end{array}$ & $\begin{array}{c}1,84 \pm 0,53 \\
(n=6)\end{array}$ & $\begin{array}{c}1,61 \pm 0,49 \\
(n=6)\end{array}$ & $\begin{array}{c}1,66 \pm 0,32 \\
(n=6)\end{array}$ & 0,562 \\
\hline & \multicolumn{3}{|c|}{$1,5 \pm 0,45$} & \multicolumn{3}{|c|}{$1,7 \pm 0,44$} & 0,18 \\
\hline \multirow{2}{*}{$\begin{array}{c}\text { Wet-to-dry-Index: } \\
\text { Lunge }\end{array}$} & $\begin{array}{c}5,9 \pm 0,3 \\
(n=6)\end{array}$ & $\begin{array}{l}6 \pm 0,7 \\
(n=6)\end{array}$ & $\begin{array}{c}6,6 \pm 0,7 \\
(n=6)\end{array}$ & $\begin{array}{c}6,5 \pm 0,4 \\
(n=6)\end{array}$ & $\begin{array}{l}6,8 \pm 0,9 \\
(n=6)\end{array}$ & $\begin{array}{c}10,3 \pm 4,8 \\
(n=6)\end{array}$ & 0,006 \\
\hline & \multicolumn{3}{|c|}{$6,2 \pm 0,6$} & \multicolumn{3}{|c|}{$7,9 \pm 3,2$} & 0,03 \\
\hline \multirow{2}{*}{$\begin{array}{c}\text { Wet-to-dry-Index: } \\
\text { Leber }\end{array}$} & $\begin{array}{c}3,9 \pm 0,3 \\
(n=3)\end{array}$ & $\begin{array}{c}3,7 \pm 0,2 \\
(n=3)\end{array}$ & $\begin{array}{c}3,6 \pm 0,3 \\
(n=4)\end{array}$ & $\begin{array}{c}4,4 \pm 0,5 \\
(n=3)\end{array}$ & $\begin{array}{c}4,8 \pm 1,5 \\
(n=4)\end{array}$ & $\begin{array}{c}7,0 \pm 4,2 \\
(n=4)\end{array}$ & 0,25 \\
\hline & \multicolumn{3}{|c|}{$3,7 \pm 0,3$} & \multicolumn{3}{|c|}{$5,5 \pm 2,7$} & 0,06 \\
\hline \multirow{2}{*}{$\begin{array}{c}\text { Wet-to-dry-Index: } \\
\text { Niere }\end{array}$} & $\begin{array}{c}4,7 \pm 1,2 \\
(n=3)\end{array}$ & $\begin{array}{c}4,3 \pm 1,1 \\
(n=3)\end{array}$ & $\begin{array}{c}4,9 \pm 0,8 \\
(n=4)\end{array}$ & $\begin{array}{c}5,4 \pm 0,2 \\
(n=3)\end{array}$ & $\begin{array}{c}6,0 \pm 1,4 \\
(n=4)\end{array}$ & $\begin{array}{c}9,5 \pm 4,5 \\
(n=4)\end{array}$ & 0,135 \\
\hline & \multicolumn{3}{|c|}{$4,7 \pm 0,9$} & \multicolumn{3}{|c|}{$7,1 \pm 3,6$} & 0,06 \\
\hline \multirow{2}{*}{$\begin{array}{c}\text { Wet-to-dry-Index: } \\
\text { Skelettmuskel }\end{array}$} & $\begin{array}{c}3,8 \pm 0,5 \\
(n=3)\end{array}$ & $\begin{array}{c}4,7 \pm 0,6 \\
(n=3)\end{array}$ & $\begin{array}{c}4,0 \pm 0,7 \\
(n=4)\end{array}$ & $\begin{array}{c}4,1 \pm 0,3 \\
(n=3)\end{array}$ & $\begin{array}{c}4,4 \pm 1,0 \\
(n=4)\end{array}$ & $\begin{array}{c}6,5 \pm 2,4 \\
(n=4)\end{array}$ & 0,09 \\
\hline & \multicolumn{3}{|c|}{$4,1 \pm 0,7$} & \multicolumn{3}{|c|}{$5,3 \pm 1,8$} & 0,14 \\
\hline \multirow{2}{*}{$\begin{array}{c}\text { Wet-to-dry-Index: } \\
\text { Darm }\end{array}$} & $\begin{array}{c}5,5 \pm 0,5 \\
(n=3)\end{array}$ & $\begin{array}{c}5,6 \pm 0,3 \\
(n=3)\end{array}$ & $\begin{array}{c}5,6 \pm 0,9 \\
(n=4)\end{array}$ & $\begin{array}{c}5,2 \pm 0,2 \\
(n=3)\end{array}$ & $\begin{array}{c}6,0 \pm 1,4 \\
(n=4)\end{array}$ & $\begin{array}{c}8,5 \pm 4,2 \\
(n=4)\end{array}$ & 0,31 \\
\hline & \multicolumn{3}{|c|}{$5,6 \pm 0,6$} & \multicolumn{3}{|c|}{$6,7 \pm 2,8$} & 0,24 \\
\hline
\end{tabular}




\subsubsection{Lungenödem: Lungengewicht und Wet-to-dry-Ratio}

Tabelle 4 zeigt das relative Gewicht der Lunge am Ende des Experiments in Bezug auf das ursprüngliche Gewicht des Schweins und die Wet-to-dry-Ratio-Index-Werte (W/D-Index) aus Proben der Lunge, des Darms, der Leber, der Niere sowie des Skelettmuskels.

Die Index-Werte der untersuchten Organe waren in den Gruppen mit einem höheren PEEP $(11,14,18)$ höher als in denen mit niedrigerem PEEP, wobei dieser Unterschied nur für die Lunge statistisch signifikant erschien (s. Abb. 20). Dabei ist zu beachten, dass für die anderen Organe nur eine geringere Anzahl von Proben zur Verfügung stand.

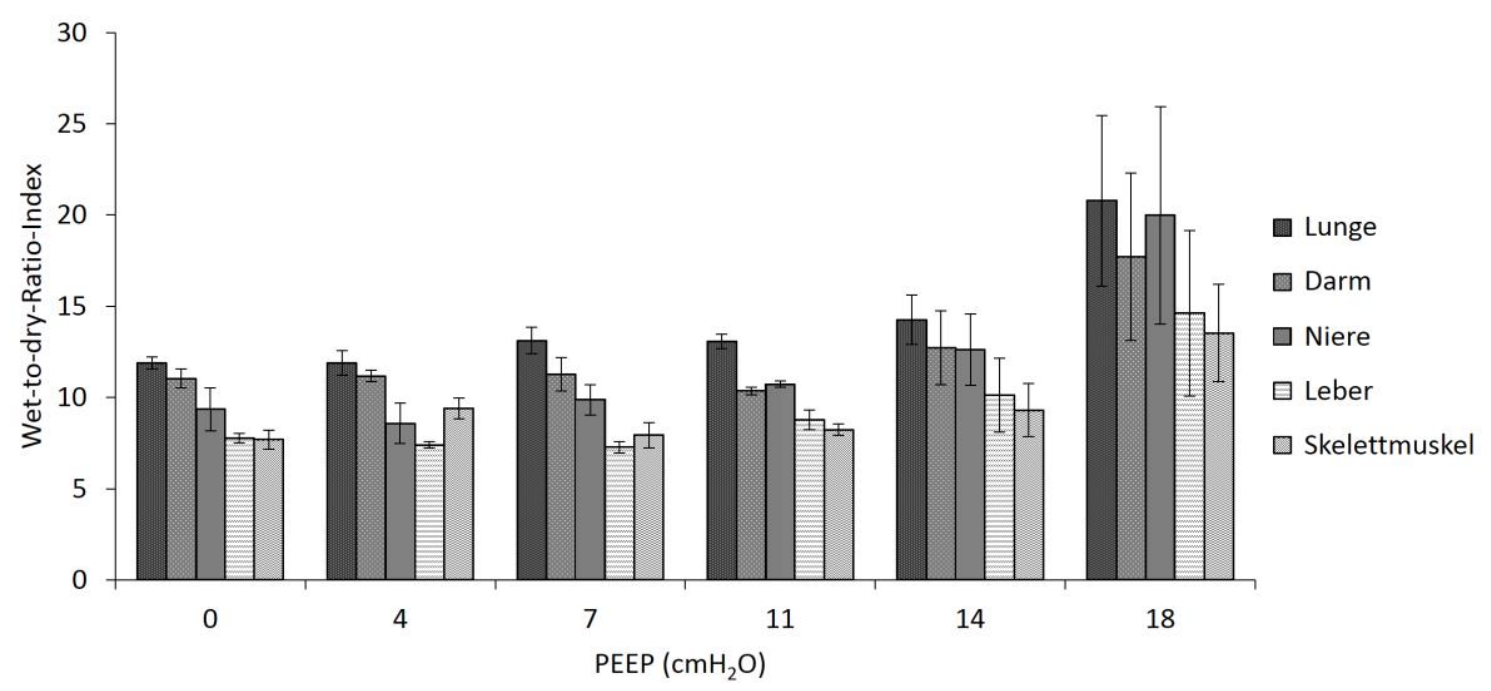

\section{Abbildung 20: Wet-to-dry-Ratio-Index in den verschiedenen Organsystemen}

Der Wet-to-dry-Ratio-Index wird in den unterschiedlichen PEEP-Gruppen in die fünf Organe: Lunge, Darm, Niere, Leber und Skelettmuskel jeweils durch Säulen in unterschiedlichen Graustaufen (entsprechend der Legende in der Abbildung) dargestellt. Dazu sind die Standardabweichungen angegeben. Die Verwendung der Abbildung erfolgt mit freundlicher Genehmigung des Wolters Kluwer Health Verlags (Collino et al. 2019). 


\subsection{PEEP und die Grenzwerte der mechanischen Leistung}

Ausgewählte potenzielle Surrogatparameter für einen Lungenschaden sind in Tabelle 5 repräsentiert. Die Datensätze aller Schweine - ungeachtet ihrer PEEP-Gruppen-Zugehörigkeit - wurden zusammengeführt und der jeweilige Median bestimmt. Um einen möglichen Grenzwert für die mechanische Leistung zu identifizieren, wurden mithilfe der Mediane dieser bestimmten Variablen ROC-Kurven für die jeweiligen Parameter erstellt und ein Modell einer logistischen Regression kreiert. Eine Mittelwertbildung dieser gewonnenen Schwellenwerte für die mechanische Leistung suggerieren einen Grenzwert für die auf die Lunge applizierte Leistung von $13 \pm 1 \frac{\mathrm{J}}{\mathrm{min}}$ und für das respiratorische System von $25 \pm 1,7 \frac{\mathrm{J}}{\mathrm{min}}$. Für weitere Details sei auf die Veröffentlichung unserer Arbeitsgruppe verwiesen (Collino et al. 2019). 
Tabelle 5: Mögliche Grenzwerte der mechanischen Leistung

Zusammenfassung der Ergebnisse der receiver operating characteristic (ROC)-Kurven, welche durch die Median-Werte zahlreicher Variablen als cut-off-Werte erstellt wurden, um so diejenige mechanische Leistungsgrenze zu ermitteln, welche am besten zwischen leichten und schwerwiegenden Schäden unterscheiden kann. (Abkürzungen: AUC: area under the curve (Fläche unter der Kurve/Integral); CI: confidence interval (Vertrauensintervall)). Für weitere Details sei auf die Veröffentlichung unserer Arbeitsgruppe verwiesen.

\begin{tabular}{|c|c|c|c|c|c|}
\hline Variable & Median & $\begin{array}{l}\text { Mechanische } \\
\text { Leistung: } \\
\text { Lunge } \\
\text { (J/min) }\end{array}$ & $\begin{array}{l}\text { AUC } \\
(\text { ROC) \& } \\
\text { CI }\end{array}$ & $\begin{array}{l}\text { Mechanische } \\
\text { Leistung: Respira- } \\
\text { torisches System } \\
\text { (J/min) }\end{array}$ & $\begin{array}{l}\text { AUC } \\
\text { (ROC) \& } \\
\text { CI }\end{array}$ \\
\hline Wet-to-dry lung index & 6,45 & 12,1 & $\begin{array}{l}0,78 \\
(0,61-0,92)\end{array}$ & 22,7 & $\begin{array}{l}0,71 \\
(0,53-0,88)\end{array}$ \\
\hline Pathologie-Score & 97 & 13 & $\begin{array}{l}0,59 \\
(0,4-0,78)\end{array}$ & 27,9 & $\begin{array}{l}0,58 \\
(0,39-0,77)\end{array}$ \\
\hline $\begin{array}{l}\text { Elastance der Lunge } \\
\left(\mathrm{cmH}_{2} \mathrm{O} / \mathrm{L}\right)\end{array}$ & 28,6 & 13,2 & $\begin{array}{l}0,84 \\
(0,8-0,88)\end{array}$ & 25,8 & $\begin{array}{l}0,69 \\
(0,63-0,75)\end{array}$ \\
\hline $\begin{array}{l}\text { Spezifische Elastance } \\
\text { der Lunge }\left(\mathrm{cmH}_{2} \mathrm{O} / \mathrm{L}\right)\end{array}$ & 10,1 & 13,1 & $\begin{array}{l}0,9 \\
(0,87-0,93)\end{array}$ & 25,3 & $\begin{array}{l}0,8 \\
(0,75-0,84)\end{array}$ \\
\hline Totraum (\%) & 40 & 13,4 & $\begin{array}{l}0,62 \\
(0,56-0,68)\end{array}$ & 25,6 & $\begin{array}{l}0,57 \\
(0,5-0,63)\end{array}$ \\
\hline $\begin{array}{l}\text { Treibende Druckdiffe- } \\
\text { renz } \\
\left(\mathrm{cmH}_{2} \mathrm{O}\right)\end{array}$ & 16,4 & 13 & $\begin{array}{l}0,91 \\
(0,87-0,94)\end{array}$ & 25,3 & $\begin{array}{l}0,84 \\
(0,8-0,88)\end{array}$ \\
\hline $\begin{array}{l}\text { PEEP } \\
\left(\mathrm{cmH}_{2} \mathrm{O}\right)\end{array}$ & 8,7 & 11,7 & $\begin{array}{l}0,91 \\
(0,88-0,94)\end{array}$ & 22,0 & $\begin{array}{l}0,91 \\
(0,88-0,94)\end{array}$ \\
\hline $\begin{array}{l}\text { Transpulmonale trei- } \\
\text { bende Druckdifferenz } \\
\left(\mathrm{cmH}_{2} \mathrm{O}\right)\end{array}$ & 8,12 & 15,51 & $\begin{array}{l}0,76 \\
(0,71-0,8)\end{array}$ & 25,16 & $\begin{array}{l}0,64 \\
(0,58-0,69)\end{array}$ \\
\hline $\begin{array}{l}\text { Mittlerer pulmonalarte- } \\
\text { rieller Druck } \\
(\mathrm{mmHg})\end{array}$ & 23 & 12,6 & $\begin{array}{l}0,86 \\
(0,82-0,90)\end{array}$ & 24,9 & $\begin{array}{l}0,87 \\
(0,81-0,93)\end{array}$ \\
\hline Mittelwert & & $13 \pm 1$ & & $25 \pm 1,7$ & \\
\hline
\end{tabular}




\section{Diskussion}

Um den Einfluss unterschiedlich hoher PEEP-Werte bei der maschinellen Beatmung auf einen sich entwickelnden Lungenschaden tierexperimentell zu untersuchen wurden 40 weibliche Schweine über einen Zeitraum von 50 Stunden maschinell beatmet. Dabei möchte ich der Frage nachgehen, welche Auswirkungen ein PEEP auf den Körper hat. Dazu werde ich zunächst die lokalen Veränderungen auf die Lunge unter Berücksichtigung des Konzeptes der mechanischen Leistung erörtern und anschließend die untersuchten systemischen Veränderungen wie beispielsweise die Hämodynamik näher erläutern. Daraus abgeleitet ist die Suche nach einem besonders geeigneten Surrogatparameter für den Lungenschaden zentrales Thema dieser Dissertation. Dabei gilt es zu untersuchen, ob das Konzept der mechanischen Energie, welches bereits tierexperimentell untersucht wurde, um die PEEP-Komponente erweitert werden kann. Die statische strain-Komponente durch den PEEP stellt eine einmalige Energieübertragung im Sinne potentieller Energie dar, wohingegen die dynamische strain-Komponente, welche sich durch das Tidalvolumen ergibt, atemzyklisch appliziert wird, sodass sie mehr einer kinetischen Energie entspricht (Protti et al. 2015). Außerdem wirken, wie bereits in der Herleitung der Formel der mechanischen Leistung gezeigt, die Atemfrequenz (Hotchkiss, JR et al. 2000; Rich et al. 2000; Rich et al. 2003) und der Inspirationsfluss (Fujita et al. 2007) eine entscheidende Rolle in Bezug auf die mechanische Leistung und damit verbunden auch bei der Entstehung einer VILI. Aber womöglich sind auch noch nicht erfasste Parameter wie beispielsweise die Körperkerntemperatur relevant in Bezug auf die Pathogenese (Suzuki et al. 2004).

Bei Schweinen entwickelt sich scheinbar eine VILI, wenn durch die mechanische Leistung

ein Grenzwert von $12 \frac{\mathrm{J}}{\mathrm{min}}$ überschritten wird (Protti et al. 2016). Bis zu diesem Schwellenwert zeigen sich in CT-Aufnahmen, nur vereinzelte (vermutlich stress raiser-bedingte) Verdichtungen (Protti et al. 2016). Wird dieser Grenzwert überschritten, zeigen sich weitläufige Ödemanzeichen. Diese Studie von Cressoni et al. wurde allerdings ohne appliziertem PEEP durchgeführt und so blieb die Frage offen, inwieweit ein PEEP die Entwicklung der mechanischen Leistung beeinflusst. In diesem Experiment konnte gezeigt werden, dass der PEEP - wie theoretisch oben geschildert - einen erheblichen Anteil zur mechanischen Leistung beiträgt (Cressoni et al. 2016). 
Die Versuchstiere wurde in sechs Gruppen mit PEEP-Werten 0, 4, 7, 11, 14 und $18 \mathrm{~cm} \mathrm{H}_{2} 0$ eingeteilt, sodass überprüft werden kann, ob die isolierte Erhöhung des PEEP ein Ergotrauma auslösen, verstärken oder anderweitig beeinflussen kann, um letztlich zu klären, ob der PEEP, wie weitläufig angenommen, protektiv oder schädlich ist. Dazu werden atemmechanische Parameter (wie die mechanische Leistung) analysiert, um einen möglichen Grenzwert der mechanischen Leistung für das Auftreten eines beatmungsgerätassoziierten Lungenschadens zu definieren, welcher den PEEP berücksichtigt. Dabei sollten pathologische Untersuchungen und die Entwicklung von Flüssigkeitsansammlungen in der Lunge als Marker für eine aufgetretene Beschädigung der Lunge herangeführt, aber auch kritisch beleuchtet werden. Bei der Patientenanwendung könnte mithilfe des Konzepts der mechanischen Leistung und deren Grenzwert in Zukunft ein sicheres Kriterium entwickelt werden, bis wann eine bestimmte Konstellation maschineller Beatmungsparameter unter Berücksichtigung individueller Lungeneigenschaften eine Lungenprotektion gewährleisten kann.

\subsection{Der Einfluss des PEEP auf die mechanische Leistung}

In dieser Studie wurden die Tiere der ZEEP-Gruppe einer signifikant höheren dynamischen mechanischen Leistung ausgesetzt als diejenigen Tiere mit einem PEEP von 4 oder $7 \mathrm{~cm} \mathrm{H}_{2} \mathrm{O}$ (s. Abb. 5). Ein Lungenschaden manifestierte sich dabei durch eine verringerte FRC, erhöhte Atelektasenbildung, inflammatorische Infiltrationen sowie eine gesteigerte Lungenelastance und spezifische Elastance (Collino et al. 2019). Die Tiere der ZEEP-Gruppe wiesen außerdem das geringste Lungengewicht, den niedrigsten W/D-Index sowie Noradrenalin-Bedarf und verbrauchte Volumensubstitution auf, wohingegen der mittlere pulmonal-arterielle Druck und der Wedge-Druck denen der PEEP-Gruppe von 4 bzw. $7 \mathrm{~cm} \mathrm{H}_{2} 0$ ähnelten (Collino et al. 2019) (vgl. Tab. 1 und 3). Die von uns ermittelten Daten legen den Schluss nahe, dass bei ZEEP ein Atelektrauma und eine erhöhte dynamische Leistung die relevantesten auslösenden Faktoren eines Lungenschadens sind, welcher weitestgehend ohne hämodynamische Konsequenzen auftritt (Collino et al. 2019).

Die gesamte mechanische Leistung war bei einem PEEP von 0, 4 und $7 \mathrm{~cm} \mathrm{H}_{2} \mathrm{O}$ ähnlich, allerdings waren bei einem PEEP von 4 und $7 \mathrm{~cm} \mathrm{H}_{2} \mathrm{O}$ die dynamische Komponente der Leistung signifikant um etwa 35\% reduziert, während die statische Komponente im Vergleich zu ZEEP dafür 3 bzw. 6 Mal größer war (Collino et al. 2019) (s. Abb. 5). 
Lungenelastance, spezifische Lungenelastance, Atelektasenbildung und Entzündungsreaktionen waren signifikant niedriger in der PEEP $4 \mathrm{~cm} \mathrm{H}_{2} \mathrm{O}$-Gruppe im Vergleich zur ZEEPGruppe, während die Werte relativ ähnlich waren zwischen der PEEP 4 und $7 \mathrm{~cm} \mathrm{H}_{2} \mathrm{O}$ (vgl. Tab. 1 und 2). Darin scheint sich zumindest in Bezug auf das Atelektrauma ein gewisser protektiver Effekt zu bestätigen. Der kleine, aber signifikante Anstieg des strain, des Totraums, der Volumensubstitution und des pulmonal-arterieller Blutdrucks, welche bei einem PEEP von $7 \mathrm{~cm} \mathrm{H}_{2} \mathrm{O}$ im Vergleich zu einem PEEP von $4 \mathrm{~cm} \mathrm{H}_{2} \mathrm{O}$ zu beobachten sind, suggeriert, dass selbst ein kleiner PEEP-Anstieg von $3 \mathrm{~cm} \mathrm{H}_{2} \mathrm{O}$ ein bedeutsamen Einfluss auf die Lungenausdehnung und die hämodynamischen Parameter in den zuvor gesunden Versuchstieren hat (vgl. Tab. 3) (Collino et al. 2019). Die Tatsache, dass der größte Schaden in der ZEEP-Gruppe nachgewiesen wurde, scheint die Annahme zu bekräftigen, dass unterhalb einer bestimmten Grenze die dynamische Komponente der mechanischen Leistung womöglich ein größeres schädliches Potential als oberhalb davon aufweist (Collino et al. 2019).

Dies steht auch im Einklang mit den Ergebnissen früherer Experimente, die gezeigt haben, dass nicht nur der Wert des globalen strain die Auswirkungen auf das Lungengewebe bestimmt, sondern auch die Anteile seiner statischen (auf Grund des PEEP) und dynamischen Komponenten (auf Grund der treibenden Druckdifferenz $\left(P_{\text {plat }}-P E E P\right)$ ) (Protti et al. 2013). In-vitro-Versuche stützen diese Überlegung, denn Alveolar-Epithel-Zellen nehmen eher durch zyklische als durch tonische Verformung bei gleicher maximaler Ausdehnung Schaden (Tschumperlin et al. 2000; Ye et al. 2012).

Eine weitere Erhöhung des PEEP auf Werte wie 11, 14 oder $18 \mathrm{~cm} \mathrm{H}_{2} 0$ führt zu einer mechanischen Leistung auf die Lungen, welche auf Grund der größeren statischen Leistungskomponente oberhalb der angenommenen kritischen Schwelle liegt (vgl. Abb. 5). Dahingegen entspricht die dynamische Leistungskomponente bei diesen PEEP-Werten der der ZEEP-Gruppe. Aufgetretene Schäden in der ZEEP-Gruppe, welche sich im Lungengewicht, histologischen Atelektasenbildung und inflammatorischen Infiltrationen widerspiegeln, wurden in den Gruppen mit höheren PEEP-Werten scheinbar nicht vorgebeugt (vgl. Abb. 18-20) (Collino et al. 2019). Andere Indikatoren wie stress, strain, Lungenelastance und die spezifische Lungenelastance wurden sogar signifikant um 40-70 \% erhöht und implizieren somit einen Lungenschaden, welcher durch einen erhöhten PEEP aufgetreten ist (vgl. Tab. 1) (Collino et al. 2019). Außerdem sind weitere Veränderungen nur bei diesen höheren 
PEEP-Werten aufgetreten: Eine Erhöhung des mittleren pulmonal-arteriellen Drucks (um 40 \%), zusätzliche Volumensubstution (um $145 \%$ ) und die erhöhte Notwendigkeit einer Noradrenalin-Infusion (um 100 \%) (vgl. Tab. 3).

Letztlich waren die sechs vorzeitigen Tode der Versuchstiere aus den PEEP 14 und 18Gruppe klar entweder einem hämodynamischen (fünf Tiere) oder einem respiratorischen Versagen (durch einen Spannungspneumothorax; ein Tier) zuzuordnen (vgl. Abb. 7) (Collino et al. 2019). Eine Erhöhung des PEEP über Werte von $7 \mathrm{cmH}_{2} \mathrm{O}$ hat also nicht nur keinen Lungenschaden vorgebeugt, sondern zusätzlich eine Belastung durch hämodynamische Instabilität geschaffen, welche das Risiko für eine Schädigung oder ein frühzeitiges Ableben signifikant erhöht (Collino et al. 2019).

Allerdings zeigt der Vergleich der verschiedenen PEEP-Gruppen in unserem Experiment, dass bei steigendem PEEP-Level sich das Schadensausmaß von einer Lungenödementwicklung (durch das Atelektrauma) hin zu einer Überblähung (durch ein Volutrauma) verschiebt. Ursache dafür könnte das Wechselspiel von PEEP und den daraus resultierenden hämodynamischen Konsequenzen und die sich daraus ergebenden ins Lungengewebe gerichteten Filtrationsdrücke sein (Collino et al. 2019).

\subsection{Mechanische Leistung und PEEP im zeitlichen Verlauf}

Eine beschädigte Lunge weist ein verringertes ventilierbares Volumen durch die Verminderung der offenen Atemwege und kollabierte Alveolen auf, welches zu einer Zunahme der benötigten Drücke und Flüsse und dadurch insgesamt zu einer erhöhten mechanischen Leistung führt, um ein konstantes Tidalvolumen zu erreichen (Cressoni et al. 2016). Diese erhöhte mechanische Leistung resultiert in einer verstärkten Zunahme der VILI und erklärt so möglicherweise zu einem gewissen Teil den exponentiellen Verlauf einer VILI (Cressoni et al. 2016; Cressoni et al. 2015). 


\subsection{PEEP und Gasaustausch}

Obwohl die Lungenstruktur in den meisten PEEP-Gruppen schwerwiegende Zerstörungen aufweist, zeigte der Gasaustausch keine relevanten Schäden (vgl. Tab. 2). Alle Oxygenierungsvariablen und zum Teil die $\mathrm{CO}_{2}$-Parameter waren normwertig (vgl. Tab. 2). Dies resultiert aus den speziellen Bedingungen in diesem Experiment. Denn dem Kollabieren der Alveolen wird durch den hohen PEEP, dem hohen Tidalvolumen, den hohen mittleren Atemwegsdruck der Reduktion des Herz-Zeit-Volumens vorgebeugt, indem diese Komponenten dazu beitragen kein Shunt-Volumen aufkommen zu lassen und folglich keine unphysiologische Reduktion des Ventilations-Perfusions-Verhältnis hervorzurufen (vgl. Tab. 3). Dass die diesbezüglich schlechtesten Werte für die Schweine der ZEEP-Gruppen zu finden sind, stützt diese Überlegungen. Aus diesem Grund reihen sich unsere Beobachtungen in die klinischen und experimentellen Erkenntnisse der letzten Dekaden ein, dass hohe Beatmungsvolumina und -drücke für gewöhnlich mit einer verbesserten Oxygenierung einhergehen (Gattinoni et al. 2017b; Collino et al. 2019).

\subsection{PEEP und Hämodynamik}

Das hämodynamische Verhalten resultiert aus dem Zusammenspiel von Herzaktion und dem übersteigerten Druck und Volumen, welche der Lunge durch das Beatmungsgerät aufgezwungen werden. Dies zeigt sich zum Beispiel darin, dass das Herz-Zeit-Volumen bei zunehmenden PEEP-Werten abnimmt und alle Drücke im Pulmonalkreislauf anstiegen (vgl. Tab. 3) (Collino et al. 2019). Die notwendige Flüssigkeitssubstitution sowie die NoradrenalinDosis steigt signifikant mit dem verwendeten PEEP (vgl. Tab. 3).

Das Auftreten einer Stauung dem Herzen vorgeschalteter Organe könnte eine pathogenetische Rolle spielen. Eine so möglicherweise diffus auftretende Verschlechterung dieser Organe wird im Experiment durch die W/D-Ratio in Leber, Darm, Skelettmuskel und Niere näherungsweise erfasst (vgl. Tab. 4) (Collino et al. 2019). Allerdings zeigte sich in diesem Experiment keine signifikanten Abweichungen, was vermutlich am geringen Stichprobenumfang lag. 


\subsection{Ein Grenzwert der mechanischen Leistung}

Frühere Experimente konnten zeigen, dass ventilationsinduzierte Lungenschäden in lungengesunden Schweinen sich nicht unbegrenzt proportional zum applizierten strain und stress entwickeln, sondern nur dann, wenn ein strain von über 1,5-2 erreicht oder sogar überschritten wird. Oberhalb dieses Grenzwertes endet die Linearität der strain-stress-Beziehung und geht in einen exponentiellen Verlauf über. Dieser Übergang scheint der Punkt zu sein, an dem Lungenregionen ihre anatomischen und physiologischen Grenzen in Form ihrer Totalkapazität erreichen (Protti et al. 2011).

Beim Überschreiten dieses möglichen Grenzwertes könnten die chemischen Bindungen der extrazellulären Matrix bildenden Polymere zerstört werden (Parker et al. 1997; Al-Jamal und Ludwig 2001; Pelosi und Rocco 2008) und so kontinuierlich zu einer Art Materialermüdung führen (Gattinoni et al. 2003). Durch zyklische Applikation einer mechanischen Leistung oberhalb eines Schwellenwertes kommt es daraufhin zu Mikrofrakturen, wodurch die Produktion niedermolekularer Hyaluronane induziert wird, welche Auslöser für eine inflammatorische Reaktion (Jiang et al. 2005) und Reparaturmechanismen darstellen (Jiang et al. 2005; Pelosi und Rocco 2008).

In diesem Experiment wurde also eine mögliche Schwelle der mechanischen Leistung von etwa $13 \frac{\mathrm{J}}{\mathrm{min}}$ bestimmt, welche die anatomischen, mechanischen und hämodynamischen Schadensvariabeln im Bereich ihrer Mittelwerte aufteilt (vgl. Tab. 5) (Collino et al. 2019). Das ist relativ kongruent mit vorherigen experimentellen Ergebnissen, bei denen die mechanische Leistung dadurch verändert wurde, dass die Beatmungsfrequenz variiert wurde und das Tidalvolumen bei ZEEP konstant gehalten wurde (12,5 $\frac{\mathrm{J}}{\mathrm{min}}$ ) (Cressoni et al. 2016). Dabei soll hervorgehoben werden, dass ein solcher Grenzwert bei der maschinellen Beatmung als oberes Limit vor dem Auftreten tödlicher Schäden und nicht als Schwelle, unter welcher kein Schaden auftreten kann, verstanden werden sollte (Collino et al. 2019). Zumal in allen PEEPGruppen ein Lungenschaden nachgewiesen wurde (vgl. Abb. 18 und 19). Womöglich trat dies auf, weil die verwendeten Beatmungsparameter ungeachtet des applizierten PEEP-Wertes für sich genommen schädlich waren (Collino et al. 2019). 


\subsection{Abschätzung einer VILI}

Eins der Hauptprobleme im Zusammenhang mit der maschinellen Beatmung ist das Abschätzen der Wahrscheinlichkeit, dass eine beatmungsgerät-assoziierten Lungenschädigung auftritt. Eine VILI umfasst eine Vielzahl von anatomischen, physiologischen und klinischen Zeichen (Kumar et al. 1973; Dreyfuss et al. 1988). Experimentell sind ein erhöhtes Lungengewicht (Lungenödembildung), Shunt, Totraum und Elastance sowie ein histologischer Nachweis von Atelektasen und inflammatorische Infiltrationen die häufigsten Erscheinungen eines Atelektraumas (Dreyfuss et al. 1988). Das typische Merkmal eines Volutraumas ist hingegen die Überblähung (Hyperinflation) mit den jeweiligen Effekten auf Lungenmechanik und Hämodynamik (Collino et al. 2019; Dreyfuss et al. 1988). In diesem Experiment manifestierte sich als Ausdruck eines Schadens bei ZEEP vor allem das Atelektrauma, obwohl das Lungenödem kein wichtiges Merkmal war, womöglich, weil ein höherer mittlerer Atemwegsdruck dies verhindert hat (Colmenero-Ruiz et al. 1997; Myers et al. 1988; Russell et al. 1982). Bei einem im Vergleich dazu erhöhten PEEP verschiebt sich das Schädigungsmuster hin zu einem Volutrauma mit den typischen Konsequenzen für Lungenmechanik, Hämodynamik und Mortalität, aber ohne relevante Verschlechterungen in Bezug auf Lungenödembildung und Gasaustausch (Collino et al. 2019).

In klinischen Studien wird die vermeintliche Zunahme oder Abnahme einer VILI durch die Zu- oder Abnahme der Mortalität gemessen (Brower et al. 2000; Cavalcanti et al. 2017; Meade et al. 2008; Brower et al. 2004; Briel et al. 2010; Mercat et al. 2008), obwohl die Mortalität, welche allein der maschinellen Beatmung zugeschrieben werden kann, klinisch schwierig zu erfassen ist. Im Gegensatz dazu konnten in diesem Modell alle negativen Auswirkungen, einschließlich der Mortalität, auf die zu Beginn gesunden Schweine auss chließlich den Folgen der maschinellen Beatmung zugeschrieben werden (Collino et al. 2019). Dabei sollte betont werden, dass ein direkter Vergleich von ARDS-vorgeschädigten Lungen im klinischen Alltag und dem experimentellen gesunden Schweine-Lungen Einschränkungen unterliegt. 


\subsection{Limitationen}

\subsubsection{Einschränkungen im Studiendesign}

In diesem Experiment sollte die Rolle des PEEP als Komponente der mechanischen Leistung untersucht werden. Leider ist es nicht möglich (ohne künstliche Lungen) die mechanische Leistung durch den PEEP zu erhöhen, ohne dabei einige Änderungen an den anderen Beatmungsparametern vorzunehmen (Collino et al. 2019). In unserem Modell, wenn wir ein konstantes Tidalvolumen und eine konstante Beatmungsfrequenz wählen, führt ein erhöhter PEEP zwangsläufig entweder zu einem linearen Anstieg des Plateaudrucks und der treibenden Druckdifferenz entlang des mittleren geradlinigen Abschnitts der Ruhedehnungskurve oder zu einem exponentiellen Anstieg im oberen Abschnitt der Ruhedehnungskurve (Collino et al. 2019). Das Problem dabei ist allerdings nicht, ob der der PEEP, oder das Tidalvolumen oder die treibende Druckdifferenz oder die Beatmungsfrequenz jeweils einen stärkeren Einfluss auf das Auftreten einer VILI haben, sondern sie als Einheit zu verstehen, weil eine VILI, wie bereits gezeigt wurde, von all diesen Parametern bei der maschinellen Beatmung abhängig ist (Collino et al. 2019). Es erscheint daher logisch, dass die mechanische Leistung eine Vereinheitlichung darstellt (Collino et al. 2019). Der PEEP kann dabei der entscheidende Faktor für die Entstehung von Schäden in einigen Situationen sein, wie beispielsweise dieses Experiment zeigen konnte, wohingegen in anderen Situationen es vielleicht hauptsächlich von der treibenden Druckdifferenz (Amato et al. 1998) oder der Beatmungsfrequenz (Cressoni et al. 2016) oder deren Kombination (Ferguson et al. 2013) abhängig sein könnte (Collino et al. 2019). 


\subsubsection{Einschränkungen des Konzepts der mechanischen Leistung}

Unser Experiment hat dabei allerdings einige Einschränkungen, da es einige grundlegende Fragen in Bezug auf die mechanische Leistung nicht beantworten kann.

Erstens, wie bereits diskutiert, kann ein Anstieg des PEEP bei gleichbleibenden Tidalvolumen zu einem Anstieg des Plateaudrucks und der treibenden Druckdifferenz führen, sodass eine Interpretation daraus resultierender Ergebnisse willkürlich erscheinen kann (Collino et al. 2019).

Zweitens ist es sehr schwierig die bestmögliche Normierung der mechanischen Leistung durchzuführen (Collino et al. 2019). Thermodynamisch betrachtet, empfiehlt es sich intensive Größen (Zustandsgrößen, welche bei unterschiedlichen Größen des betrachteten Systems keine Änderung erfahren (Wedler 1997)) wie das Tidalvolumen zu skalieren, um mithilfe des strains so vergleichbare Aussagen auch zu pathologisch veränderten Lungen wie einer ARDS-baby-lung zu treffen (Protti et al. 2015; Gattinoni and Pesenti 2005). So wurde das Tidalvolumen in diesem Experiment verändert, um letztlich je nach FRC den PlateauDruck in gewissen Grenzen zu halten. Die treibende Druckdifferenz war somit relativ ähnlich in den verschiedenen PEEP-Gruppen, sodass diese allein (ohne PEEP) Schäden vermutlich nicht allein erklären kann. Die treibende Druckdifferenz war bei ZEEP sehr ähnlich zu der bei einem gewählten PEEP von 11, 14 und $18 \mathrm{~cm} \mathrm{H}_{2} \mathrm{O}$, obwohl die Schäden dramatisch und progressiv zunahmen (inklusive der Mortalität). Ebenso die extensiven Größen (Zustandsgrößen, welche bei unterschiedlichen Größen des betrachteten Systems Änderungen erfahren (Wedler 1997)) wie der Atemwegsdruck können je nach Verhältnis von Lungenzu Thoraxelastance unterschiedliche transpulmonale Drücke (stress) hervorrufen (Protti et al. 2015; Chiumello et al. 2008a). Aber es erscheint schwierig die mechanische Leistung, welche mehrere Komponenten umfasst auf eine dieser Größen zu skalieren. In diesem Tierexperiment ist eine solche Normierung nicht zwingend erforderlich, da eine studieninhärente Normierung durch die Auswahl gesunder Schweine gleichen Alters und Gewicht erfolgte. Eine systemische Normierung wäre bei einem Vergleich verschiedener Spezies allerdings unabdingbar (Collino et al. 2019). Um beispielsweise speziesübergreifende Aussagen zum strain zu treffen, wird das Verhältnis aus inspiratorischer Kapazität (TLC-FRC) zu FRC verglichen und dabei ergeben sich ähnliche Werte für Schweine (2,6 \pm 0,6), Mäuse (2-2,3), Ratten (2-3) und Menschen (2,2) (Protti et al. 2015), wodurch die physiologische maximale Ausdehnung 2-3 mal der FRC entspricht, also ein strain von 2-3. Der strain-Schwellenwert für eine tödliche 
Beatmung über die verschiedenen Spezies beträgt etwa 2,6 (Caironi et al. 2011). Der notwendige transpulmonale Druck (stress), um die Lunge auf die 2-fache FRC auszudehnen, entspricht bei der Ratte etwa $4 \mathrm{~cm} \mathrm{H}_{2} \mathrm{O}$ (Moreira et al. 1995), $6 \mathrm{~cm} \mathrm{H}_{2} \mathrm{O}$ beim Schwein (Protti et al. 2011) und $12 \mathrm{~cm} \mathrm{H}_{2} \mathrm{O}$ beim Menschen (Chiumello et al. 2008a). Daher variiert der, bei diesem strain auftretende stress und damit auch seine Grenzwerte erheblich zwischen den Spezies auf Grund von Unterschiedlichen spezifischen Elastancen, was beim Transfer von diesen Forschungsergebnissen zum Menschen bedacht werden muss. Der Einfluss der Spezies auf den schädlichen Leistungsgrenzwert ist folglich unklar und der Schwellenwert von $13 \frac{\mathrm{J}}{\mathrm{min}}$, welcher in diesem Experiment und vorherigen Experimenten gezeigt wurde (Cressoni et al. 2016), kann aber nicht direkt auf die Beatmungssituation beim Menschen übertragen werden (Collino et al. 2019). Es sei erwähnt, dass ein PEEP-Wert von 4 und $7 \mathrm{cmH}_{2} \mathrm{O}$ in Schweinen in etwa einem PEEP von 8 und $14 \mathrm{~cm} \mathrm{H}_{2} \mathrm{O}$ beim Menschen entspricht (Protti et al. 2015). Dies ist durch die Unterschiede in den spezifischen Lungenelastancen (ca. 6 vs $12 \mathrm{cmH}_{2} \mathrm{O}$ ) begründet (Protti et al. 2015). Im Zusammenhang mit dem Problem der Normierung wurde außerdem das Konzept der Intensität vorgeschlagen, sodass die Verteilung der mechanischen Leistung pro Lungenoberfläche berücksichtigt werden sollte, da eine $\mathrm{Zu}$ nahme der mechanischen Leistung bei gleichbleibender Oberfläche zu einer größeren Intensität und eine Vergrößerung der Lungenoberfläche (beispielsweise durch ein Rekrutierungsmanöver) zu einer kleineren Intensität führt (Samary et al. 2016). Dies wurde in diesem Experiment nicht weiter berücksichtigt und müsste für zukünftige Versuche näher untersucht werden.

Drittens wurde in diesem Experiment lediglich die mittlere mechanische Leistung untersucht. Dadurch ist nicht erklärt wie sich die mechanische Leistung örtlich, beispielsweise in der baby lung und ihrer Inhomogenität (Cressoni et al. 2014b) oder zeitlich, v.a. innerhalb der verschiedenen Phasen des Beatmungszyklus (Inspirations- und Exspirationsphase) verteilt (Energiedissipation) (Collino et al. 2019). Physikalisch betrachtet, wirkt bei der maschinellen Beatmung eine Kraft auf ein Material (Lungengewebe) und erzeugt interne Spannungen (stress), welche einer Verformung entgegenwirken (Pilkey 2004). Überschreiten diese Kräfte lokal oder global gewisse Schwellenwerte, kommt es zum Materialversagen (Pilkey 2004). In Bezug auf die Lungen wirkt dabei eine Kraft auf die extrazelluläre Matrix des Lungengewebes und verschiebt diese, wodurch sich strain und stress ergeben (Protti et al. 2015). Diese Volumenänderung (strain) bei einem bestimmten Druck (stress) ergibt als Produkt diejenige Energiemenge, welche auf die Lunge übertragen wird und möglicherweise also der Auslöser einer 
VILI im Sinne einer Materialermüdung darstellt (Protti et al. 2015). Lungen verhalten sich dabei wie viskoelastische Körper (Bayliss und Robertson 1939; Sugihara et al. 1972; Faffe und Zin 2009). Zum einen dissipieren diese Energie während der Inspiration und Exspiration, wodurch die während der Inspiration aufgebrachte Energie während der Exspiration nicht gänzlich abgegeben wird (Hysteresis). Die dabei dissipierte Energie ist die Fläche zwischen der Inspirations- und Exspirationskurve während einer Druck-Volumen-Schleife (Hysterese-Schleife) (Protti et al. 2016). Zum anderen zeigt sich ein intrinsisches Entspannen des Lungenparenchyms, da es bei einem konstanten strain zu Druckabfall kommt (stress relaxation) (Bayliss und Robertson 1939). Diese beiden Faktoren Hysterese und stress relaxation unterliegen allerdings auch noch weiteren Faktoren wie Gasfluss durch die distalen Atemwege, Oberflächenspannung und den mechanischen Eigenschaften des Thorax (Escolar and Escolar 2004). Der stress eines Objektes hängt von der Amplitude (strain) und Geschwindigkeit (strain rate) der Deformierung ab, weshalb zum Beispiel eine langsamere Deformation einen geringeren stress erzeugen und dadurch das Risiko eines Materialversagens verringert wird (Pilkey 2004). Normalerweise zeigen viskoelastische Polymere folglich eine höhere Anfälligkeit bei höheren strain rates (Graessley 2013). Eine hohe strain rate könnte also das Risiko einer VILI dadurch erhöhen, dass das viskoelastische Verhalten der Lungen zu einer ungünstigeren parenchymalen Energie-Dissipation der applizierten mechanischen Leistung führt (Protti et al. 2016). Die Wahl von strain und strain rate, welche eine möglichst geringe dynamische Hysterese und niedrige stress relaxation generieren, könnten demnach einer VILI vorbeugen (Protti et al. 2016). Daher liegt es nahe die bereits in der Berechnung der mechanischen Leistungsgleichung erfassten Parameter Beatmungsfrequenz und den inspiratorischen Fluss hinsichtlich ihres zeitlichen Verlaufs in zukünftigen Experimenten näher zu beleuchten.

Letztlich ist weiter unklar, ob dieselbe Gesamtleistung in unterschiedlicher Konstellation der Beatmungsparameter denselben Schaden hervorruft oder ob der Schaden von der Ausprägung der einzelnen Komponenten abhängig ist, wobei letzteres durch direkten Vergleich von ZEEP und PEEP von $4-7 \mathrm{cmH}_{2} \mathrm{O}$ durchaus möglich erscheint (Collino et al. 2019). In diesem Beispiel zeigt sich deutlich, dass trotz vergleichbarer mechanischer Gesamtleistungen ein eventuell auftretender Schaden sich signifikant unterscheiden kann (vgl. Abb. 5 und Abb. 18 und 19). Die Frage nach einer Gewichtung der einzelnen Parameter bleibt also weiterhin zu untersuchen. 


\subsection{Klinische Überlegungen zur Wahl des PEEP}

Welche Bedeutung könnten diese Erkenntnisse für die klinische Praxis haben? 1938 wurden die Effekte des PEEP als Ergänzung zur maschinellen Beatmung bei kardiogenen Lungenödem, Sepsis oder Asphyxie beschrieben (Barach et al. 1938), ehe 1946 die negativen Effekte auf die Hämodynamik quantifiziert wurden, wodurch der PEEP sehr zurückhaltend bis in die späten 1960er Jahre verwendet wurde (Gattinoni et al. 2017a). Dann nämlich wurde der PEEP bei Neonaten mit respiratrosichen Dystress-Syndrom ohne hämodynamische Konsequenzen angewendet (Gregory et al. 1971) und ähnliche Resultate in der Behandlung des damalig genannten adulten respiratorischen Dystress Syndrom (heute ARDS) erzielt (Ashbaugh et al. 1967). Die Entwicklung der Behandlung des ARDS und deren Pathologie verlief parallel zu der Entstehung der modernen Intensivmedizin, sodass der PEEP einen großen Anwendungsbereich fand (Gattinoni and Quintel 2016). Um Erkenntnisse unserer Versuche auf die Klinik anzuwenden, sei hervorgehoben, dass ein direkter Vergleich von ARDS-Lungen in Patienten und gesunden Lungen der Schweine durch die unterschiedlichen intrinsischen Lungeneigenschaften Einschränkungen unterliegt.

\subsubsection{Die Wahl des PEEP}

Dennoch lautet die zentrale Frage in der Patientenversorgung: Was ist der bestmögliche PEEP für den individuellen Patienten. In den Untersuchungen zur Bestimmung eines optimalen PEEP-Levels wurden diverse Kriterien wie Parameter der Lungenmechanik und des Sauerstofftransportes herangeführt und führten zu PEEP-Empfehlungen von 5 bis 25 $\mathrm{CmH}_{2} \mathrm{O}$ (Gattinoni et al. 2017a). Dabei sind zwei Dinge immer angeführt worden: Der positive Beitrag zur Oxygenierung und die hämodynamischen Nebenwirkungen. Die vielfältigen lokalen, aber auch systemischen Wirkungen und Nebenwirkungen des PEEP erfordern eine sorgfältig abgewogene individuelle PEEP-Einstellung nach physiologischen und pathophysiologischen atemmechanischen Eigenschaften des jeweiligen Patienten. Dabei sollten die möglichen Risiken bedacht werden und so der individuelle PEEP gewählt werden (Gattinoni et al. 2017a).

Beispielsweise wird in den deutschen Leitlinien empfohlen Patient nicht mit einem PEEP von unter $5 \mathrm{~cm} \mathrm{H}_{2} \mathrm{O} \mathrm{zu}$ beatmen (DGAI 2017). Es wurde eine Metaanalyse von insgesamt 575 lungengesunden Patienten (ohne ALI oder ARDS) durchgeführt, wobei festgestellt wurde, dass eine invasive Beatmung mit ZEEP (und einem Tidalvolumen von unter 8 
$\left.\frac{m l}{k g \text { Körpergewicht }}\right)$ das im Vergleich zu höheren Tidalvolumina und PEEP-Werten schlechtestes $\mathrm{PaO}_{2} / \mathrm{FiO}_{2}$-Verhältnis und niedrigsten Compliance hervorrief (Guo et al. 2016; DGAI 2017). Bei Patienten mit ARDS besteht eine Empfehlung durch die deutschen Leitlinien für einen höheren PEEP bei der invasiven Beatmung (DGAI 2017). Allerdings sollte bei vorgeschädigten Lungen weiter abgewogen werden: Beispielsweise konnte gezeigt werden, dass ein extern applizierter PEEP bei einer diffusen ARDS-Form, bei der alle Lungenareale gleichmäßig befallen sind, hauptsächlich zur alveolären Rekrutierung atelektatischer Lungenbereiche ohne wesentliche Überdehnung belüfteter Abschnitte führt, wohingegen bei regionalen ARDS-Formen dieses Verhältnis von Rekrutierung zu Überblähung schlechter ausfällt (DGAI 2017; Puybasset et al. 2000).

\subsubsection{Die Effekte des PEEP}

Letztlich richtet sich die Auswahl eines geeigneten PEEP zum einen nach dem Oxygenierungs-Vorteil und zum anderen nach den angeblichen lungenprotektiven Eigenschaften. Der positive Effekt auf die Oxygenierung wird dabei durch die Abnahme des intrapulmonalen Rechts-Links-Shunts durch offengehaltene Alveolen und Reduktion des intrathorakalen Drucks und somit des Herz-Zeit-Volumens hervorgerufen (Gattinoni et al. 2017a).

Der lungenprotektive Effekt des PEEP bezieht sich auf die Experimente von Webb und Tierney, bei denen während der zweistündigen Beatmung von Ratten eine vermehrte Bildung von Lungenödem vorgefunden wurde, wenn diese mit einem Plateaudruck von $45 \mathrm{Cm} \mathrm{H}_{2} \mathrm{O}$ bei einem PEEP von $0 \mathrm{cmH}_{2} \mathrm{O}$ im Vergleich zu einem Plataudruck von $45 \mathrm{cmH}_{2} \mathrm{O}$ und einem PEEP von $10 \mathrm{cmH}_{2} \mathrm{O}$ beatmet wurden (Webb and Tierney 1974) und gilt als Beweis des protektiven Effekts. Im gleichen Artikel wurde allerdings kein Schaden bei einem Plateaudruck von $30 \mathrm{~cm} \mathrm{H}_{2} \mathrm{O}$ bei einem PEEP von $0 \mathrm{cmH}_{2} \mathrm{O}$ vorgefunden (Gattinoni et al. 2017a; Webb and Tierney 1974). Die grundsätzliche Frage ist, ob der protektive Effekt des PEEP nur ein Nebeneffekt des verminderten Tidalvolumens, welches häufig mit einem applizierten PEEP verwendet wird, ist oder PEEP einen direkten Einfluss in die Verhinderung einer VILI hat. Zweifel an direkten lungenprotektiven Eigenschaften des PEEP (insbesondere bei höheren PEEP-Werten) kommen aus folgenden Gründen auf (Gattinoni et al. 2017a): 
Erstens ist der PEEP ein Druck und somit - wie durch unsere Experimente gezeigt - eine Komponente der auf das Lungenparenchym applizierten mechanischen Leistung (vgl. Abb. 5) (Collino et al. 2019). Um eine Lunge aufzublähen muss die aufgebrachte Energie größer sein als die erforderliche Energie um sie in dem ursprünglichen Öffnungszustand zu halten. Die dabei vorgedehnten Fasern können unphysiologische Dehnungsgrenzen überwinden und damit geschädigt werden (Gattinoni et al. 2016).

Zweitens legen vorherige Experimente im Zusammenhang mit der mechanischen Leistung nahe, dass beim Auftreten einer VILI lediglich eine überschrittene Leistungsgrenze, welche den PEEP einschließt, entscheidend ist und die Anwesenheit eines PEEP an sich irrelevant erscheint. Wenn also der PEEP so hoch ist, dass das assoziierte Tidalvolumen die physiologischen Grenzen der Lungenausdehnung erreicht, ist ein PEEP schädlich (Gattinoni et al. 2017a; Protti et al. 2015).

Drittens zeigt eine weitere Studie, trotz unterschiedlicher Herangehensweise, dass niedrige PEEP-Werte (im Bereich von $8 \mathrm{~cm} \mathrm{H}_{2} \mathrm{O}$ ) im Vergleich zu hohen PEEP-Werten (im Bereich von $15 \mathrm{CmH}_{2} \mathrm{O}$ ) keinen signifikanten Unterschied zeigten (Chiumello et al. 2014; Gattinoni et al. 2017a). Dies legt den Schluss nahe, dass ein Atelektrauma, welches angeblich mit niedrigeren PEEP-Werten assoziiert ist, einem Volutrauma, welches angeblich mit höheren PEEP-Werten assoziiert ist, im schädlichen Potenzial äquivalent erscheint (Gattinoni et al. 2017a). Dies soll nicht bedeuten, dass ein PEEP nicht nützlich sein kann, lediglich dass die Vorstellung eines protektiven PEEP konzeptionell möglicherweise einen falschen Ansatz darstellt (Gattinoni et al. 2017a). 


\subsection{Ausblick}

Aus den Limitationen dieses Versuchsaufbaus ergeben sich zahlreiche neue wissenschaftliche Fragestellungen: Welche Art der Normierung der mechanischen Leistung scheint angebracht? Wie ist die Leistung in Bezug auf Lungengröße, die Spezies und die zeitliche sowie örtliche Verteilung/Dissipation zu normieren? Wie verhält sich diese Problematik beim Transfer auf den Menschen? Inwieweit lässt sich das Konzept der Intensität näher untersuchen, sodass die Verteilung der mechanischen Leitung pro Lungenoberfläche berücksichtigt wird?

In Bezug auf die Formel für die mechanische Leistung stellen sich die folgenden Fragen: Führt eine identische Gesamtleistung in unterschiedlichen Konstellationen der Beatmungsparameter zu demselben Schaden? Wie verhält es sich mit einer Gewichtung der einzelnen Parameter und werden diese adäquat in der Leistungsgleichung widergespiegelt?

Eine zentrale Problematik stellt der Transfer der vorliegenden Ergebnisse auf den Menschen dar. Aktuelle retrospektive klinische Studien zeigen, dass die Überlegungen im Rahmen der mechanischen Leistung auch in die Klinik übertragbar scheinen (Serpa Neto et al. 2018). So konnte gezeigt werden, dass hohe mechanische Leistungen mit einer höheren intrahospitalen Mortalität und schlechterem Outcome während der Intensivbehandlung verbunden sind (Serpa Neto et al. 2018). Allerdings wurde in dem Zusammenhang wenig Rücksicht auf die Zusammensetzung der einzelnen Leistungskomponenten genommen, sodass eine separate Betrachtung - insbesondere im Hinblick auf den PEEP - notwendig wäre (Serpa Neto et al. 2018). So könnten zukünftige Studien auch diese und weitere Aspekte im klinischen Umfeld näher untersuchen. Die Betrachtungen dieser Arbeit beschränken sich auf gesunde Lungen. Wie verhält sich das Konzept der mechanischen Leistung auf (experimentell) vorgeschädigte Lungen und wie erfolgt eine Normierung in einem solchen Fall?

Wenn ein Grenzwert für die mechanische Leistung im Menschen identifiziert werden kann und die obengenannten Probleme gelöst werden könnten, könnte eine obere Schwelle definiert werden, welche bei der maschinellen Beatmung beim Patienten nicht überschritten werden sollte (Collino et al. 2019). Dadurch könnte ein objektivierbarer Indikator für die Notwendigkeit alternativer Methoden der respiratorischen Unterstützung gegeben sein (Collino et al. 2019). 


\subsection{Schlussfolgerung}

Aus den vorliegenden Ergebnissen lassen sich, unter Berücksichtigung der erläuterten Limitationen, folgende Thesen ableiten:

Ein herrschender ZEEP ist mit einem schweren Lungenschaden assoziiert.

PEEP-Werte von 4 und $7 \mathrm{CmH}_{2} \mathrm{O}$ reduzieren die dynamische Komponente der auf die Lunge wirkenden mechanischen Leistung und reduzieren den Lungenschaden.

Hohe PEEP-Werte $\left(11,14\right.$ und $\left.18 \mathrm{~cm} \mathrm{H}_{2} \mathrm{O}\right)$ erhöhen die mechanische Leistung allein durch die Erhöhung des PEEP-Wertes und führen zu schweren hämodynamischen und pulmonalen Schäden.

Ein Schwellenwert, ab dem Schäden über dem durchschnittlichen Schadensausmaß beobachtet wurden, lag bei etwa $13 \pm 1 \frac{\mathrm{J}}{\mathrm{min}}$.

Unter diesem Grenzwert der mechanischen Leistung kann ein applizierter PEEP die dynamische Komponente und dadurch das Auftreten von Atelektraumata reduzieren. Oberhalb dieser Grenze erhöht der PEEP durch die statische Komponente den auf die Lunge wirkenden Druck. Dadurch wird die Häufigkeit von potenziell tödlichen Lungenschäden und hämodynamische Störungen deutlich erhöht.

Dabei sei nochmals erwähnt, dass die untersuchten Parameter, deren Größenordnungen sowie davon ausgehende Berechnungen vielen Einschränkungen unterliegen. Zum einen beruhen diese nur auf einer Näherung des transpulmonalen Drucks und zum anderen wurden diese Werte tierexperimentell an beatmeten zuvor gesunden Schweinen erhoben. Daraus abgeleitet ergibt sich, dass diese Erkenntnisse noch nicht beim beatmeten Patienten überprüft und validiert wurden. Es resultieren daher deutliche Einschränkungen in der klinischen Anwendbarkeit und es sei auf die formulierten zukünftigen wissenschaftlichen Fragestellungen hingewiesen. 


\section{$5 \quad$ Zusammenfassung}

Bislang galt der PEEP im Zusammenhang mit VILI bei der maschinellen Beatmung als protektiv. Theoretische Überlegungen legen nahe, dass der PEEP im Konzept des Ergotraumas zur applizierten mechanischen Leistung beiträgt und somit womöglich ein schädigendes Potential aufweist. In dieser Dissertation konnte gezeigt werden, dass ein bei der maschinellen Beatmung applizierter PEEP als Komponente der mechanischen Leistung wahrscheinlich zu einem strukturellen Lungenschaden im Sinne eines Ergotraumas führt und entsprechende relevante Veränderungen der Lungenmechanik, Lungenstruktur, Hämodynamik und Organintegrität nach sich ziehen kann, sobald ein bestimmter Schwellenwert überschritten wird. Bei Schweinen entspricht dies etwa $12 \frac{\mathrm{J}}{\mathrm{min}}(0,2$ Watt $)$ über mehrere Stunden appliziert. Dazu wurden 40 weibliche Schweine über einen Zeitraum von 50 Stunden maschinell mit einem strain von 1,0 (Tidalvolumen $=$ FRC) beatmet. Im gewählten PEEP-Wert unterschieden sich die Versuchstiere mit PEEP-Werten von 0, 4, 7, 11, 14 und $18 \mathrm{~cm} \mathrm{H}_{2} 0$. In dieser Studie konnte dabei gezeigt werden, dass PEEP-Werte von 4 und $7 \mathrm{~cm} \mathrm{H}_{2} \mathrm{O}$ im Vergleich zu keinem PEEP die dynamische Komponente der mechanischen Leistung reduzieren können und dabei protektiv gegen Atelektrauma auftreten. Oberhalb einer definierten Schwelle für die mechanische Leistung, beispielsweise bei PEEP-Werten von 11, 14 und $18 \mathrm{~cm} \mathrm{H}_{2} 0$, waren die dynamischen Komponenten ähnlich, während die statischen PEEP-bedingten Komponenten stetig zunahmen. Durch solche höheren PEEP-Werte scheinen Volutrauma zu überwiegen und eine so induzierte Zerstörung manifestiert sich zunächst als schwere hämodynamische Beeinträchtigung, welche mit zunehmenden mechanischen und anatomischen Lungenschäden und letztlich einer erhöhten Mortalität einherging. Ein entsprechender Grenzwert für die mechanische Leistung, welche auf die Lungen wirkt, wurde mit etwa $13 \pm 1 \frac{\mathrm{J}}{\mathrm{min}}$ identifiziert. Dabei ist auch die Dauer der maschinellen Beatmung, welche im Normalfall bei einem Intensivpatienten länger als 24 Stunden beträgt, von hoher Bedeutung. Durch den PEEP erhöht sich die mechanische Leistung bei einer Langzeitbeatmung, wodurch der strain zunimmt und somit ein möglicherweise auftretender Lungenschaden verstärkt wird. Dieser Lungenschaden ist allerdings schwierig nachzuweisen. Mithilfe mehrerer moderner Methoden konnte gezeigt werden, dass der PEEP den Filtrationsdruck reduziert, wodurch die bestimmten Parameter wie das EVLW, das Lungengewicht und die W/D-Ratio abnehmen und sich diese dadurch nicht zur Bestimmung eines Lungenschadens eignen. Histopathologische Untersuchungen zeigten einen Lungenschaden oberhalb eines Grenzwertes der mechanischen Leistung, welcher lediglich vom PEEP verändert wurde, sodass wir zeigen konnten, dass ein PEEP per se einen Lungenschaden hervorrufen kann. 


\section{$6 \quad$ Anhang}

\section{Symbolübersicht}

$E_{L_{\text {spec }}}:$ Spezifische Lungenelastance

$P_{\text {Plateau }_{e s}}:$ Mittlerer Plateaudruck im Ösophagus

$P_{t p_{\text {insp }}}:$ Transpulmonaler Inspirationsdruck

$C_{O_{2 \text { art }}}: O_{2}$-Gehalt im arteriellen Blut

$C_{O_{2_{\text {cap }}}}: \mathrm{O}_{2}$-Gehalt im lungenkapillärem Blut

$C_{O_{2 m v}}: O_{2}$-Gehalt im gemischt-venösen Blut

$C_{L}:$ Compliance der Lunge

$C_{r s}:$ Compliance des respiratorischen Systems

$E_{L, \text { breath: }}$ Auf die Lunge wirkende Energie pro Atemzug

$E_{L}$ : Elastance der Lunge

$E_{r s, b r e a t h}:$ Auf das respiratorische System wirkende Energie pro Atemzug

$E_{r s}:$ Elastance des respiratorischen Systems

$E_{\text {tot }}:$ Elastance des gesamten Atemapparates

$E_{w}$ : Elastance der Brustwand

$F_{\text {Hook }}:$ Kraft im Hook'schen Gesetz

$k_{\text {Hook }}$ : Konstanten, welche die intrinsischen Elastizitätseigenschaften einer Feder beschreibt

$\mathrm{P}_{1}$ : Initialer Plateaudruck

$\mathrm{P}_{2}$ : Plateaudruck kurz vor der Exspiration

$P 1_{e s}$ : Initialer Plateaudruck im Ösophagus

$P 1_{L}$ : Initialer Plateaudruck in den Alveolen

$P 2_{e s}$ : Plateaudruck im Ösophagus kurz vor der Exspiration

$P 2_{L}$ : Druck in den Alveolen am Ende der Plateauphase

$P_{A W, \text { mean: }}$ Beatmungsmitteldruck 
$P_{D P, r s}:$ Treibende Druckdifferenz des respiratorischen Systems

$P_{D P, T P}:$ Transpulmonale treibende Druckdifferenz

$P_{\text {insp }}$ : Inspirationsdruck

$P_{\text {max }}, P_{\text {Peak }}:$ Spitzendruck

$P_{\text {Plateau: }}$ Plateaudruck

$P_{\text {pleu }}:$ Intrapleuraler Druck

$P_{r s}:$ Auf das respiratorische System wirkender Druck

$P_{t p_{e x p}}:$ Transpulmonaler Exspirationsdruck

PEE $P_{e s}:$ Druck im Ösophagus bei appliziertem PEEP

$P E E P_{t p}:$ Transpulmonaler positiver-end-exspiratorischen Druck

$\operatorname{Power}_{L_{\text {spec }}}$ : Auf die Lunge wirkende spezifische mechanische Leistung

Power $_{L}$ : Leistung, welche direkt auf die Lunge wirkt

Power $_{r s_{\text {spec }}}$ : Auf das respiratorische System wirkende spezifische mechanische Leistung

Power $_{r s}$ : Leistung, welche auf das respiratorische System wirkt

$R_{a w}:$ Resistance, Atemwegswiderstand

$R_{T}$ : Gewebswiderstand des Lungengewebes

RR: Respiratory rate (Atemfrequenz)

T: Dauer eines Atemzyklus

$\mathrm{T}_{\text {Exp }}$ : Exspirationszeit

$\mathrm{T}_{\text {Insp }}$ : Inspirationszeit

$V_{P E E P}:$ PEEP-bedingtes Volumen

$V$ : Volumen

$V_{\text {Plateau }}$ : stress-relaxation-bedingtes Volumen

$x_{\text {Hook }}$ : Länge im Hook'schen Gesetz

$Z E E P_{e s}:$ Druck im Ösophagus ohne applizierten PEEP 
$\Delta P_{a w_{P E E P}}:$ PEEP-Differenz in den Atemwegen

$\Delta P_{a w}:$ Atemwegs-Druckdifferenzen

$\Delta P_{\text {S }_{P E E P}}:$ PEEP-Differenz im Ösophagus

$\Delta P_{L, d y n}:$ Auf die Lunge wirkende dynamische Druckkomponente

$\triangle P_{L, P E E P}:$ Auf die Lunge wirkende PEEP-bedingte Druckkomponente

$\Delta P_{L, r e s}:$ Auf die Lunge wirkende resistive Durckkomponente

$\Delta P_{r s, d y n}:$ Auf das respiratorische System wirkende dynamische Druckkomponente

$\triangle P_{r S, P E E P}:$ Auf das respiratorische System wirkende PEEP-bedingte Druckkomponente

$\Delta P_{r s, r e s}:$ Auf das respiratorische System wirkende resistive Druckkomponente

$\Delta P_{t p}:$ Transpulmonale Druckveränderungen

$\Delta p:$ Druckdifferenz

$\Delta V / V_{T}$ : Volumenänderung durch das Tidalvolumen 


\section{$7 \quad$ Literaturverzeichnis}

Al-Jamal R, Ludwig MS (2001): Changes in proteoglycans and lung tissue mechanics during excessive mechanical ventilation in rats. Am J Physiol Lung Cell Mol Physiol 281, 10781087

Amato MB, Barbas CS, Medeiros DM, Magaldi RB, Schettino GP, Lorenzi-Filho G, Kairalla RA, Deheinzelin D, Munoz C, Oliveira R et al. (1998): Effect of a protective-ventilation strategy on mortality in the acute respiratory distress syndrome. N Engl J Med $\underline{338}$, $347-354$

Annat G, Viale JP, Bui Xuan B, Hadj Aissa O, Benzoni D, Vincent M, Gharib C, Motin J (1983): Effect of PEEP ventilation on renal function, plasma renin, aldosterone, neurophysins and urinary $\mathrm{ADH}$, and prostaglandins. Anesthesiology $\underline{58}, 136-141$

Ashbaugh DG, Bigelow DB, Petty TL, Levine BE (1967): Acute respiratory distress in adults. Lancet $\underline{2}, 319-323$

Barach AL, Martin J, Eckman M (1938): Positive pressure respiration and its application to the treatment of acute pulmonary edema. Ann Intern Med 12, 754

Bayliss LE, Robertson GW (1939): The visco-elastic properties of the lungs. Exp Physiol $\underline{29}, 27-47$

Beitler JR, Malhotra A, Thompson BT (2016): Ventilator-induced Lung Injury. Clin Chest Med $\underline{37}, 633-646$

Belperio JA, Keane MP, Burdick MD, Londhe V, Xue YY, Li K, Phillips RJ, Strieter RM (2002): Critical role for CXCR2 and CXCR2 ligands during the pathogenesis of ventilatorinduced lung injury. J Clin Invest 110, 1703-1716

Blankman P, Hasan D, Bikker IG, Gommers D (2016): Lung stress and strain calculations in mechanically ventilated patients in the intensive care unit. Acta Anaesthesiol Scand $\underline{60}$, 69-78

Briel M, Meade M, Mercat A, Brower RG, Talmor D, Walter SD, Slutsky AS, Pullenayegum E, Zhou Q, Cook D et al. (2010): Higher vs lower positive end-expiratory pressure in patients with acute lung injury and acute respiratory distress syndrome: systematic review and meta-analysis. JAMA $\underline{303}, 865-873$

Brower RG, Matthay MA, Morris A, Schoenfeld D, Thompson BT, Wheeler A (2000): Ventilation with lower tidal volumes as compared with traditional tidal volumes for acute lung injury and the acute respiratory distress syndrome. N Engl J Med $\underline{342}, 1301-1308$ Brower RG, Lanken PN, MacIntyre N, Matthay MA, Morris A, Ancukiewicz M, Schoenfeld D, Thompson BT (2004): Higher versus lower positive end-expiratory pressures in patients with the acute respiratory distress syndrome. N Engl J Med 351, 327-336 
Caironi P, Langer T, Carlesso E, Protti A, Gattinoni L (2011): Time to generate ventilatorinduced lung injury among mammals with healthy lungs: a unifying hypothesis. Intensive Care Med $\underline{37}, 1913-1920$

Cavalcante FSA, Ito S, Brewer K, Sakai H, Alencar AM, Almeida MP, Andrade JS, JR, Majumdar A, Ingenito EP, Suki B (2005): Mechanical interactions between collagen and proteoglycans: implications for the stability of lung tissue. J Appl Physiol (1985) 모, 672-679

Cavalcanti AB, Suzumura ÉA, Laranjeira LN, Paisani DdM, Damiani LP, Guimarães HP, Romano ER, Regenga MdM, Taniguchi LNT, Teixeira C et al. (2017): Effect of Lung Recruitment and Titrated Positive End-Expiratory Pressure (PEEP) vs Low PEEP on Mortality in Patients With Acute Respiratory Distress Syndrome: A Randomized Clinical Trial. JAMA $\underline{318}, 1335-1345$

Chiumello D, Carlesso E, Cadringher P, Caironi P, Valenza F, Polli F, Tallarini F, Cozzi P, Cressoni M, Colombo A et al. (2008a): Lung stress and strain during mechanical ventilation for acute respiratory distress syndrome. Am J Respir Crit Care Med 178, 346-355

Chiumello D, Cressoni M, Chierichetti M, Tallarini F, Botticelli M, Berto V, Mietto C, Gattinoni L (2008b): Nitrogen washout/washin, helium dilution and computed tomography in the assessment of end expiratory lung volume. Crit Care $\underline{12}$, 150-168

Chiumello D, Cressoni M, Carlesso E, Caspani ML, Marino A, Gallazzi E, Caironi P, Lazzerini M, Moerer O, Quintel M et al. (2014): Bedside selection of positive end-expiratory pressure in mild, moderate, and severe acute respiratory distress syndrome. Crit Care Med $\underline{42}, 252-264$

Chiumello D, Marino A, Brioni M, Cigada I, Menga F, Colombo A, Crimella F, Algieri I, Cressoni M, Carlesso E et al. (2016): Lung Recruitment Assessed by Respiratory Mechanics and Computed Tomography in Patients with Acute Respiratory Distress Syndrome. What Is the Relationship? Am J Respir Crit Care Med 193, 1254-1263

Colletti AA, Amini R, Kaczka DW (2011): Simulating ventilation distribution in heterogenous lung injury using a binary tree data structure. Comput Biol Med $\underline{41}$, 936-945

Collino F, Rapetti F, Vasques F, Maiolo G, Tonetti T, Romitti F, Niewenhuys J, Behnemann T, Camporota L, Hahn G et al. (2019): Positive End-expiratory Pressure and Mechanical Power. Anesthesiology 130, 119-130

Colmenero-Ruiz M, Fernandez-Mondejar E, Fernandez-Sacristan MA, Rivera-Fernandez R, Vazquez-Mata G (1997): PEEP and low tidal volume ventilation reduce lung water in porcine pulmonary edema. Am J Respir Crit Care Med 155, 964-970

Cressoni M, Chiumello D, Carlesso E, Chiurazzi C, Amini M, Brioni M, Cadringher P, Quintel M, Gattinoni L (2014a): Compressive forces and computed tomography-derived positive end-expiratory pressure in acute respiratory distress syndrome. Anesthesiology $\underline{121}, 572-581$ 
Cressoni M, Cadringher P, Chiurazzi C, Amini M, Gallazzi E, Marino A, Brioni M, Carlesso E, Chiumello D, Quintel M et al. (2014b): Lung inhomogeneity in patients with acute respiratory distress syndrome. Am J Respir Crit Care Med 189, 149-158

Cressoni M, Chiurazzi C, Gotti M, Amini M, Brioni M, Algieri I, Cammaroto A, Rovati C, Massari D, Di Castiglione CB et al. (2015): Lung inhomogeneities and time course of ventilator-induced mechanical injuries. Anesthesiology $\underline{123}$, 618-627

Cressoni M, Gotti M, Chiurazzi C, Massari D, Algieri I, Amini M, Cammaroto A, Brioni M, Montaruli C, Nikolla K et al. (2016): Mechanical Power and Development of Ventilatorinduced Lung Injury. Anesthesiology 124, 1100-1108

Cressoni M, Chiumello D, Algieri I, Brioni M, Chiurazzi C, Colombo A, Colombo A, Crimella F, Guanziroli M, Tomic I et al. (2017): Opening pressures and atelectrauma in acute respiratory distress syndrome. Intensive Care Med $\underline{43}$, 603-611

Crotti S, Mascheroni D, Caironi P, Pelosi P, Ronzoni G, Mondino M, Marini JJ, Gattinoni L (2001): Recruitment and derecruitment during acute respiratory failure: a clinical study. Am J Respir Crit Care Med 164, 131-140

DGAI (2017): S3-Leitlinie: Invasive Beatmung und Einsatz extrakorporaler Verfahren bei akuter respiratorischer Insuffizienz. https://www.awmf.org/uploads/tx_szleitlinien/001 0211_S3_Invasive_Beatmung_2017-12.pdf, abgerufen am: 15.11.2019

Dorinsky PM, Whitcomb ME (1983): The effect of PEEP on cardiac output. Chest $\underline{84}$, 210-216

Dreyfuss D, Soler P, Basset G, Saumon G (1988): High inflation pressure pulmonary edema. Respective effects of high airway pressure, high tidal volume, and positive end-expiratory pressure. Am Rev Respir Dis 137, 1159-1164

Dreyfuss D, Ricard J-D, Gaudry S (2015): Did studies on HFOV fail to improve ARDS survival because they did not decrease VILI? On the potential validity of a physiological concept enounced several decades ago. Intensive Care Med 41, 2076-2086

Escolar JD, Escolar A (2004): Lung hysteresis: a morphological view. Histol Histopathol $\underline{19}, 159-166$

Faffe DS, Zin WA (2009): Lung parenchymal mechanics in health and disease. Physiol Rev $\underline{89}, 759-775$

Ferguson ND, Cook DJ, Guyatt GH, Mehta S, Hand L, Austin P, Zhou Q, Matte A, Walter SD, Lamontagne F et al. (2013): High-frequency oscillation in early acute respiratory distress syndrome. N Engl J Med $\underline{368}, 795-805$

Fothergill J (1744): Observations on a case published in the last volume of the medical essays, \& of recovering a man dead in appearance, by distending the lungs with air. Phil Trans $\underline{43}, 275-281$ 
Frank JA, Wray CM, McAuley DF, Schwendener R, Matthay MA (2006): Alveolar macrophages contribute to alveolar barrier dysfunction in ventilator-induced lung injury. Am J Physiol Lung Cell Mol Physiol 291, 1191-1208

Frerichs I, Hinz J, Herrmann P, Weisser G, Hahn G, Dudykevych T, Quintel M, Hellige G (2002): Detection of local lung air content by electrical impedance tomography compared with electron beam CT. J Appl Physiol (1985) 93, 660-666

Froese AB, Bryan AC (1974): Effects of anesthesia and paralysis on diaphragmatic mechanics in man. Anesthesiology $\underline{41}, 242-255$

Fujita Y, Fujino Y, Uchiyama A, Mashimo T, Nishimura M (2007): High peak inspiratory flow can aggravate ventilator-induced lung injury in rabbits. Med Sci Monit 13, 95-100 Ganzert S, Möller K, Steinmann D, Schumann S, Guttmann J (2009): Pressure-dependent stress relaxation in acute respiratory distress syndrome and healthy lungs: An investigation based on a viscoelastic model. Crit Care $\underline{13}, 199-212$

Garcia CS, Abreu SC, Soares RM, Prota LF, Figueira RC, Morales MM, Capelozzi VL, Zin WA, Rocco PR (2008): Pulmonary morphofunctional effects of mechanical ventilation with high inspiratory air flow. Crit Care Med $\underline{36}$, 232-239

Gattinoni L (1993): Regional Effects and Mechanism of Positive End-Expiratory Pressure in Early Adult Respiratory Distress Syndrome. JAMA 269, 2122

Gattinoni L, Carlesso E, Cadringher P, Valenza F, Vagginelli F, Chiumello D (2003): Physical and biological triggers of ventilator-induced lung injury and its prevention. Eur Respir J Suppl 47, 15-25

Gattinoni L, Pesenti A (2005): The concept of "baby lung". Intensive Care Med $\underline{31}, 776-$ 784

Gattinoni L, Caironi P, Valenza F, Carlesso E (2006): The role of CT-scan studies for the diagnosis and therapy of acute respiratory distress syndrome. Clin Chest Med 27, 559-570

Gattinoni L, Quintel M (2016): Fifty Years of Research in ARDS Why Is Acute Respiratory Distress Syndrome So Important for Critical Care? Am J Respir Crit Care Med 194, 10511052

Gattinoni L, Tonetti T, Cressoni M, Cadringher P, Herrmann P, Moerer O, Protti A, Gotti M, Chiurazzi C, Carlesso E et al. (2016): Ventilator-related causes of lung injury: the mechanical power. Intensive Care Med 42, 1567-1575

Gattinoni L, Collino F, Maiolo G, Rapetti F, Romitti F, Tonetti T, Vasques F, Quintel M (2017a): Positive end-expiratory pressure: How to set it at the individual level. Ann Transl Med $\underline{5}, 288-301$ 
Gattinoni L, Marini JJ, Collino F, Maiolo G, Rapetti F, Tonetti T, Vasques F, Quintel M (2017b): The future of mechanical ventilation: lessons from the present and the past. Crit Care 21, 183-192

Ghadiali SN, Gaver DP (2008): Biomechanics of liquid-epithelium interactions in pulmonary airways. Respir Physiol Neurobiol 163, 232-243

Graessley WW: The Entanglement Concept in Polymer Rheology (Advances in Polymer Science); Springer-Verlag, Berlin 2013

Grasso S, Terragni P, Mascia L, Fanelli V, Quintel M, Herrmann P, Hedenstierna G, Slutsky AS, Ranieri VM (2004): Airway pressure-time curve profile (stress index) detects tidal recruitment/hyperinflation in experimental acute lung injury. Crit Care Med 32, 10181027

Gregory GA, Kitterman JA, Phibbs RH, Tooley WH, Hamilton WK (1971): Treatment of the idiopathic respiratory-distress syndrome with continuous positive airway pressure. $\mathrm{N}$ Engl J Med 284, 1333-1340

Guérin C, Papazian L, Reignier J, Ayzac L, Loundou A, Forel J-M (2016): Effect of driving pressure on mortality in ARDS patients during lung protective mechanical ventilation in two randomized controlled trials. Crit Care 20, 384-390

Guo L, Wang W, Zhao N, Guo L, Chi C, Hou W, Wu A, Tong H, Wang Y, Wang C et al. (2016): Mechanical ventilation strategies for intensive care unit patients without acute lung injury or acute respiratory distress syndrome: A systematic review and network meta-analysis. Crit Care 20, 226-238

Hahn G, Hartung C, Hellige G: Elektrische Impedanztomographie (EIT) als Methode zur regionalen Beurteilung der Lungenventilation: Entwicklung von Auswertverfahren zur Funktionserfassung und experimentelle Validierung; G. Fischer, Stuttgart 1998

Hotchkiss J, JR, Blanch L, Murias G, Adams AB, Olson DA, Wangensteen OD, Leo PH, Marini JJ (2000): Effects of decreased respiratory frequency on ventilator-induced lung injury. Am J Respir Crit Care Med 161, 463-468

Jiang D, Liang J, Fan J, Yu S, Chen S, Luo Y, Prestwich GD, Mascarenhas MM, Garg HG, Quinn DA et al. (2005): Regulation of lung injury and repair by Toll-like receptors and hyaluronan. Nat Med 11, 1173-1179

Kotani M, Kotani T, Li Z, Silbajoris R, Piantadosi CA, Huang YC (2004): Reduced inspiratory flow attenuates IL-8 release and MAPK activation of lung overstretch. Eur Respir J $\underline{24}, 238-246$

Kumar A, Pontoppidan H, Falke KJ, Wilson RS, Laver MB (1973): Pulmonary barotrauma during mechanical ventilation. Crit Care Med $1,181-186$ 
Larsen R, Ziegenfuß T: Beatmung: Indikationen - Techniken - Krankheitsbilder. 5. Auflage; Springer, Berlin 2013

Larsen R: Anästhesie. 11. Auflage; Urban \& Fischer Verlag, Philadelphia 2018

Lumb AB, Nunn JF: Nunn's applied respiratory physiology. 7. Auflage; Elsevier, London 2010

Maltais F, Reissmann H, Navalesi P, Hernandez P, Gursahaney A, Ranieri VM, Sovilj M, Gottfried SB (1994): Comparison of static and dynamic measurements of intrinsic PEEP in mechanically ventilated patients. Am J Respir Crit Care Med 150, 1318-1324

Marini JJ, Jaber S (2016): Dynamic predictors of VILI risk: beyond the driving pressure. Intensive Care Med 42, 1597-1600

Marini JJ (2016): Strain Rate and Cycling Frequency-The "Dynamic Duo" of Injurious Tidal Stress. Crit Care Med 44, 1800-1801

Meade MO, Cook DJ, Guyatt GH, Slutsky AS, Arabi YM, Cooper DJ, Davies AR, Hand LE, Zhou Q, Thabane L et al. (2008): Ventilation strategy using low tidal volumes, recruitment maneuvers, and high positive end-expiratory pressure for acute lung injury and acute respiratory distress syndrome: A randomized controlled trial. JAMA 299, 637-645

Mead J, Takishima T, Leith D (1970): Stress distribution in lungs: a model of pulmonary elasticity. J Appl Physiol 28, 596-608

Mercat A, Richard J-CM, Vielle B, Jaber S, Osman D, Diehl J-L, Lefrant J-Y, Prat G, Richecoeur J, Nieszkowska A et al. (2008): Positive end-expiratory pressure setting in adults with acute lung injury and acute respiratory distress syndrome: A randomized controlled trial. JAMA $\underline{299}$, 646-655

Mijailovich SM, Stamenovic D, Brown R, Leith DE, Fredberg JJ (1994): Dynamic moduli of rabbit lung tissue and pigeon ligamentum propatagiale undergoing uniaxial cyclic loading. J Appl Physiol (1985) 므, 773-782

Milic-Emili J (1989): Pulmonary flow resistance. Lung 167, 141-148

Moreira LF, Aires ST, Gobbi CF, Saldiva PH, Zin WA (1995): Respiratory system, lung, and chest wall mechanics after longitudinal laparotomy in rats. Eur Respir J $\underline{8}, 105-108$ Myers JC, Reilley TE, Cloutier CT (1988): Effect of positive end-expiratory pressure on extravascular lung water in porcine acute respiratory failure. Crit Care Med 16, 52-54

Oczenski W, Andel H, Werba A: Atmen, Atemhilfen: Atemphysiologie und Beatmungstechnik. 9. Auflage; Thieme, Stuttgart 2012

O'Neill LAJ (2005): TLRs play good cop, bad cop in the lung. Nat Med 11, 1161-1162 
Otis AB, McKerrow CB, Bartlett, Mead, McIlroy, N. J. Selverstone, E. P. Radford JR. (1956): Mechanical Factors in Distribution of Pulmonary Ventilation. J. Appl. Physiol. $\underline{8}$, $427-443$

Pape, H.-C., Klinke, R., Brenner, B., Silbernagl, S. (Hrsg.) (2018): Physiologie. 8. Auflage; Thieme, Stuttgart 2018

Parker JC, Hernandez LA, Peevy KJ (1993): Mechanisms of ventilator-induced lung injury. Crit Care Med 21, 131-143

Parker JC, Breen EC, West JB (1997): High vascular and airway pressures increase intersti-

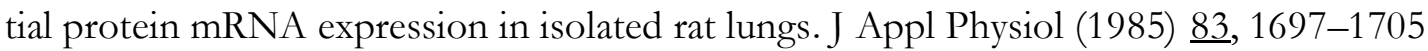

Pelosi P, D'Andrea L, Vitale G, Pesenti A, Gattinoni L (1994): Vertical gradient of regional lung inflation in adult respiratory distress syndrome. Am J Respir Crit Care Med $\underline{149}, 8-13$

Pelosi P, Cadringher P, Bottino N, Panigada M, Carrieri F, Riva E, Lissoni A, Gattinoni L (1999): Sigh in acute respiratory distress syndrome. Am J Respir Crit Care Med 159, 872 880

Pelosi P, Rocco PR (2008): Effects of mechanical ventilation on the extracellular matrix. Intensive Care Med $\underline{34}$, 631-639

Pilkey WD: Formulas for Stress, Strain, and Structural Matrices; John Wiley \& Sons, Hoboken 2004

Protti A, Cressoni M, Santini A, Langer T, Mietto C, Febres D, Chierichetti M, Coppola S, Conte G, Gatti S et al. (2011): Lung stress and strain during mechanical ventilation: any safe threshold? Am J Respir Crit Care Med 183, 1354-1362

Protti A, Andreis DT, Monti M, Santini A, Sparacino CC, Langer T, Votta E, Gatti S, Lombardi L, Leopardi O et al. (2013): Lung stress and strain during mechanical ventilation: any difference between statics and dynamics? Crit Care Med 41, 1046-1055

Protti A, Andreis DT, Milesi M, Iapichino GE, Monti M, Comini B, Pugni P, Melis V, Santini A, Dondossola D et al. (2015): Lung anatomy, energy load, and ventilator-induced lung injury. Intensive Care Med Exp $\underline{3}, 34-51$

Protti A, Maraffi T, Milesi M, Votta E, Santini A, Pugni P, Andreis DT, Nicosia F, Zannin E, Gatti S et al. (2016): Role of Strain Rate in the Pathogenesis of Ventilator-Induced Lung Edema. Crit Care Med 44, 838-45

Puybasset L, Gusman P, Muller JC, Cluzel P, Coriat P, Rouby JJ (2000): Regional distribution of gas and tissue in acute respiratory distress syndrome. III. Consequences for the effects of positive end-expiratory pressure. CT Scan ARDS Study Group. Adult Respiratory Distress Syndrome. Intensive Care Med 26, 1215-1227

Ranieri VM, Giunta F, Suter PM, Slutsky AS (2000): Mechanical ventilation as a mediator of multisystem organ failure in acute respiratory distress syndrome. JAMA $\underline{284}, 43-44$ 
Ranieri VM, Rubenfeld GD, Thompson BT, Ferguson ND, Caldwell E, Fan E, Camporota L, Slutsky AS (2012): Acute respiratory distress syndrome: the Berlin Definition. JAMA $\underline{307}, 2526-2533$

Rathgeber J: Grundlagen der maschinellen Beatmung: Einführung in die Beatmung für Ärzte und Pflegekräfte. 2. Auflage; Thieme, Stuttgart 2010

Retamal J, Borges JB, Bruhn A, Cao X, Feinstein R, Hedenstierna G, Johansson S, SuarezSipmann F, Larsson A (2016): High respiratory rate is associated with early reduction of lung edema clearance in an experimental model of ARDS. Acta Anaesthesiol Scand $\underline{60}, 79$ 92

Rich PB, Reickert CA, Sawada S, Awad SS, Lynch WR, Johnson KJ, Hirschl RB (2000): Effect of rate and inspiratory flow on ventilator-induced lung injury. J Trauma 49, 903-911

Rich PB, Douillet CD, Hurd H, Boucher RC (2003): Effect of ventilatory rate on airway cytokine levels and lung injury. J Surg Res $\underline{113}, 139-145$

Russell JA, Hoeffel J, Murray JF (1982): Effect of different levels of positive end-expiratory pressure on lung water content. J Appl Physiol (1985) 53, 9-15

Samary CS, Silva PL, Gama de Abreu M, Pelosi P, Rocco PRM (2016): Ventilator-induced Lung Injury: Power to the Mechanical Power. Anesthesiology 125, 1070-1071

Serpa Neto A, Deliberato RO, Johnson AEW, Bos LD, Amorim P, Pereira SM, Cazati DC, Cordioli RL, Correa TD, Pollard TJ et al. (2018): Mechanical power of ventilation is associated with mortality in critically ill patients: An analysis of patients in two observational cohorts. Intensive Care Med 44, 1914-1922

Sugihara T, Hildebrandt J, Martin CJ. (1972): Viscoelastic properties of alveolar wall. J Appl Physiol $\underline{33}$, 93-108

Suki B, Stamenovic D, Hubmayr R (2011): Lung parenchymal mechanics. Compr Physiol 1, 1317-1351

Suter PM, Fairley B, Isenberg MD (1975): Optimum end-expiratory airway pressure in patients with acute pulmonary failure. N Engl J Med 292, 284-289

Suttorp N, Möckel M, Siegmund B, Dietel M: Harrisons Innere Medizin. 19. Auflage; Thieme, New York 2016

Suzuki S, Hotchkiss JR, Takahashi T, Olson D, Adams AB, Marini JJ (2004): Effect of core body temperature on ventilator-induced lung injury. Crit Care Med $\underline{32}, 144-149$

Swan HJ, Ganz W, Forrester J, Marcus H, Diamond G, Chonette D (1970): Catheterization of the heart in man with use of a flow-directed balloon-tipped catheter. N Engl J Med 283 , $447-451$ 
Talmor D, Sarge T, Malhotra A, O'Donnell CR, Ritz R, Lisbon A, Novack V, Loring SH (2008): Mechanical ventilation guided by esophageal pressure in acute lung injury. N Engl J Med $\underline{359}$, 2095-2104

Taskar V, John J, Evander E, Robertson B, Jonson B (1997): Surfactant dysfunction makes lungs vulnerable to repetitive collapse and reexpansion. Am J Respir Crit Care Med $\underline{155}$, $313-320$

Tobin MJ (2000): Culmination of an era in research on the acute respiratory distress syndrome. N Engl J Med $\underline{342}, 1360-1361$

Tonetti T, Vasques F, Rapetti F, Maiolo G (2017): Driving pressure and mechanical power: new targets for VILI prevention. Ann Transl Med $\underline{5}$, 286-299

Toshima M, Ohtani Y, Ohtani O (2004): Three-dimensional architecture of elastin and collagen fiber networks in the human and rat lung. Arch Histol Cytol $\underline{67}, 31-40$

Tremblay L, Valenza F, Ribeiro SP, Li J, Slutsky AS (1997): Injurious ventilatory strategies increase cytokines and c-fos m-RNA expression in an isolated rat lung model. J Clin Invest 99, 944-952

Tschumperlin DJ, Oswari J, Margulies AS (2000): Deformation-induced injury of alveolar epithelial cells. Effect of frequency, duration, and amplitude. Am J Respir Crit Care Med $\underline{162}, 357-362$

Vlahakis NE, Schroeder MA, Pagano RE, Hubmayr RD (2001): Deformation-induced lipid trafficking in alveolar epithelial cells. Am J Physiol Lung Cell Mol Physiol 280, 938-946 Vlahakis NE, Schroeder MA, Pagano RE, Hubmayr RD (2002): Role of deformation-induced lipid trafficking in the prevention of plasma membrane stress failure. Am J Respir Crit Care Med 166, 1282-1289

Vlahakis NE, Hubmayr RD (2003): Response of alveolar cells to mechanical stress. Curr Opin Crit Care $\underline{9}, 2-8$

Vlahakis NE, Hubmayr RD (2005): Cellular stress failure in ventilator-injured lungs. Am J Respir Crit Care Med 171, 1328-1342

Webb HH, Tierney DF (1974): Experimental pulmonary edema due to intermittent positive pressure ventilation with high inflation pressures. Protection by positive end-expiratory pressure. Am Rev Respir Dis 110, 556-565

Wedler G: Lehrbuch der physikalischen Chemie. 4. Auflage; Wiley-VCH, Weinheim 1997 Ye H, Zhan Q, Ren Y, Liu X, Yang C, Wang C (2012): Cyclic deformation-induced injury and differentiation of rat alveolar epithelial type II cells. Respir Physiol Neurobiol $\underline{180}$, $237-246$

Yuan H, Ingenito EP, Suki B (1997): Dynamic properties of lung parenchyma: mechanical

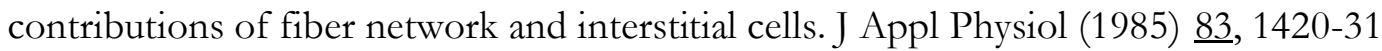




\section{Danksagung}

Hiermit möchte ich meiner Forschungsgruppe, in der diese Dissertationsschrift entstanden ist, sehr danken. Besonderer Dank gilt dabei Prof. Dr. med. Onnen Mörer für die Vergabe des Dissertationsthemas, die langjährige intensive Betreuung und die kritische Durchsicht dieser Dissertationsschrift. In den vielen motivierenden Diskussionen konnte ich nicht nur klinisch eine Menge lernen, sondern mich auch in wissenschaftlichen Aspekten weiterentwickeln.

Dank gilt auch Prof. Dr. med. Luciano Gattinoni, Prof. Dr. med. Micheal Quintel und Dr. med. Tommaso Tonetti, welche mir als Betreuer und Mentoren eine große Unterstützung während der Projektarbeit im tierexperiementellen OP, bei der Auswertung im Büro und bei der Ausarbeitung dieser Dissertation waren. 


\section{Lebenslauf}

Mein Name ist Tim Behnemann und ich wurde am 20.09.1993 in Buxtehude geboren. Ich besuchte in der Zeit von 2000 bis 2004 die Grundschule Scheeßel und anschließend die Eichenschule in Scheeßel bis zu meinem Abitur im Jahr 2012. Nach dem Abitur schloss ich eine Ausbildung zum Rettungssanitäter in Goslar im Jahr 2013 ab. Danach immatrikulierte ich mich im Fach Humanmedizin zum Sommersemester 2013 an der Georg-August-Universität in Göttingen. Im Oktober 2016 begann ich mit der Zusammenarbeit mit der Forschungsgruppe „Respiratorische Insuffizienz“ in Göttingen. Im Rahmen dieser Zusammenarbeit ist diese Dissertation entstanden. Zurzeit befinde ich mich im praktischen Jahr meines Medizinstudiums an der Universitätsmedizin Göttingen. 